The Astrophysical Journal Supplement Series, 90:433-465, 1994 January

(c) 1993. The American Astronomical Society. All rights reserved. Printed in U.S.A.

\title{
Ca II H AND K AND H $\alpha$ EMISSIONS IN CHROMOSPHERICALLY ACTIVE BINARY SYSTEMS (RS CANUM VENATICORUM AND BY DRACONIS)
}

\author{
M. J. Fernández-Figueroa, D. Montes, E. De Castro, and M. Cornide \\ Departamento de Astrofisica, Facultad de Físicas, Universidad Complutense de Madrid, 28040 Madrid, Spain \\ Received 1992 October 23; accepted 1993 June 21
}

\begin{abstract}
We report high-resolution observations of the $\mathrm{Ca}$ II $\mathrm{H}$ and $\mathrm{K}$ and Balmer $\mathrm{H} \alpha$ lines in 53 chromospherically active binary systems including the RS Canum Venaticorum binaries (RS CVn) and BY Draconis (BY Dra) classes. The rotational periods of the sample cover a range from 0.8 to 83.2 days. $\mathrm{H}$ and $\mathrm{K}$ emission-line surface fluxes have been calculated for all the stars in the sample. In spectra where the $\mathrm{H} \epsilon$ line appears in emission, absolute surface fluxes were also determined. The $\mathrm{Ca}$ II line profiles corresponding to different seasons and orbital phases are analyzed in order to determine the contribution of each component and to study the chromospheric activity variations. We have measured the $\mathrm{H} \alpha$ equivalent width of the program stars and the $\mathrm{H} \alpha$ core emission was determined by subtraction of the equivalent width of a presumably inactive star of the same spectral type and luminosity class. Three different types of $\mathrm{H} \alpha$ line profiles have been found: strong emission line, weak emission line with strong superposed absorption profile, and filled-in absorption line profile.
\end{abstract}

Subject headings: binaries: close - stars: chromospheres - stars: emission-line, $\mathrm{Be}$ - stars: late-type

\section{INTRODUCTION}

The RS Canum Venaticorum (RS CVn) binary systems, and BY Draconis (BY Dra) stars defined by Hall (1976) and Bopp \& Fekel (1977), respectively, are characterized by strong chromospheric activity, in every wavelength region in which they have been observed, and by the presence of a distortion of the light curve which migrates in phase with respect to the eclipses. The starspot model as explanation of the variability of these kind of systems is now widely accepted; i.e., the surface of the differentially rotating star exhibits dark and/or bright spots changing in size and brightness with time. Fekel, Moffett, \& Henry (1986), revised the original definition of both systems. For RS CVn systems in this new definition there is no restriction on the spectral type of the secondary star or on the period except for evolutionary constraints. The definition of BY Dra systems was expanded to include $F$ and $G$ dwarfs as well.

The general properties of RS CVn and BY Dra systems have been reviewed by Linsky (1984), Montesinos, Giménez, \& Fernández-Figueroa (1988), Strassmeier et al. (1988a), Dermican (1990, p. 431), Rodonò (1992), and Eker (1992). The strong chromospheric, transition region, and coronal activity in these systems are generally attributed to the deep convection zone and the fast rotation that drive the dynamo mechanism. The evolutionary status of these binary stars is not completely understood.

Enhanced emission cores in the $\mathrm{Ca}$ II resonance lines $\mathrm{H}$ and $\mathrm{K}$, and sometimes in the Balmer $\mathrm{H} \alpha$ line are the primary optical indicators of the chromospheric activity. However, in these kind of systems the $\mathrm{H}$ and $\mathrm{K}$ absolute fluxes and $\mathrm{H} \alpha$ equivalent width were rarely determined because of the complication in the analysis due to the binary nature of these stars. The reasons for this complication is that the emission of the active component is contaminated by the nonactive component which in many cases is the main contributor to the total observed flux. In addition, if both components are active their emissions can be blended.

Some recent spectroscopic studies of $\mathrm{Ca}$ II $\mathrm{H}$ and $\mathrm{K}$ and $\mathrm{H} \alpha$ lines in RS CVn and BY Dra systems have been presented by Bopp (1983, 1984), Fernández-Figueroa et al. (1986a, b, 1990), Fekel et al. (1986), Pasquini, Pallavicini, \& Pakull (1988), Strassmeier et al. (1990), De Castro et al. (1990), Armentia et al. (1990), Reglero et al. (1991), and Montes et al. (1993).

In this paper we report an analysis of the above mentioned activity indicators in a sample of 53 northern active binary systems selected from "A Catalog of Chromospherically Active Binary Stars" (Strassmeier et al. 1988b, hereafter CABS). In the selected sample we have also examined the behavior of the $\mathrm{Ca}$ II $\mathrm{H}$ and $\mathrm{K}$ emission. Firstly, the analyses of spectra at different orbital phases allowed us to establish which star is the source of the emission. Thus it was possible to find different cases, i.e., (a) The emission belongs to the cool component, as happens in the classical RS CVn systems; (b) the emission belongs only to the hot component as in the case of several BY Dra systems; or (c) the emission comes from both components. Secondly, we have measured the $\mathrm{Ca}$ II $\mathrm{H}$ and $\mathrm{K}$ emission-line absolute surface fluxes of these systems, many of which had not been previously determined.

Finally, we have also studied the behavior of the Balmer $\mathrm{H} \alpha$ line profile since the chromospheric activity level can be also inferred by the presence of emission or filled-in absorption in the core of the $\mathrm{H} \alpha$ line (Smith \& Bopp 1982; Barden 1985; Fekel et al. 1986; Bopp, Dempsey, \& Maniak 1988; Strassmeier et al. 1990).

In $\S 2$ we describe the instrumentation, date and spectral range of the observations. The reduction and spectral analysis, measurements of the emission fluxes and associated parame- 
ters are discussed in $\S 3$. The individual results are presented in $\S 4$ and in Figures 6 and 7 below. A final discussion of the global behavior of these systems is included in $\S 5$.

\section{OBSERVATIONS}

In Table 1 we show the adopted stellar parameters for the systems selected (CABS and references quoted therein). The parameter $S$ is the catalog number from CABS, the parameter $\delta m_{1}$ is from Giménez et al. (1991) and is defined as

$$
\delta m_{1}=m_{1}(\mathrm{~s})-m_{1}(\mathrm{o})
$$

where $m_{1}(\mathrm{~s})$ is the color index, $m_{1}$, obtained from the standard $m_{1}-(b-y)$ calibration for unevolved normal stars and $m_{1}(o)$ is the observed color index $m_{1}$. This parameter is currently used as an indicator of stellar metallicity.

The high-resolution spectroscopic observations ( $\mathrm{H} \alpha$ and $\mathrm{Ca}$ II $\mathrm{H}$ and $\mathrm{K}$ lines) presented here were obtained during several observing seasons from 1985 to 1991 and were carried out on two telescopes:

1. The $2.2 \mathrm{~m}$ telescope at the German Spanish Astronomical Observatory (CAHA) in Calar Alto (Almería, Spain), using the coudé spectrograph with the $\mathrm{f} / 3$ camera and a CCD as detector.

2. Isaac Newton Telescope (INT) at the Observatorio del Roque de los Muchachos (La Palma, Spain), using the IDS and IPCS or CCD as detectors.

The different observational seasons, the telescope used and the spectral region observed are given in Table 2 .

The reciprocal dispersion achieved with both instrumental configurations was $0.5 \AA$ pixel $^{-1}$ ( 1986 November) and $0.25 \AA$ pixel $^{-1}$ ( $\left.1989 \mathrm{July}\right)$ in the region of $\mathrm{H} \alpha$ line which yield spectral resolutions of 1.0 and $0.5 \AA$, respectively. In the region of $\mathrm{Ca}$ II lines the reciprocal dispersion achieved was $0.2 \AA \mathrm{pixel}^{-1}$ (1986 November) and $0.1 \AA$ pixel $^{-1}$ (in the other observational seasons) which yield spectral resolutions of 0.4 and 0.2 Å, respectively.

Many stars have been observed at different orbital phases and at different epochs when it was possible, which allowed us to find the possible phase variations and long-term changes of activity level indicating the presence of activity cycles. Following our paper on Strömgren photometry of active binaries (Giménez et al. 1991) the 53 stars of the sample are arranged in three groups according to their luminosity class.

Group 1 contains the systems whose active component is a main-sequence star (luminosity class V). When both components are on the main sequence, these stars belong to BY Dra systems, according to the definition of Fekel et al. (1986).

Group 2 includes the systems whose active component is an evolved star (luminosity class IV). Normally the companion star is also of luminosity class IV or V.

Finally, Group 3 contains systems whose active component is a giant or supergiant (luminosity classes III and II).

\section{DATA REDUCTION AND MEASURED PARAMETERS}

The wavelength-calibrated spectra have been extracted from the IPCS and CCD images, using the standard reduction procedures in the MIDAS package, and are corrected for atmospheric extinction by means of the semiempirical method by
Hayes \& Latham (1975). We have compared this method with the extinction curve from La Palma for INT spectra and also with the photometric extinction coefficients obtained for Calar Alto Observatory by Fabregat (1989), having found no significative deviations in any case.

The spectra in the region of $\mathrm{Ca} I \mathrm{H}$ and $\mathrm{K}$ have been calibrated using standard stars from the compilations of Oke \& Gunn (1983) and Barnes \& Hayes (1984). After the calibration the flux units are ergs $\mathrm{cm}^{-2} \mathrm{~s}^{-1} \AA^{-1}$. Night-to-night discrepancies found for the standard stars are within a $5 \%$. In the region of $\mathrm{H} \alpha$ line the spectra have been normalized to the flux of the continuum at $6540 \AA$.

\section{1. $\mathrm{Ca}$ II $\mathrm{H}$ and $\mathrm{K}$ Lines}

The emission fluxes of the $\mathrm{Ca}$ II $\mathrm{H}$ and $\mathrm{K}$ lines, $F_{\text {obs }}(\mathrm{H} ; \mathrm{K})$, were obtained by reconstruction of the absorption line profile below the emission peak(s), following the method given by Blanco et al. (1974). In this method the wing profiles are extrapolated smoothly toward the line center in order to define the upper photospheric level (pseudo-continuum). Although some authors (Linsky \& Ayres 1978) claim that the method of Blanco et al. overestimates the radiative photospheric contribution, we have adopted this method in all our papers where the chromospheric emission has been analyzed (FernándezFigueroa et al. 1986a, b; Armentia et al. 1990; Cornide et al. 1992) because $H 1$ and $K 1$ minima have essentially nonzero intensity in our spectra, where $\mathrm{H} 1$ and $\mathrm{K} 1$ refer to the minima outside the $\mathrm{H}$ and $\mathrm{K}$ line cores. Furthermore, this procedure can be the only useful for active binary systems since the emission usually originates at the cooler star of the system, whose contribution to the continuum in the region is very low. Therefore, an integration of the line from zero would generally take much of the flux coming from the hotter component.

For the cases in which there is only one emission component, the profile reconstruction is easily performed. However when the two components of the binary system are active the observed profile is the result of the emission from both components and to deblend the two contributions it is necessary to perform a Gaussian fit of the two emission lines and the absorption profile. In some of the examined systems the Balmer $\mathrm{H} \epsilon$ line $(3970.07 \AA)$ is presented as an emission feature. Since $\mathrm{H} \epsilon$ is very near to the Ca II H line (3968.47 $\AA$ ) it is also necessary to carry out another Gaussian fit to deblend these two emissions. The problem is still more complicated when the two components present $\mathrm{Ca}$ II $\mathrm{H}$ emission and also one or both components show $\mathrm{H} \epsilon$ in emission, in this case it is necessary to perform three or four Gaussian fits.

To convert the Earth-observed fluxes $\left[F_{\text {obs }}(H ; \mathrm{K})\right]$ into stellar surface flux $\left[F_{S}(\mathrm{H} ; \mathrm{K})\right]$ we have used the radii and distances, if available. In the case of stars whose radius is unknown, the linear relationship between the absolute surface flux at $3950 \AA$ (in ergs cm $\mathrm{cm}^{-2} \mathrm{~s}^{-1} \AA^{-1}$ ) and the color index $(V-$ $R$ ) of Pasquini et al. (1988) has been used.

Uncertainties in the measurement of the fluxes come from several sources. First, in the criterion to define the photospheric level of the absorption line (pseudocontinuum), the error is larger if the emission peak is centered at the same wavelength as that of the absorption line, since in this case, the reconstruction of the absorption profile is rather subjective. 
TABLE 1

Stellar Parameters (Groups 1, 2, AND 3)

\begin{tabular}{|c|c|c|c|c|c|c|c|c|c|}
\hline Name & $S$ & $T_{\mathrm{sp}}$ & SB & $\delta m 1$ & $V$ & $B-V$ & $V-R$ & $P_{\text {orb }}$ (days) & $P_{\text {rot }}$ (days) \\
\hline \multicolumn{10}{|c|}{ Group 1} \\
\hline 13 Cet .. & 5 & $\{\mathrm{~F} 7 \mathrm{~V} /\} \mathrm{G} 4 \mathrm{~V}$ & 1 & 0.01 & 5.20 & 0.56 & {$[0.48]$} & 2.0820 & \\
\hline DH Leo . & 73 & $\{\mathrm{~K} 0 \mathrm{~V} / \mathrm{K} 7 \mathrm{~V}\} / \mathrm{K} 5 \mathrm{~V}$ & 3 & 0.15 & 7.75 & 0.88 & 0.53 & 1.070354 & 1.0665 \\
\hline$\xi \mathrm{UMa}(\mathrm{B})$ & 76 & G5 V & 1 & 0.03 & 4.87 & 0.59 & {$[0.54]$} & 3.9805 & \\
\hline AS Dra & 84 & G4 V/G9 V & 2 & 0.09 & 8.00 & 0.73 & 0.60 & 5.414905 & 5.414905 \\
\hline HD $108102 \ldots \ldots$ & 85 & F8 V/F8 V & 2 & 0.02 & 8.16 & 0.52 & 0.40 & 0.9616 & 0.82 \\
\hline $\mathrm{TZ} \mathrm{CrB} \ldots \ldots \ldots$ & 107 & F6 V/G0 V & 2 & 0.03 & 5.70 & 0.47 & 0.50 & 1.1397912 & 1.1397912 \\
\hline V772 Her ........ & 119 & $\{\mathrm{G} 1 \mathrm{~V} /[\mathrm{K} 6 \mathrm{~V}]\} \mathrm{G} 5 \mathrm{~V}$ & 1 & & 7.07 & 0.59 & $\begin{array}{c}\{0.5 / \\
1.4\} 0.54\end{array}$ & 0.8794998 & 0.873 \\
\hline V815 Her.. & 122 & G5 V/[M1-2 V] & 1 & 0.10 & 7.76 & 0.72 & 0.54 & 1.8098368 & 1.8 \\
\hline BY Dra .......... & 126 & $\mathrm{~K} 4 \mathrm{~V} / \mathrm{K} 7.5 \mathrm{~V}$ & 2 & & 8.07 & 1.22 & 1.10 & 5.975112 & 3.827 \\
\hline V775 Her ......... & 129 & $\mathrm{~K} 0 \mathrm{~V} /[\mathrm{K} 5-\mathrm{M} 2 \mathrm{~V}]$ & 1 & 0.20 & 8.04 & 0.91 & 0.80 & 2.879395 & 2.898 \\
\hline V478 Lyr. & 131 & $\mathrm{G} 8 \mathrm{~V} /[\mathrm{dK}-\mathrm{dM}]$ & 1 & 0.08 & 7.72 & 0.74 & 0.65 & 2.130514 & 2.185 \\
\hline ER Vul . & 144 & G0 V/G5 V & 2 & & 7.27 & 0.68 & {$[0.50 / 0.64]$} & 0.69809510 & \\
\hline $\mathrm{KZ}$ And & 162 & $\mathrm{dK} 2 / \mathrm{dK} 2$ & 2 & 0.08 & 7.98 & 0.89 & {$[0.74 / 0.74]$} & 3.032867 & 3.03 \\
\hline \multicolumn{10}{|c|}{ Group 2} \\
\hline AR Psc . & 15 & /G8 IV & 2 & 0.19 & 7.28 & 0.87 & 0.69 & 14.300 & 12.245 \\
\hline LX Per. & 24 & G0 IV/K0 IV & 2 & 0.12 & 8.15 & 0.75 & $0.70 / 0.50$ & 8.038207 & 7.905 \\
\hline V711 Tau ........ & 27 & G5 IV/K1 IV & 2 & 0.28 & 5.70 & 0.92 & 0.77 & 2.83774 & 2.841 \\
\hline EI Eri $\ldots . . . \ldots \ldots$ & 31 & G5 IV & 1 & 0.08 & 6.95 & 0.67 & 0.61 & 1.94722 & 1.945 \\
\hline $54 \mathrm{Cam} . .$. & 61 & F9 IV/F9 IV & 2 & 0.03 & 6.52 & 0.62 & & 11.0764 & 10.163 \\
\hline UX Com ......... & 87 & G2/K1 [IV] & 2 & 0.20 & 10.0 & $0.61 / 1.05$ & {$[/ 0.75]$} & 3.642386 & 3.642386 \\
\hline RS CVn $\ldots \ldots \ldots$ & 89 & F5 IV/K0 IV & 2 & $0.05 / 0.31$ & 8.59 & 0.42 & 0.72 & 4.797851 & 4.7912 \\
\hline HR $5110 \ldots \ldots \ldots$ & 91 & F2 IV/K2 IV & 2 & 0.02 & 4.95 & 0.38 & 0.41 & 2.6131738 & $\ldots$ \\
\hline RV Lib .......... & 97 & G5 IV/K3 IV & 2 & & 9.00 & 1.04 & {$[0.61 / 0.89]$} & 10.722164 & 10.722164 \\
\hline SS Boo $\ldots$ & 98 & G0 V/K1 IV & 2 & 0.25 & 10.3 & $0.59 / 1.01$ & {$[0.50 / 0.75]$} & 7.606133 & 7.606133 \\
\hline RT CrB. & 103 & G0/G8 V-IV & 2 & 0.10 & 10.2 & 0.70 & & 5.1171590 & \\
\hline WW Dra ........ & 109 & G2 IV/K0 IV & 2 & 0.06 & 8.29 & 0.60 & {$[/ 0.72]$} & 4.6296166 & 4.6296166 \\
\hline HR $6469 \ldots \ldots$. & 113 & $\{\mathrm{~F} 2 \mathrm{~V} /[\mathrm{G} 0 \mathrm{~V}]\} \mathrm{G} 5 \mathrm{IV}$ & 1 & 0.04 & 5.51 & 0.68 & 0.49 & 2018. & 83.2 \\
\hline Z Her .... & 117 & F4 V-IV/K0 IV & 2 & 0.05 & 7.30 & 0.59 & {$[/ 0.69]$} & 3.9928012 & 3.962 \\
\hline MM Her ... & 118 & G2 IV/G8 IV & 2 & 0.05 & 9.51 & 0.84 & {$[/ 0.64]$} & 7.960322 & 7.936 \\
\hline AW Her ......... & 125 & G0/K1 IV & 2 & $0.14 / 0.40$ & 9.65 & {$[/ 0.99]$} & {$[/ 0.75]$} & 8.800760 & $\ldots$ \\
\hline 42 Cap .......... & 151 & G2 IV & 1 & 0.03 & 5.18 & 0.65 & & 13.1740 & \\
\hline RT Lac .......... & 153 & G9 IV/K1 IV & 2 & 0.39 & 8.84 & 1.14 & 0.77 & 5.074015 & 5.074015 \\
\hline $\mathrm{AR} \operatorname{Lac} . . \ldots \ldots \ldots$ & 155 & G2 IV/K0 IV & 2 & 0.10 & 6.09 & 0.72 & 0.77 & 1.98322195 & 1.98322195 \\
\hline SZ Psc .......... & 164 & F8 V/K1 IV & 2 & 0.23 & 7.20 & 0.84 & 0.70 & 3.9658663 & 3.955 \\
\hline \multicolumn{10}{|c|}{ Group 3} \\
\hline 33 Psc & 1 & KO III & 1 & & 4.61 & 1.04 & {$[0.77]$} & 72.93 & $\ldots$ \\
\hline$\zeta$ And & 7 & /K1 II & 1 & & 4.06 & 1.12 & 0.84 & 17.7692 & \\
\hline $12 \mathrm{Cam} . . . \ldots \ldots$ & 40 & K0 III & 1 & & 6.10 & 1.12 & {$[0.77]$} & 80.174469 & 80.94 \\
\hline CQ Aur ............... & 48 & $\mathrm{G} 2 / \mathrm{K} 0$ & 2 & $0.04 / 0.39$ & 9.00 & 0.87 & & 10.62148 & 10.56 \\
\hline$\sigma \mathrm{Gem} \ldots \ldots$ & 60 & K1 III & 1 & & 4.14 & 1.12 & 0.92 & 19.60458 & 19.410 \\
\hline RZ Cnc ... & 66 & K1 III/K3-4 III & 2 & & 8.69 & 1.18 & 0.81 & 21.643030 & 21.643030 \\
\hline 93 Leo .......... & 81 & A6 V:/G5 IV-III & 2 & & 4.50 & 0.55 & {$[0.18 / 0.64]$} & 71.6900 & 71.6900 \\
\hline DK Dra & 83 & K1 III/K1 III & 2 & & 6.14 & 1.12 & 0.89 & 64.44 & 63.75 \\
\hline GX Lib. & 100 & {$[\mathrm{G}-\mathrm{K}$ V]/K1 III } & 1 & & 7.31 & 1.02 & 0.84 & 11.1345 & 11.134 \\
\hline$\epsilon \mathrm{UMi} \ldots \ldots \ldots \ldots$ & 110 & A8-F0 V/G5 III & 1 & & 4.23 & 0.89 & {$[/ 0.89]$} & 39.4809 & \\
\hline V792 Her .. & 111 & F3 V/K0 III & 2 & & 8.50 & $0.45 / 1.07$ & {$[0.36 / 0.77]$} & 27.5384 & 27.07 \\
\hline DR Dra $\ldots \ldots \ldots$ & 115 & WD/K0-2 III & 1 & & 6.55 & 1.05 & {$[/ 0.81]$} & 39. & 31.5 \\
\hline o Dra ........... & 127 & G9 III & 1 & & 4.67 & 1.19 & {$[0.74]$} & 138.420 & 54.6 \\
\hline V1762 Cyg ....... & 132 & K1I V-III & 1 & & 5.99 & 1.09 & 0.81 & 28.59 & 27.8 \\
\hline HR 7428 & 136 & A0 V/K2 III-II & 1 & & 6.32 & 1.16 & & 108.5707 & \\
\hline V1764 Cyg....... & 137 & K1 III: & 1 & & 7.69 & 1.25 & 1.05 & 40.1425 & 39.878 \\
\hline HK Lac ......... & 154 & F1 V/K0 III & 1 & & 6.52 & 1.08 & 0.75 & 24.4284 & 24.4284 \\
\hline V350 Lac ........ & 157 & K2 III & 1 & & 6.38 & 1.17 & 0.84 & 17.755 & \\
\hline IM Peg .......... & 159 & K2 III-II & 1 & & 5.60 & 1.12 & 0.90 & 24.649 & 24.39 \\
\hline$\lambda$ And $\ldots \ldots \ldots \ldots$ & 166 & G8 IV-III & 1 & & 3.70 & 1.01 & 0.78 & 20.5212 & 53.952 \\
\hline
\end{tabular}


TABLE 2

SUMMARY OF OBSERVATIONS

\begin{tabular}{|c|c|c|c|}
\hline $\mathrm{O}$ & Date & Telescope & Observed lines \\
\hline & 1985 June & INT & $\mathrm{Ca}$ II $\mathrm{H}$ and $\mathrm{K}$ \\
\hline & 1986 November & CAHA & $\mathrm{Ca}$ II $\mathrm{H}$ and $\mathrm{K} \mathrm{H} \alpha$ \\
\hline & 1987 June & INT & $\mathrm{Ca}$ II $\mathrm{H}$ and $\mathrm{K}$ \\
\hline & 1988 January & CAHA & $\mathrm{Ca}$ II $\mathrm{H}$ and $\mathrm{K} \mathrm{H} \alpha$ \\
\hline $5 \ldots \ldots$ & 1988 July & INT & $\mathrm{Ca}$ II $\mathrm{H}$ and $\mathrm{K}$ \\
\hline $6 \ldots .$. & 1989 July & CAHA & $\mathrm{Ca}$ II $\mathrm{H}$ and $\mathrm{K} \mathrm{H} \alpha$ \\
\hline $7 \ldots \ldots$ & 1991 October & INT & $\mathrm{Ca}$ II $\mathrm{H}$ and $\mathrm{K}$ \\
\hline
\end{tabular}

Second, the measurements are more exact in the $\mathrm{K}$ line than in the $\mathrm{H}$ line because the absorption at $3968.47 \AA$ is more contaminated with $\mathrm{H} \epsilon$, while the $\mathrm{K}$ emission seems always to be free of blends.

In addition, the surface fluxes are affected by the accuracy of the factor $(R / d)^{2}$ or the Pasquini et al. (1988) calibration. It is difficult to estimate the total error in surface fluxes but a reasonable upper limit could be around $25 \%$.

In Tables 3, 4, and 5 we show the $\mathrm{Ca}$ II $\mathrm{H}$ and $\mathrm{K}$ emissionline fluxes for the groups 1,2 , and 3 , respectively $\left[F_{\text {obs }}(\mathrm{H} ; \mathrm{K})\right.$ and $F_{S}(\mathrm{H} ; \mathrm{K})$ in ergs $\mathrm{cm}^{-2} \mathrm{~s}^{-1}$ ]. Column (5) of Tables 3-5 gives the orbital phases $(\varphi)$ for each image; an asterisk $\left({ }^{*}\right)$ in this column denotes that these values are discussed in the text. In column (6), $\mathrm{H}$ and $\mathrm{C}$ mean emission belonging to Hot and Cool component, respectively, and $\mathrm{T}$ means that at these phases the emissions are blended, and only the total emission fluxes are given. Column (9) gives the parameter $C(\mathrm{~K})$ defined as

$$
C(\mathrm{~K})=\frac{F_{\mathrm{obs}}(\mathrm{K})}{F_{T}(\mathrm{~K})-F_{\mathrm{obs}}(\mathrm{K})},
$$

where $F_{T}(\mathrm{~K})$ is the total Ca II $\mathrm{K}$ emission line flux measured above the zero-flux level, whereas $F_{\text {obs }}(\mathrm{K})$ is the flux measured above the pseudocontinuum. This parameter indicates the relative flux of the emission above the absorption feature. $C(\mathrm{~K})$ is essentially the same as the criterion used by Wilson (1963), but in our case it is a measure, not an estimation.

The column heading with $\mathrm{O}$ lists a code for the date, telescope, and spectral range as explained in Table 2.

Several of these stars have been previously studied by us in $\mathrm{Ca}$ II $\mathrm{H}$ and $\mathrm{K}$ lines in preceding papers (whose references are given in the last column of Tables 3 and 4$)$.

The $\mathrm{H} \epsilon$ emission-lines fluxes $\left(F_{o b s}\right.$ and $\left.F_{\mathrm{S}}\right)$ are given in Table 6. $C(\mathrm{H} \epsilon)$ is defined for the $\mathrm{H} \epsilon$ emission line in the same way as $C(\mathrm{~K})$.

\subsection{Balmer $\mathrm{H} \alpha$ Line}

The $\mathrm{H} \alpha$ equivalent width $\left(\mathrm{EW}_{\alpha}\right.$ in Table 7) has been measured following the method of Bopp et al. (1988), which concentrates on the core of the line where chromospheric emission should be most visible. In this method the continuum is defined by a straight line from mean pixel values at 6540 and at $6590 \AA$ A. The integration limits to compute the equivalent width were obtained by extending the sides of the absorption profile in a straight line to the continuum level. The equivalent widths calculated by this method are given in Table 7, column (6). The plus sign is for an absorption line and the minus sign is for emission.

The emission equivalent width $\left(\mathrm{EW}_{e}\right.$ in Table 7$)$ for each program star was determined by subtracting the equivalent width of an inactive star of the same spectral type and luminosity class ( reference star) previously broadened with the appropriate value of $v \sin i$ of the program star.

Table 7 also contains for each program star the orbital phase $(\varphi)$ in column (4), the full width at half-maximum (FWHM) in column (5), the residual intensity $\left(R_{c}\right)$ defined as:

$$
R_{c}=1-\frac{I_{\text {line }}}{I_{\text {continuum }}}
$$

in column (7), and in the last column (O) a code for the date and telescope as explained in Table 2 is given. The spectral type, the measured equivalent width $\left(\mathrm{EW}_{\alpha}\right)$, and the residual intensity $\left(R_{c}\right)$ of each reference star are listed in Table 8.

\section{INDIVIDUAL RESULTS}

\subsection{Group 1 (Active Component of Luminosity Class V)}

\subsubsection{Ceti (HD 3196)}

13 Cet is a triple system (F7 V/)G4 V consisting of a shortperiod visual binary (ADS 490) in which the primary is a RS $\mathrm{CVn}$ single-lined spectroscopic binary. Several spectra in the $\mathrm{H}$ and $\mathrm{K}$ region of this system have been taken in 1986 November. A very faint emission, always centered at the absorption line, is observed. The F7 V component is the main contributor to the observed spectrum, hence should be the responsible of this emission.

The $\mathrm{H} \alpha$ line appears as a strong absorption.

\subsubsection{DH Leonis (HD 86590)}

This is a triple system (Barden 1984) whose primary and secondary components are active. Recently Strassmeier \& Fekel (1990) have classified this system as (K2 V/K5 V)K5 V.

In our observations at 0.32 phase $\mathrm{H}$ and $\mathrm{K}$ emissions from both components can be clearly seen. In addition a hump, likely due to $\mathrm{H} \epsilon$ emission, appears between the two $\mathrm{H}$ emissions. A Gaussian fit allowed us to separate these three lines (Fig. 1). At 0.55 phase the emissions of both components are blended, and using the same fitting method as described above we obtained $\mathrm{H}$ and $\mathrm{K}$ emissions from both components and $\mathrm{H} \epsilon$ emission (Fig. 1). At the two phases the hot component, to which the $\mathrm{H} \epsilon$ emission belongs, shows the strongest emission. The third component of the system is inactive.

\subsection{3. $\xi$ Ursae Majoris (B) ( 53 Ursae Majoris, HD 98230)}

It was possible to obtain spectra of both components of the visual pair (ADS 8119). The brighter component, $\xi \mathrm{UMa}(\mathrm{A})$, is an SB with an orbital period of 669.17 days. The Bright Star Catalogue (Hoffleit \& Jaschek 1982) assigns it a G0 V spectral type. It shows a very faint chromospheric emission. However, $\xi$ $\mathrm{UMa}(\mathrm{B})$, with a period of 3.98 days and a spectral type G5 V, shows stronger $\mathrm{H}$ and $\mathrm{K}$ emissions. In Table 3 only the data from the B component are given. 
TABLE 3

Ca II H AND K EMISSION-Line FluXes (Group 1)

\begin{tabular}{|c|c|c|c|c|c|c|c|c|c|c|c|c|}
\hline $\begin{array}{l}\text { Name } \\
\text { (1) }\end{array}$ & $\begin{array}{c}S \\
(2)\end{array}$ & $\begin{array}{c}R\left(R_{\odot}\right) \\
\quad(3)\end{array}$ & $\begin{array}{c}d(\mathrm{pc}) \\
(4)\end{array}$ & $\begin{array}{c}\varphi \\
(5)\end{array}$ & $\begin{array}{l}\text { Emission } \\
(6)\end{array}$ & $\begin{array}{c}F_{\text {obs }}(\mathrm{K}) \\
(7)\end{array}$ & $\begin{array}{c}F_{\text {obs }}(\mathrm{H}) \\
(8)\end{array}$ & $\begin{array}{c}\mathrm{C}(\mathrm{K}) \\
(9)\end{array}$ & $\begin{array}{c}F_{\mathrm{S}}(\mathrm{K}) \\
(10)\end{array}$ & $\begin{array}{c}F_{\mathrm{S}}(\mathrm{H}) \\
(11)\end{array}$ & $\begin{array}{c}\mathrm{O} \\
(12)\end{array}$ & $\begin{array}{l}\text { References } \\
\text { (13) }\end{array}$ \\
\hline 13 Cet & 5 & I & 17 & 0.17 & $\mathrm{C}$ & $1.91(-12)$ & $9.57(-13)$ & 0.16 & ${ }^{*} 1.00(+6)$ & $5.00(+4)$ & 2 & \\
\hline \multirow[t]{4}{*}{ DH Leo ........ } & 73 & $0.97 / 0.67$ & 32 & 0.32 & $\mathbf{H}$ & $1.26(-12)$ & $1.15(-12)$ & 1.14 & $2.69(+6)$ & $2.45(+6)$ & 4 & \\
\hline & & & & & $\mathrm{C}$ & $2.95(-13)$ & $3.06(-13)$ & 0.34 & $1.32(+6)$ & $1.37(+6)$ & 4 & \\
\hline & & & & 0.55 & $\mathbf{H}$ & $1.34(-12)$ & $1.29(-12)$ & 1.32 & $2.87(+6)$ & $2.76(+6)$ & 4 & \\
\hline & & & & & $\mathrm{C}$ & $4.07(-13)$ & $1.46(-13)$ & 0.40 & $1.82(+6)$ & $6.55(+5)$ & 4 & \\
\hline$\xi \operatorname{UMa}(B) \ldots \ldots$ & 76 & 1 & 8 & 0.49 & $\mathrm{C}$ & $6.82(-12)$ & $6.56(-13)$ & 0.62 & ${ }^{*} 1.97(+6)$ & $1.89(+6)$ & 4 & \\
\hline \multirow[t]{2}{*}{ AS Dra .......... } & 84 & / & 29 & 0.13 & $\mathbf{H}$ & $3.35(-13)$ & $5.14(-13)$ & & $5.35(+6)$ & $8.20(+6)$ & 1 & 1 \\
\hline & & & & & C & $2.85(-13)$ & $1.36(-13)$ & & $7.45(+6)$ & $3.56(+6)$ & 1 & 1 \\
\hline \multirow[t]{4}{*}{ HD $108102 \ldots$} & 85 & $1.1 / 1.1$ & 86 & $*$ & 1 & $3.46(-13)$ & $2.81(-13)$ & 0.21 & $4.15(+6)$ & $3.37(+6)$ & 4 & \\
\hline & & & & & 2 & $2.60(-13)$ & $1.67(-13)$ & 0.18 & $3.12(+6)$ & $2.01(+6)$ & 4 & \\
\hline & & & & $*$ & 1 & $3.39(-13)$ & $2.16(-13)$ & 0.24 & $4.07(+6)$ & $2.60(+6)$ & 4 & \\
\hline & & & & & 2 & $2.77(-13)$ & $1.40(-13)$ & 0.19 & $3.33(+6)$ & $1.68(+6)$ & 4 & \\
\hline \multirow[t]{3}{*}{ TZ CrB ........ } & 107 & $1.22 / 1.21$ & 21 & 0.77 & 1 & $8.80(-12)$ & $7.85(-12)$ & 0.34 & $5.21(+6)$ & $4.65(+6)$ & 4 & \\
\hline & & & & & 2 & $7.55(-12)$ & $7.68(-12)$ & 0.35 & $4.47(+6)$ & $4.55(+6)$ & 4 & \\
\hline & & & & 0.54 & $\mathrm{~T}$ & $1.38(-11)$ & $1.22(-11)$ & 0.54 & $8.17(+6)$ & $7.22(+6)$ & 6 & \\
\hline \multirow[t]{4}{*}{ V772 Her...... } & 119 & $(0.90 / 0.58)$ & 32 & 0.86 & $\mathrm{H}$ & $7.30(-13)$ & $4.80(-13)$ & 0.49 & $1.77(+6)$ & $1.17(+6)$ & 6 & 2 \\
\hline & & 0.91 & & & $\mathrm{C}$ & $2.70(-13)$ & $2.50(-13)$ & & $6.70(+5)$ & $6.20(+5)$ & 6 & 2 \\
\hline & & & & 0.28 & $\mathrm{H}$ & $6.90(-13)$ & $3.60(-13)$ & 0.40 & $1.68(+6)$ & $8.80(+5)$ & 6 & 2 \\
\hline & & & & & $\mathrm{C}$ & $2.60(-13)$ & $2.60(-13)$ & & $6.50(+5)$ & $6.50(+5)$ & 6 & 2 \\
\hline V815 Her. & 122 & 0.93:/ & 31 & 0.68 & $\mathrm{H}$ & $1.70(-12)$ & $1.28(-12)$ & 1.33 & $3.71(+6)$ & $2.79(+6)$ & 5 & \\
\hline \multirow[t]{8}{*}{ BY Dra ......... } & 126 & $1.2 /$ & 16 & 0.71 & $\mathrm{H}$ & $1.45(-12)$ & $1.22(-12)$ & 4.11 & $4.82(+5)$ & $4.18(+5)$ & 5 & \\
\hline & & & & & $\mathrm{C}$ & $6.35(-13)$ & $4.89(-13)$ & 1.93 & & & 5 & \\
\hline & & & & 0.73 & $\mathrm{H}$ & $1.43(-12)$ & $1.34(-12)$ & 3.50 & $4.76(+5)$ & $3.32(+5)$ & 5 & \\
\hline & & & & & $\mathrm{C}$ & $7.78(-13)$ & $5.32(-13)$ & 2.01 & & & 5 & \\
\hline & & & & 0.22 & $\mathrm{H}$ & $1.56(-12)$ & $1.38(-12)$ & 3.32 & $5.19(+5)$ & $4.60(+5)$ & 5 & \\
\hline & & & & & $\mathrm{C}$ & $9.93(-13)$ & $8.74(-13)$ & 1.73 & & & 5 & \\
\hline & & & & 0.23 & $\mathrm{H}$ & $1.62(-12)$ & $1.22(-12)$ & 3.03 & $5.38(+5)$ & $4.04(+5)$ & 5 & \\
\hline & & & & & $\mathrm{C}$ & $9.32(-13)$ & $8.35(-13)$ & 1.85 & & & 5 & \\
\hline \multirow[t]{5}{*}{ V775 Her ...... } & 129 & $0.85 /$ & 24 & 0.04 & $\mathrm{H}$ & $1.95(-12)$ & $1.54(-12)$ & 3.43 & $3.05(+6)$ & $2.41(+6)$ & 5 & \\
\hline & & & & 0.06 & $\mathrm{H}$ & $2.03(-12)$ & $1.73(-12)$ & 4.54 & $3.18(+6)$ & $2.70(+6)$ & 5 & \\
\hline & & & & 0.37 & $\mathrm{H}$ & $1.83(-12)$ & $1.58(-12)$ & 3.82 & $2.87(+6)$ & $2.47(+6)$ & 5 & \\
\hline & & & & 0.40 & $\mathrm{H}$ & $1.88(-12)$ & $1.74(-12)$ & 3.72 & $2.94(+6)$ & $2.73(+6)$ & 5 & \\
\hline & & & & 0.07 & $\mathrm{H}$ & $1.90(-12)$ & $1.31(-12)$ & 2.70 & $2.97(+6)$ & $2.05(+6)$ & 5 & \\
\hline \multirow[t]{3}{*}{ V478 Lyr ...... } & 131 & $\geq 0.9 /$ & 26 & 0.40 & $\mathrm{H}$ & $1.76(-12)$ & $1.52(-12)$ & 1.79 & *2.89(+6) & $2.49(+6)$ & 5 & \\
\hline & & & & 0.85 & $\mathrm{H}$ & $1.54(-12)$ & $1.35(-12)$ & 1.90 & $* 2.53(+6)$ & $2.21(+6)$ & 5 & \\
\hline & & & & 0.77 & $\mathrm{H}$ & $1.31(-12)$ & $1.20(-12)$ & 1.43 & *2.14 (+6) & $1.97(+6)$ & 5 & \\
\hline \multirow[t]{14}{*}{ ER Vul ........ } & 144 & $1.23 / 1.23$ & 45 & 0.04 & $\mathrm{H}$ & $3.63(-13)$ & $3.35(-13)$ & 0.24 & $9.55(+5)$ & $8.81(+5)$ & 5 & 3 \\
\hline & & & & & $\mathrm{C}$ & $6.17(-13)$ & & & $1.62(+6)$ & & 5 & 3 \\
\hline & & & & 0.10 & $\mathrm{H}$ & $8.20(-13)$ & $1.08(-13)$ & 0.13 & $2.16(+6)$ & $2.84(+5)$ & 5 & 3 \\
\hline & & & & & $\mathrm{C}$ & $1.36(-12)$ & $5.30(-13)$ & & $3.59(+6)$ & $1.39(+6)$ & 5 & 3 \\
\hline & & & & 0.92 & $\mathrm{H}$ & $6.12(-13)$ & & 0.39 & $1.61(+6)$ & & 5 & 3 \\
\hline & & & & & $\mathrm{C}$ & $3.66(-13)$ & $7.00(-14)$ & & $9.63(+5)$ & $1.84(+5)$ & 5 & 3 \\
\hline & & & & 0.00 & $\mathrm{H}$ & $6.20(-13)$ & & & $1.63(+6)$ & & 5 & 3 \\
\hline & & & & & $\mathrm{C}$ & $1.15(-12)$ & & & $3.02(+6)$ & & 5 & 3 \\
\hline & & & & 0.87 & $\mathrm{H}$ & $8.93(-13)$ & $5.62(-13)$ & 0.13 & $2.35(+6)$ & $1.48(+6)$ & 6 & 3 \\
\hline & & & & & $\mathrm{C}$ & $5.19(-13)$ & $4.26(-13)$ & & $1.37(+6)$ & $1.12(+6)$ & 6 & 3 \\
\hline & & & & 0.24 & $\mathrm{H}$ & $9.04(-13)$ & $8.78(-13)$ & 0.18 & $2.38(+6)$ & $2.31(+6)$ & 6 & 3 \\
\hline & & & & & $\mathrm{C}$ & $9.66(-13)$ & $1.29(-12)$ & & $2.54(+6)$ & $3.39(+6)$ & 6 & 3 \\
\hline & & & & 0.71 & $\mathbf{H}$ & $8.11(-13)$ & $7.22(-13)$ & 0.18 & $2.13(+6)$ & $1.90(+6)$ & 6 & 3 \\
\hline & & & & & $\mathrm{C}$ & $1.13(-12)$ & $8.09(-13)$ & & $2.97(+6)$ & $2.13(+6)$ & 6 & 3 \\
\hline \multirow[t]{2}{*}{$\mathrm{KZ}$ And $\ldots \ldots \ldots$} & 162 & $\geq 0.74$ & 23 & 0.33 & $\mathbf{P}$ & $6.35(-13)$ & $5.76(-13)$ & 1.34 & $* 1.21(+6)$ & $1.09(+6)$ & 6 & \\
\hline & & & & & $\mathrm{S}$ & $6.01(-13)$ & $7.09(-13)$ & 1.31 & ${ }^{*} 1.14(+6)$ & $1.35(+6)$ & 6 & \\
\hline
\end{tabular}

REFERENCES._-(1) Fernández-Figueroa et al. 1986a; (2) Reglero et al. 1991; (3) Fernández-Figueroa et al. 1990.

\subsubsection{HD 108102}

Fernández-Figueroa et al. (1986a) reported $\mathrm{H}$ and $\mathrm{K}$ emissions arising from both components. New observations confirm this behavior (Fig. 2). Because the computed phases obtained by means of the ephemeris tabulated in CABS do not agree with the observed spectra, we cannot establish which star is responsible for each emission. Table 3 contains the emission fluxes, of blue- and redshifted lines, computed by fitting Gaussian profiles to the observed spectrum.

\subsection{5. $\sigma^{2}$ Coronae Borealis (TZ Coronae Borealis, HD 146631)}

This is a double-lined and double-emission spectroscopic binary. Previous observations carried out by us (FernándezFigueroa et al. 1986a) showed that the secondary component 
TABLE 4

Ca II H AND K EMISSION-LINE FluXES (GROUP 2)

\begin{tabular}{|c|c|c|c|c|c|c|c|c|c|c|c|c|}
\hline $\begin{array}{l}\text { Name } \\
\text { (1) }\end{array}$ & $\begin{array}{c}S \\
(2)\end{array}$ & $\begin{array}{c}R\left(R_{\odot}\right) \\
\quad(3)\end{array}$ & $\begin{array}{c}d(\mathrm{pc}) \\
(4)\end{array}$ & $\begin{array}{c}\varphi \\
(5)\end{array}$ & $\begin{array}{c}\text { Emission } \\
(6)\end{array}$ & $\begin{array}{c}F_{\text {obs }}(\mathrm{K}) \\
(7)\end{array}$ & $\begin{array}{c}F_{\text {obs }}(\mathrm{H}) \\
(8)\end{array}$ & $\begin{array}{c}\mathrm{C}(\mathrm{K}) \\
(9)\end{array}$ & $\begin{array}{c}F_{\mathrm{S}}(\mathrm{K}) \\
(10)\end{array}$ & $\begin{array}{c}F_{\mathrm{S}}(\mathrm{H}) \\
(11)\end{array}$ & $\begin{array}{c}\mathrm{O} \\
(12)\end{array}$ & $\begin{array}{l}\text { References } \\
\text { (13) }\end{array}$ \\
\hline \multirow[t]{3}{*}{ AR Psc $\ldots \ldots \ldots$} & 15 & $\geq 1.5$ & 70 & 0.33 & $\mathrm{C}$ & $7.25(-13)$ & $5.96(-13)$ & 1.97 & *3.17 (+6) & $2.61(+6)$ & 2 & 1 \\
\hline & & & & 0.39 & $\mathrm{C}$ & $7.05(-13)$ & $6.47(-13)$ & 1.91 & $* 3.08(+6)$ & $2.83(+6)$ & 2 & 1 \\
\hline & & & & 0.67 & $\mathrm{C}$ & $7.35(-13)$ & $6.11(-13)$ & 1.64 & $* 3.21(+6)$ & $2.67(+6)$ & 2 & 1 \\
\hline \multirow{4}{*}{ LX Per......... } & 24 & $1.6 / 3.16$ & 145 & 0.86 & $\mathrm{C}$ & $5.98(-13)$ & $5.49(-13)$ & & $2.48(+6)$ & $2.27(+6)$ & 1 & 2 \\
\hline & & & & 0.99 & $\mathrm{C}$ & $5.18(-13)$ & $5.71(-13)$ & & $2.14(+6)$ & $2.36(+6)$ & 1 & 2 \\
\hline & & & & 0.12 & $\mathrm{C}$ & $6.24(-13)$ & $5.32(-13)$ & & $2.58(+6)$ & $2.20(+6)$ & 1 & 2 \\
\hline & & & & 0.86 & $\mathrm{C}$ & $5.76(-13)$ & $4.94(-13)$ & & $2.38(+6)$ & $2.04(+6)$ & 1 & 2 \\
\hline \multirow[t]{5}{*}{ V711 Tau ...... } & 27 & $1.3 / 3.9$ & 36 & 0.16 & $\mathrm{H}$ & $5.89(-12)$ & $4.85(-12)$ & 0.40 & $8.88(+6)$ & $7.31(+6)$ & 2 & \\
\hline & & & & & $\mathrm{C}$ & $4.61(-11)$ & $4.13(-11)$ & 1.76 & $7.72(+6)$ & $6.93(+6)$ & 2 & \\
\hline & & & & 0.57 & $\mathrm{~T}$ & $5.17(-11)$ & $4.69(-11)$ & 1.56 & $8.67(+6)$ & $7.86(+6)$ & 2 & \\
\hline & & & & 0.46 & $\mathrm{~T}$ & $4.14(-11)$ & $3.97(-11)$ & 1.74 & $6.94(+6)$ & $6.66(+6)$ & 4 & \\
\hline & & & & 0.83 & $\mathrm{~T}$ & $3.89(-11)$ & $2.52(-11)$ & 1.45 & $6.51(+6)$ & $4.23(+6)$ & 4 & \\
\hline EI Eri & 31 & $\geq 1.9 /$ & 75 & 0.94 & $\mathrm{~T}$ & $7.14(-13)$ & $5.12(-13)$ & 0.88 & $* 3.80(+6)$ & $2.72(+6)$ & 4 & \\
\hline \multirow[t]{4}{*}{54 Cam ......... } & 61 & $3.14 / 2.64$ & 38 & 0.73 & $\mathrm{C}$ & $2.52(-12)$ & $2.07(-12)$ & 0.35 & $1.03(+6)$ & $8.40(+5)$ & 2 & \\
\hline & & & & 0.10 & $\mathrm{C}$ & $2.76(-12)$ & $2.55(-12)$ & 0.36 & $1.12(+6)$ & $1.04(+6)$ & 2 & \\
\hline & & & & 0.18 & $\mathrm{C}$ & $4.08(-12)$ & $3.56(-12)$ & 0.53 & $1.66(+6)$ & $1.45(+6)$ & 2 & \\
\hline & & & & 0.16 & $\mathrm{C}$ & $3.77(-12)$ & $3.93(-12)$ & 0.62 & $1.54(+6)$ & $1.60(+6)$ & 4 & \\
\hline \multirow{2}{*}{ UX Com ........ } & 87 & $1.0 / 2.5$ & 350 & 0.47 & $\mathrm{C}$ & $2.51(-13)$ & $2.73(-13)$ & & $9.63(+6)$ & $1.05(+7)$ & 1 & 3 \\
\hline & & & & 0.73 & $\mathrm{C}$ & $3.43(-13)$ & $3.13(-13)$ & & $1.32(+7)$ & $1.21(+7)$ & 1 & 3 \\
\hline \multirow{2}{*}{ RS CVn ........ } & 89 & $1.88 / 4.10$ & 145 & 0.86 & $\mathrm{C}$ & $8.20(-13)$ & $6.54(-13)$ & 0.56 & $2.02(+6)$ & $1.61(+6)$ & 4 & \\
\hline & & & & 0.69 & $\mathrm{C}$ & $9.10(-13)$ & $5.34(-13)$ & 0.60 & $2.24(+6)$ & $1.31(+6)$ & 4 & \\
\hline \multirow[t]{5}{*}{ HR $5110 \ldots \ldots$} & 91 & $3.10 / 2.85$ & 53 & 0.19 & $\mathrm{C}$ & $2.51(-12)$ & $2.46(-12)$ & 0.16 & $1.70(+6)$ & $1.67(+6)$ & 4 & \\
\hline & & & & 0.58 & $\mathrm{C}$ & $2.04(-12)$ & $1.58(-12)$ & 0.11 & $1.39(+6)$ & $1.08(+6)$ & 4 & \\
\hline & & & & 0.34 & $\mathrm{C}$ & $2.55(-12)$ & $2.52(-12)$ & 0.21 & $1.73(+6)$ & $1.71(+6)$ & 4 & \\
\hline & & & & 0.72 & $\mathrm{C}$ & $3.66(-12)$ & $3.25(-12)$ & 0.26 & $2.49(+6)$ & $2.21(+6)$ & 4 & \\
\hline & & & & 0.02 & $\mathrm{C}$ & $2.91(-13)$ & $3.10(-13)$ & 0.17 & $1.98(+6)$ & $2.11(+6)$ & 6 & \\
\hline \multirow{2}{*}{ RV Lib ........ } & 97 & $3.3 /[5.3]$ & 276 & 0.96 & $\mathrm{H}$ & $1.75(-13)$ & $1.59(-13)$ & 0.72 & $2.41(+6)$ & $2.19(+6)$ & 3 & 4 \\
\hline & & & & & $\mathrm{C}$ & $3.20(-14)$ & $4.20(-14)$ & & $1.70(+5)$ & $2.20(+5)$ & 3 & 4 \\
\hline \multirow{2}{*}{ SS Boo ......... } & 98 & $1.31 / 3.28$ & 220 & 0.43 & $\mathrm{C}$ & $1.39(-13)$ & $1.20(-13)$ & 1.09 & $1.23(+6)$ & $1.06(+6)$ & 3 & 4 \\
\hline & & & & 0.33 & $\mathrm{C}$ & $1.44(-13)$ & $1.22(-13)$ & 1.41 & $1.27(+6)$ & $1.08(+6)$ & 5 & 4 \\
\hline \multirow[t]{4}{*}{ RT CrB ........ } & 103 & $2.65 / 2.65$ & 360 & 0.73 & $\mathrm{H}$ & $1.52(-14)$ & $2.03(-14)$ & 0.18 & $5.44(+5)$ & $7.25(+5)$ & 3 & 4 \\
\hline & & & & & $\mathrm{C}$ & $3.39(-14)$ & $4.65(-14)$ & 0.33 & $1.23(+6)$ & $1.67(+6)$ & 3 & 4 \\
\hline & & & & 0.80 & $\mathrm{H}$ & $3.09(-14)$ & $1.16(-14)$ & 0.64 & $1.13(+6)$ & $4.35(+5)$ & 5 & 4 \\
\hline & & & & & $\mathrm{C}$ & $6.92(-14)$ & $4.36(-14)$ & 1.07 & $2.50(+6)$ & $1.60(+6)$ & 5 & 4 \\
\hline \multirow[t]{6}{*}{ Ww Dra ....... } & 109 & $2.3 / 3.9$ & 180 & 0.87 & $\mathrm{H}$ & $6.35(-14)$ & $3.65(-14)$ & 0.19 & $7.31(+5)$ & $4.20(+5)$ & 3 & 4 \\
\hline & & & & & $\mathrm{C}$ & $3.29(-13)$ & $2.29(-13)$ & 0.68 & $3.79(+6)$ & $2.64(+6)$ & 3 & 4 \\
\hline & & & & 0.63 & $\mathbf{H}$ & $4.63(-14)$ & & 0.29 & $5.57(+5)$ & & 5 & 4 \\
\hline & & & & & $\mathrm{C}$ & $2.95(-13)$ & $2.29(-13)$ & 0.68 & $1.24(+6)$ & $9.57(+5)$ & 5 & 4 \\
\hline & & & & 0.31 & $\mathrm{H}$ & $6.84(-14)$ & $5.05(-14)$ & 0.23 & $8.23(+5)$ & $6.08(+5)$ & 6 & \\
\hline & & & & & $\mathrm{C}$ & $3.45(-13)$ & $3.66(-13)$ & 0.54 & $1.44(+6)$ & $1.53(+6)$ & 6 & \\
\hline HR 6469 & 113 & l & 50 & 0.81 & $\ldots$ & $7.03(-13)$ & $7.81(-13)$ & 0.22 & $* 2.48(+5)$ & $2.75(+5)$ & 6 & \\
\hline Z Her & 117 & $1.69 / 2.60$ & 75 & & $\mathrm{C}$ & $8.76(-13)$ & $7.02(-13)$ & 0.58 & $1.43(+6)$ & $1.14(+6)$ & 6 & 5 \\
\hline \multirow[t]{3}{*}{ MM Her ........... } & 118 & $1.58 / 2.83$ & 190 & 0.27 & $\mathrm{H}$ & $5.09(-14)$ & $2.85(-14)$ & 0.45 & $1.45(+6)$ & $8.10(+5)$ & 5 & 1 \\
\hline & & & & & $\mathrm{C}$ & $3.01(-13)$ & $2.24(-13)$ & 1.53 & $2.67(+6)$ & $1.98(+6)$ & 5 & 1 \\
\hline & & & & 0.98 & $\mathrm{~T}$ & $3.45(-13)$ & $3.34(-13)$ & 1.82 & & & 6 & 1 \\
\hline AW Her & 125 & $1.12 / 3.0$ & 240 & 0.91 & $\mathrm{C}$ & $8.33(-14)$ & $7.75(-14)$ & 3.65 & $1.05(+6)$ & $9.75(+5)$ & 5 & \\
\hline 42 Cap. & 151 & I & 34 & 0.18 & $\ldots$ & $3.66(-12)$ & $2.93(-12)$ & 0.76 & ${ }^{*} 1.29(+6)$ & $1.03(+6)$ & 5 & \\
\hline \multirow[t]{6}{*}{ RT Lac } & 153 & $3.4 / 4.2$ & 205 & 0.87 & $\mathrm{H}$ & $3.02(-13)$ & $2.27(-13)$ & & $2.16(+6)$ & $1.62(+6)$ & 2 & 5 \\
\hline & & & & & $\mathrm{C}$ & $2.07(-13)$ & $1.94(-13)$ & & $9.69(+5)$ & $9.08(+5)$ & 2 & 5 \\
\hline & & & & 0.97 & $\mathrm{~T}$ & $5.31(-13)$ & $4.98(-13)$ & 3.31 & & & 5 & 5 \\
\hline & & & & 0.55 & $\mathrm{~T}$ & $4.29(-13)$ & $3.93(-13)$ & 1.81 & & & 5 & 5 \\
\hline & & & & 0.12 & $\hat{\mathrm{H}}$ & $4.03(-13)$ & $2.82(-13)$ & 0.96 & $2.88(+6)$ & $2.02(+6)$ & 6 & 5 \\
\hline & & & & & $\mathrm{C}$ & $4.12(-13)$ & $2.75(-13)$ & 0.88 & $1.93(+6)$ & $1.29(+6)$ & 6 & 5 \\
\hline AR Lac. & 155 & $1.8 / 3.1$ & 47 & 0.95 & $\mathrm{C}$ & $4.19(-12)$ & $3.53(-12)$ & 0.78 & $1.89(+6)$ & $1.59(+6)$ & 6 & \\
\hline & & & & 0.95 & C & $5.13(-12)$ & $4.68(-12)$ & 0.96 & $2.32(+6)$ & $2.12(+6)$ & 6 & \\
\hline SZ Psc $\ldots \ldots \ldots$ & 164 & $1.38 / 5.08$ & 100 & 0.42 & $\mathrm{C}$ & $3.69(-12)$ & $3.36(-12)$ & 0.72 & $2.81(+6)$ & $2.56(+6)$ & 2 & \\
\hline & & & & 0.57 & C & $5.39(-12)$ & $3.24(-12)$ & 1.14 & $4.10(+6)$ & $2.47(+6)$ & 5 & \\
\hline & & & & 0.32 & $\mathrm{C}$ & $3.59(-12)$ & $2.91(-12)$ & 0.84 & $2.73(+6)$ & $2.22(+6)$ & 6 & \\
\hline
\end{tabular}

REFERENCES._-(1) Montes et al. 1993; (2) Fernández-Figueroa et al. 1986b; (3) Fernández-Figueroa et al. 1986a; (4) De Castro et al. 1990; (5) Armentia et al. 1990 . 
TABLE 5

Ca II H AND K EMISSION-Line FluXes (Group 3)

\begin{tabular}{|c|c|c|c|c|c|c|c|c|c|c|c|}
\hline Name & $S$ & $R\left(R_{\odot}\right)$ & $d(\mathrm{pc})$ & $\varphi$ & Emission & $F_{\text {obs }}(\mathrm{K})$ & $F_{\text {obs }}(\mathrm{H})$ & $C(\mathrm{~K})$ & $F_{\mathrm{S}}(\mathrm{K})$ & $F_{\mathrm{S}}(\mathrm{H})$ & $\mathrm{O}$ \\
\hline 33 Psc & 1 & / & 27 & 0.19 & $\ldots$ & $4.58(-13)$ & $1.60(-13)$ & 0.09 & & & 2 \\
\hline$\zeta$ And . & 7 & $0.7 / 12.6$ & 31 & 0.29 & $\ldots$ & $1.38(-11)$ & $1.12(-11)$ & 0.98 & $1.64(+5)$ & $1.32(+5)$ & 7 \\
\hline \multirow[t]{4}{*}{$12 \mathrm{Cam} . . . \ldots \ldots$} & 40 & 1 & 134 & 0.51 & $\ldots$ & $1.77(-11)$ & $1.74(-11)$ & 1.43 & ${ }^{*} 2.51(+6)$ & $2.48(+6)$ & 2 \\
\hline & & & & 0.52 & $\ldots$ & $1.84(-11)$ & $1.73(-11)$ & 1.38 & $*^{*} .61(+6)$ & $2.47(+6)$ & 2 \\
\hline & & & & 0.57 & $\ldots$ & $1.62(-11)$ & $1.59(-11)$ & 1.36 & $* 2.30(+6)$ & $2.26(+6)$ & 2 \\
\hline & & & & 0.57 & $\ldots$ & $1.63(-11)$ & $1.57(-11)$ & 1.36 & ${ }^{*} 2.31(+6)$ & $2.24(+6)$ & 2 \\
\hline CQ Aur. & 48 & $1.9 / 8.7$ & 220 & 0.42 & $\mathrm{C}$ & $4.19(-13)$ & $4.58(-13)$ & 0.32 & $5.26(+5)$ & $5.76(+5)$ & 2 \\
\hline \multirow[t]{4}{*}{$\sigma$ Gem $\ldots \ldots \ldots \ldots$} & 60 & 1 & 59 & 0.82 & $\ldots$ & $3.03(-11)$ & $2.43(-11)$ & 1.73 & $* 5.81(+5)$ & $4.66(+5)$ & 4 \\
\hline & & & & 0.82 & $\ldots$ & $3.07(-11)$ & $2.56(-11)$ & 1.82 & $* 5.89(+5)$ & $4.91(+5)$ & 4 \\
\hline & & & & 0.92 & $\ldots$ & $2.98(-11)$ & $2.50(-11)$ & 1.81 & $* 5.71(+5)$ & $4.80(+5)$ & 4 \\
\hline & & & & 0.41 & $\ldots$ & $4.20(-11)$ & $3.20(-11)$ & 1.81 & $* 8.06(+5)$ & $6.14(+5)$ & 7 \\
\hline RZ Cnc & 66 & $10.2 / 12.2$ & 395 & 0.36 & $\mathbf{H}$ & $4.63(-13)$ & $4.57(-13)$ & 1.26 & $9.53(+5)$ & $9.42(+5)$ & 4 \\
\hline \multirow[t]{2}{*}{93 Leo ......... } & 81 & $1.7 / 5.9$ & 36 & $\ldots$ & $\mathrm{C}$ & $6.93(-12)$ & $7.62(-12)$ & 0.28 & $3.88(+5)$ & $4.28(+5)$ & 4 \\
\hline & & & & & $\mathrm{C}$ & $6.43(-12)$ & $8.09(-12)$ & 0.28 & $4.71(+5)$ & $5.92(+5)$ & 4 \\
\hline \multirow[t]{4}{*}{ DK Dra ......... } & 83 & $\geq 13 / \geq 13$ & 130 & 0.44 & $\ldots$ & $6.82(-12)$ & $6.21(-12)$ & 1.78 & $1.34(+6)$ & $1.22(+6)$ & 2 \\
\hline & & & & 0.10 & $\ldots$ & $7.92(-12)$ & $7.29(-12)$ & 2.70 & $1.56(+6)$ & $1.43(+6)$ & 4 \\
\hline & & & & 0.13 & $\ldots$ & $8.09(-12)$ & $6.22(-12)$ & 2.63 & $1.59(+6)$ & $1.22(+6)$ & 4 \\
\hline & & & & 0.13 & $\ldots$ & $6.87(-12)$ & $6.94(-12)$ & 2.07 & $1.35(+6)$ & $1.36(+6)$ & 4 \\
\hline \multirow[t]{2}{*}{ GX Lib ......... } & 100 & $1 \geq 7$ & [219] & 0.36 & $\mathrm{C}$ & $5.09(-13)$ & $4.19(-13)$ & 1.63 & $* 9.79(+5)$ & $8.06(+5)$ & 6 \\
\hline & & & & 0.44 & $\mathrm{C}$ & $3.30(-13)$ & $2.73(-13)$ & 1.35 & *6.35 (+5) & $5.25(+5)$ & 6 \\
\hline$\epsilon \mathrm{UMi}$ & 110 & $1.7 / 12$ & 71 & 0.43 & $\mathrm{C}$ & $4.04(-12)$ & $2.91(-12)$ & 0.40 & $2.78(+5)$ & $2.00(+5)$ & 6 \\
\hline V792 Her & 111 & $2.58 / 12.28$ & 310 & 0.10 & $\mathrm{C}$ & $9.26(-13)$ & $7.70(-13)$ & 1.54 & $1.16(+6)$ & $9.65(+5)$ & 6 \\
\hline DR Dra ........ & 115 & $0.012 / \geq 5$ & 88 & $\ldots$ & $\mathrm{C}$ & $3.09(-12)$ & $2.77(-12)$ & 2.41 & $* 1.88(+6)$ & $1.69(+6)$ & 6 \\
\hline o Dra ... & 127 & 1 & 67 & 0.66 & $\ldots$ & $2.24(-12)$ & $1.63(-12)$ & 0.69 & $* 2.50(+5)$ & $1.82(+5)$ & 6 \\
\hline V1762 Cyg ...... & 132 & $\geq 8$ & 48 & 0.45 & $\ldots$ & $4.35(-12)$ & $3.78(-12)$ & 2.00 & $* 1.46(+6)$ & $1.27(+6)$ & 6 \\
\hline HR 7428 . & 136 & 1 & 302 & 0.52 & $\mathrm{C}$ & $1.11(-12)$ & $1.15(-12)$ & 0.34 & $* 4.07(+5)$ & $4.21(+5)$ & 6 \\
\hline V1764 Cyg. & 137 & $\geq 22$ & 340 & & & $7.18(-13)$ & $7.36(-12)$ & 1.00 & *3.37(+5) & $3.46(+5)$ & 6 \\
\hline HK Lac ......... & 154 & 1 & 150 & 0.89 & $\mathrm{C}$ & $3.18(-12)$ & $2.81(-12)$ & 2.42 & *2.99(+6) & $2.64(+6)$ & 6 \\
\hline V350 Lac ........ & 157 & $\geq 11$ & 69 & 0.50 & $\ldots$ & $2.23(-12)$ & $1.92(-12)$ & 1.74 & $* 1.03(+6)$ & $8.91(+5)$ & 6 \\
\hline \multirow[t]{2}{*}{ IM Peg ............ } & 159 & $1 \geq 12$ & 50 & 0.45 & $\mathrm{C}$ & $1.47(-11)$ & $1.16(-11)$ & 2.47 & $* 1.17(+6)$ & $9.18(+5)$ & 5 \\
\hline & & & & 0.65 & $\mathrm{C}$ & $1.08(-11)$ & $9.76(-11)$ & 1.89 & $* 8.55(+5)$ & $7.71(+5)$ & 6 \\
\hline \multirow[t]{2}{*}{$\lambda$ And $\ldots \ldots \ldots$} & 166 & 1 & 23 & 0.56 & $\ldots$ & $4.47(-11)$ & $4.15(-11)$ & 2.30 & $* 1.15(+6)$ & $1.07(+6)$ & 6 \\
\hline & & & & 0.10 & $\ldots$ & $5.07(-11)$ & $4.62(-11)$ & 2.16 & $* 1.30(+6)$ & $1.19(+6)$ & 7 \\
\hline
\end{tabular}

was 1.3 times more active than the primary component. At present the activity level of both components is lower, and of nearly the same intensity, in agreement with the results of Bopp (1984). All these results suggest that the activity level of this system varies with time.

In addition, one observation of $\mathrm{H} \alpha$ was carried out at 0.05 phase where the line was an absorption feature.

\subsubsection{V772 Herculis (HD 165590)}

This is another triple system whose components are latetype main-sequence stars. The primary of the visual pair (ADS 11060 ) is a single-lined spectroscopic binary ( $\mathrm{Aa}, \mathrm{Ab})$. The $\mathrm{Ca}$ II spectra showed well defined emissions from the two more luminous stars (Aa, B). Significant variations with time of the emission fluxes were detected in the B component (G5 V).

One spectrum in the $\mathrm{H} \alpha$ region exhibits strong absorption. Because of the orbital phase $(0.46)$ it was not possible to separate the contribution of each component. The calculated ratio between the continuum flux of both components in this spectral region is $f_{H} / f_{C}=1.92$.

\subsubsection{V815 Herculis (HD 166181)}

This single-lined spectroscopic binary was classified by $\mathrm{Fe}$ kel et al. (1986) as an early-type BY Dra. Six new images of Ca
II $\mathrm{H}$ and $\mathrm{K}$ at different phases are available for this system. The emission lines are unshifted with respect to the absorption, so we can again confirm that the hot star is the active component. The measured emission fluxes do not show variations with orbital phase, so that only one value is given in Table 3. Furthermore, surface fluxes presented in this work are comparable to those given by Bopp (1984) and Fernández-Figueroa et al. (1986a) therefore we can conclude that chromospheric activity of V815 Her was practically constant during this interval of time.

One observation of the $\mathrm{H} \alpha$ line at 0.52 phase (Fig. 7 below) presents a filled-in absorption profile in according with the behavior pointed out by Xuefu \& Huisong (1986).

\subsubsection{BY Draconis (HD 234677)}

Our four observations of this system clearly show that both components are active with the hot component having the stronger $\mathrm{Ca}$ II emission. The two components also show $\mathrm{H} \epsilon$ in emission. In order to measure the emission fluxes a Gaussian fit allowed us to separate the four emission lines (Fig. 3). The emission flux values are slightly higher than those given by Bopp ( 1984) because his spectrogram has an unresolved Ca II emission which is coming from both components. A possible orbital phase variation of the $\mathrm{Ca}$ II emission line fluxes is suggested. 
TABLE 6

H $\epsilon$ EMISSION-Line FluXes (Groups 1, 2, AND 3)

\begin{tabular}{|c|c|c|c|c|c|c|c|c|c|}
\hline Name & $S$ & $R\left(R_{\odot}\right)$ & $d(\mathrm{pc})$ & $\varphi$ & Emission & $F_{\text {obs }}(\mathrm{H} \epsilon)$ & $C(\mathrm{H} \epsilon)$ & $F_{\mathrm{S}}(\mathrm{H} \epsilon)$ & $\mathrm{O}$ \\
\hline \multicolumn{10}{|c|}{ Group 1} \\
\hline \multirow[t]{2}{*}{ DH Leo $\ldots . . .}$. & 73 & $0.97 / 0.67$ & 32 & 0.32 & $\mathrm{H}$ & $1.64(-13)$ & 0.15 & $3.51(+5)$ & 4 \\
\hline & & & & 0.55 & $\mathrm{H}$ & $1.37(-13)$ & 0.12 & $2.43(+5)$ & 4 \\
\hline \multirow{8}{*}{ BY Dra ........ } & 126 & $1.2 /$ & 16 & 0.71 & $\mathrm{H}$ & $3.72(-13)$ & 0.62 & $1.23(+5)$ & 5 \\
\hline & & & & & $\mathrm{C}$ & $1.04(-13)$ & & & 5 \\
\hline & & & & 0.73 & $\mathbf{H}$ & $3.19(-13)$ & 0.62 & $1.06(+5)$ & 5 \\
\hline & & & & & $\mathrm{C}$ & $8.43(-12)$ & & & 5 \\
\hline & & & & 0.22 & $\mathrm{H}$ & $3.65(-13)$ & 0.69 & $1.21(+5)$ & 5 \\
\hline & & & & & $\mathrm{C}$ & $2.97(-13)$ & & & 5 \\
\hline & & & & 0.23 & $\mathrm{H}$ & $3.56(-13)$ & 0.57 & $1.18(+5)$ & 5 \\
\hline & & & & & $\mathrm{C}$ & $1.91(-13)$ & & & 5 \\
\hline \multirow[t]{5}{*}{ V775 Her ....... } & 129 & $0.85 /$ & 24 & 0.04 & $\mathrm{H}$ & $6.64(-13)$ & 0.76 & $1.04(+6)$ & 5 \\
\hline & & & & 0.06 & $\mathbf{H}$ & $5.78(-13)$ & 0.66 & $9.06(+6)$ & 5 \\
\hline & & & & 0.37 & $\mathrm{H}$ & $4.10(-13)$ & 0.44 & $6.42(+5)$ & 5 \\
\hline & & & & 0.40 & $\mathbf{H}$ & $5.35(-13)$ & 0.72 & $8.38(+5)$ & 5 \\
\hline & & & & 0.07 & $\mathrm{H}$ & $2.66(-13)$ & 0.29 & $4.17(+5)$ & 5 \\
\hline \multirow[t]{3}{*}{ V478 Lyr ...... } & 131 & $\geq 0.9 /$ & 26 & 0.40 & $\mathrm{H}$ & & & & 5 \\
\hline & & & & 0.85 & $\mathrm{H}$ & & & & 5 \\
\hline & & & & 0.77 & $\mathrm{H}$ & & & & 5 \\
\hline \multirow[t]{2}{*}{$\mathrm{KZ}$ And } & 162 & $\geq 0.74$ & 23 & 0.33 & $\mathrm{P}$ & & & & 6 \\
\hline & & & & & $S$ & $1.66(-13)$ & 0.23 & $3.15(+5)$ & 6 \\
\hline \multicolumn{10}{|c|}{ Group 2} \\
\hline \multirow[t]{3}{*}{ AR Psc $\ldots \ldots \ldots$} & 15 & $\geq 1.5$ & 70 & 0.33 & $\mathrm{C}$ & $1.38(-13)$ & 0.21 & $6.01(+5)$ & 2 \\
\hline & & & & 0.39 & $\mathrm{C}$ & $1.42(-13)$ & 0.28 & $6.19(+5)$ & 2 \\
\hline & & & & 0.67 & $\mathrm{C}$ & $1.24(-13)$ & 0.17 & $5.42(+5)$ & 2 \\
\hline \multirow[t]{5}{*}{ V711 Tau ...... } & 27 & $1.3 / 3.9$ & 36 & 0.16 & $\mathbf{H}$ & & & & 2 \\
\hline & & & & & $\mathrm{C}$ & $1.25(-11)$ & 0.35 & $2.10(+6)$ & 2 \\
\hline & & & & 0.57 & $\ldots$ & $1.00(-11)$ & 0.32 & $1.68(+6)$ & 2 \\
\hline & & & & 0.46 & $\ldots$ & $4.73(-12)$ & 0.19 & $7.91(+5)$ & 4 \\
\hline & & & & 0.83 & $\ldots$ & $3.28(-12)$ & 0.16 & $5.48(+5)$ & 4 \\
\hline AW Her $\ldots . . .$. & 125 & $1.12 / 3.0$ & 240 & 0.91 & $\mathrm{C}$ & & & & 5 \\
\hline
\end{tabular}

Group 3

\begin{tabular}{lllllll}
\hline HK Lac ........ & 154 & $/$ & 150 & 0.89 & C & 6 \\
\hline
\end{tabular}

\subsubsection{V775 Herculis (HD 175742)}

Bopp et al. (1983) classifies this star as a BY Dra variable rather than an $\mathrm{RS} \mathrm{CVn} \mathrm{binary} \mathrm{due} \mathrm{to} \mathrm{its} \mathrm{K} 0 \mathrm{~V}$ spectral type.

In our observations this system presents strong $\mathrm{Ca}$ II $\mathrm{H}$ and $\mathrm{K}$ emissions always centered at the absorption line, therefore this emission corresponds to the hot component $(\mathrm{K} 0 \mathrm{~V})$. The $\mathrm{H} \epsilon$ line appears in emission. The measured surface fluxes in our five observations seem to be independent of the orbital phase and they are slightly lower than those reported by Strassmeier et al. (1990). This discrepancy may be caused by the different measuring methods of the emission fluxes.

\subsubsection{V478 Lyrae (HD 178450)}

This single-lined spectroscopic binary has been classified as an early-type BY Dra (Fekel 1988). Our three observations at phases $0.40,0.85$, and 0.77 show strong $\mathrm{H}$ and $\mathrm{K}$ emissions and indicate that the emission fluxes change slightly with orbital phase. $\mathrm{H} \epsilon$ emission seems to be present in our spectrograms but noise makes it difficult to measure the flux and for this reason the values are not given in Table 3 .

\subsubsection{KZ Andromedae (HD 218738)}

This double-lined ( $\mathrm{dK} 2 / \mathrm{dK} 2)$ system has been observed at 0.33 phase and presents two Ca II emissions, one from each component. In the case of $\mathrm{K}$ line the intensities of both emissions are nearly equal as expected of identical components with synchronized rotation. In the $3968 \AA$ region, three emissions are clearly seen, that is, in order of increasing wavelength: $\mathrm{Ca}$ II $\mathrm{H}$ of the primary, $\mathrm{Ca}$ II $\mathrm{H}$ of the secondary overlapped with $\mathrm{H} \epsilon$ emission of the primary and finally the $\mathrm{H} \epsilon$ of the secondary (Fig. 4). Therefore the $\mathrm{H}$ line measured emission flux of the secondary includes the contribution of $\mathrm{H} \epsilon$ primary component. Taking into account that the components are identical, the values in Tables 3 and 6 are consistent and they indicate that both components show an identical activity level.

\subsection{Group 2 (Active Component of Luminosity Class IV)}

\subsubsection{AR Piscium (HD 8357)}

This system is a double-lined spectroscopic binary. Three spectra in the region of the $\mathrm{Ca}$ II $\mathrm{H}$ and $\mathrm{K}$ lines have been taken, 
TABLE 7

H $\alpha$ Equivalent WidTHS (GrouPs 1, 2, AND 3)

\begin{tabular}{|c|c|c|c|c|c|c|c|c|}
\hline $\begin{array}{l}\text { Name } \\
\text { (1) }\end{array}$ & $\begin{array}{c}S \\
(2)\end{array}$ & $\begin{array}{l}\text { Emission } \\
\text { (3) }\end{array}$ & $\begin{array}{c}\varphi \\
(4)\end{array}$ & $\underset{(5)}{\operatorname{FWHM}(\AA)}$ & $\begin{array}{c}\mathrm{EW} \alpha(\AA) \\
(6)\end{array}$ & $\begin{array}{l}R_{c} \\
\text { (7) }\end{array}$ & $\begin{array}{c}\mathrm{EW}_{e}(\AA) \\
(8)\end{array}$ & $\begin{array}{l}\mathrm{O} \\
\text { (9) }\end{array}$ \\
\hline \multicolumn{9}{|c|}{ Group 1} \\
\hline $\begin{array}{l}13 \text { Get } \ldots \ldots \ldots \ldots \\
\sigma^{2} \mathrm{CrB} \ldots \ldots \ldots \ldots \\
\text { V772 } \ldots e r \ldots \ldots \\
\text { V815 Her } \ldots \ldots \ldots\end{array}$ & $\begin{array}{r}5 \\
107 \\
119 \\
122\end{array}$ & $\begin{array}{l}* \\
* \\
\mathrm{H}\end{array}$ & $\begin{array}{l}0.74 \\
0.05 \\
0.46 \\
0.52\end{array}$ & $\begin{array}{l}2.23 \\
4.01 \\
2.91 \\
2.62\end{array}$ & $\begin{array}{l}1.52 \\
1.19 \\
1.21 \\
0.79\end{array}$ & $\begin{array}{l}0.35 \\
0.68 \\
0.64 \\
0.74\end{array}$ & $\begin{array}{r}0.09 \\
-0.24 \\
0.02 \\
-0.44\end{array}$ & $\begin{array}{l}2 \\
6 \\
6 \\
6\end{array}$ \\
\hline
\end{tabular}

\begin{tabular}{|c|c|c|c|c|c|c|c|c|}
\hline \multicolumn{9}{|c|}{ Group 2} \\
\hline AR Psc $\ldots \ldots \ldots$ & 15 & $\mathrm{H}$ & 0.61 & 6.35 & 0.42 & 0.90 & & 2 \\
\hline & & $\mathrm{C}$ & & 1.41 & -0.69 & -0.73 & -1.81 & 2 \\
\hline V711 Tau & 27 & $\mathrm{C}$ & 0.57 & 3.75 & -1.47 & -0.64 & -2.69 & 2 \\
\hline $54 \mathrm{Cam}$......... & 61 & $\mathrm{H}$ & 0.08 & 2.08 & 0.60 & 0.73 & & 2 \\
\hline & & $\mathrm{C}$ & & 1.68 & 0.91 & 0.50 & & 2 \\
\hline RT CrB $\ldots \ldots \ldots$ & 103 & * & 0.55 & 3.17 & 1.52 & 0.54 & 0.15 & 6 \\
\hline WW Dra ........ & 109 & * & 0.53 & 2.84 & 0.99 & 0.57 & -0.32 & 6 \\
\hline HR $6469 \ldots \ldots$ & 113 & $*$ & & 1.94 & 1.14 & 0.42 & -0.40 & 6 \\
\hline Z Her ......... & 117 & $\mathrm{H}$ & 0.64 & 2.34 & 1.09 & 0.59 & & 6 \\
\hline & & $\mathrm{C}$ & & 2.51 & 0.80 & 0.72 & & 6 \\
\hline MM Her ........ & 118 & $\mathrm{H}$ & 0.24 & 2.31 & 1.03 & 0.78 & & 6 \\
\hline & & $\mathrm{C}$ & & 1.92 & 0.46 & 0.61 & & 6 \\
\hline RT Lac $\ldots \ldots \ldots$ & 153 & $\mathrm{C}$ & & & & & & 2 \\
\hline AR Lac ......... & 155 & $*$ & 0.40 & 3.52 & 1.49 & 0.63 & & 6 \\
\hline SZ Psc ......... & 164 & $\mathbf{H}$ & 0.84 & & 0.36 & 0.86 & & 6 \\
\hline & & $\mathrm{C}$ & & & 0.52 & 0.77 & & 6 \\
\hline \multicolumn{9}{|c|}{ Group 3} \\
\hline 33 Psc & 1 & $*$ & 0.18 & 1.35 & 1.12 & 0.28 & & 2 \\
\hline$\zeta$ And ........... & 7 & $*$ & 0.85 & 1.35 & 1.24 & 0.44 & & 4 \\
\hline $12 \mathrm{Cam} . . . \ldots \ldots$ & 40 & $*$ & 0.55 & 1.91 & 0.97 & 0.56 & -0.01 & 2 \\
\hline CQ Aur .......... & 48 & $*$ & 0.42 & 2.11 & 0.81 & 0.66 & -0.50 & 2 \\
\hline$\sigma$ Gem $\ldots \ldots \ldots$ & 60 & $*$ & 0.13 & 1.69 & 0.98 & 0.44 & & 4 \\
\hline GX Lib $\ldots \ldots \ldots$ & 100 & $*$ & 0.80 & 3.31 & 1.78 & 0.40 & & 6 \\
\hline$\epsilon \operatorname{Umi} \ldots \ldots \ldots \ldots$ & 110 & $\mathrm{C}$ & 0.53 & 2.14 & 1.35 & 0.37 & -0.18 & 6 \\
\hline \multirow[t]{2}{*}{ V792 Her ....... } & 111 & $\mathrm{H}$ & 0.21 & 1.08 & 0.23 & 0.81 & & 6 \\
\hline & & $\mathrm{C}$ & & 2.49 & 0.49 & 0.82 & & 6 \\
\hline DR Dra $\ldots \ldots \ldots$ & 115 & $\mathrm{C}$ & & 1.90 & 0.95 & 0.49 & -0.03 & 6 \\
\hline HK Lac ........ & 154 & $\mathrm{C}$ & & & & & & 6 \\
\hline V350 Lac ...... & 157 & $*$ & 0.62 & 2.07 & 1.13 & 0.48 & & 6 \\
\hline IM Peg $\ldots \ldots \ldots$ & 159 & $*$ & 0.85 & 1.63 & 0.57 & 0.67 & -0.38 & 6 \\
\hline$\lambda$ And $\ldots \ldots \ldots$ & 166 & $*$ & 0.81 & 1.77 & 1.00 & 0.43 & -0.11 & 6 \\
\hline
\end{tabular}

TABLE 8

H $\alpha$ REFERENCE STARS

\begin{tabular}{|c|c|c|c|c|}
\hline HD & Name & $T_{\mathrm{sp}}$ & $\begin{array}{c}\mathrm{EW} \alpha \\
(\AA)\end{array}$ & $R_{c}$ \\
\hline 194012 . & HR 7793 & F5 V & 1.54 & 0.25 \\
\hline 154417. & HR 6349 & F8 V & & 0.26 \\
\hline 136202 & $5 \mathrm{Ser}$ & F8 V & 1.43 & 0.22 \\
\hline 142373 & $\chi$ Her & F9 V & 1.45 & 0.30 \\
\hline 144287 & & G0 V & 1.15 & 0.23 \\
\hline 206860 . & HN Peg & G0 V & 1.36 & 0.31 \\
\hline 190406. & $15 \mathrm{Seg}$ & G1 V & 1.35 & 0.22 \\
\hline 143761 . & $\rho \mathrm{CrB}$ & G2 V & 1.31 & 0.21 \\
\hline 190360 . & HR 7670 & G6 IV & 1.22 & 0.22 \\
\hline $131156 \mathrm{~A}$ & $\chi$ Boo A & G8 V & 1.13 & 0.35 \\
\hline 190404 & & K0 V & 0.99 & 0.25 \\
\hline $201091 \ldots$ & 61 Cyg A & $\mathrm{K} 5 \mathrm{~V}$ & 0.79 & 0.35 \\
\hline 201091 . & 61 Cyg B & K7 V & 0.68 & 0.40 \\
\hline
\end{tabular}

in which strong $\mathrm{Ca} \Pi \mathrm{H}$ and $\mathrm{K}$ emissions can be seen. The emission features remain unshifted during the orbital period. This behavior points out that both, the emissions and continuum, belong to the cool component (G8 IV). As Bopp (1984) noted, the strong Balmer $\mathrm{H} \epsilon$ emission is remarkable.

In our spectrum the $\mathrm{H} \alpha$ line of the active component appears in emission above the continuum and overlapped with the absorption of the other component. We have performed a two-Gaussian fit (absorption and emission) in order to obtain the equivalent width (Fig. 7 below).

\subsubsection{V711 Tauri (HR 1099, HD 22468)}

V711 Tau is another double-lined spectroscopic binary, and the brightest of the intermediate-period RS CVn stars (Bopp \& Fekel 1976). Four spectra in the region of $\mathrm{Ca}$ II $\mathrm{H}$ and $\mathrm{K}$ have been taken at two different epochs. At 0.16 phase a Gaussian fit allowed us to separate the contribution from both compo- 


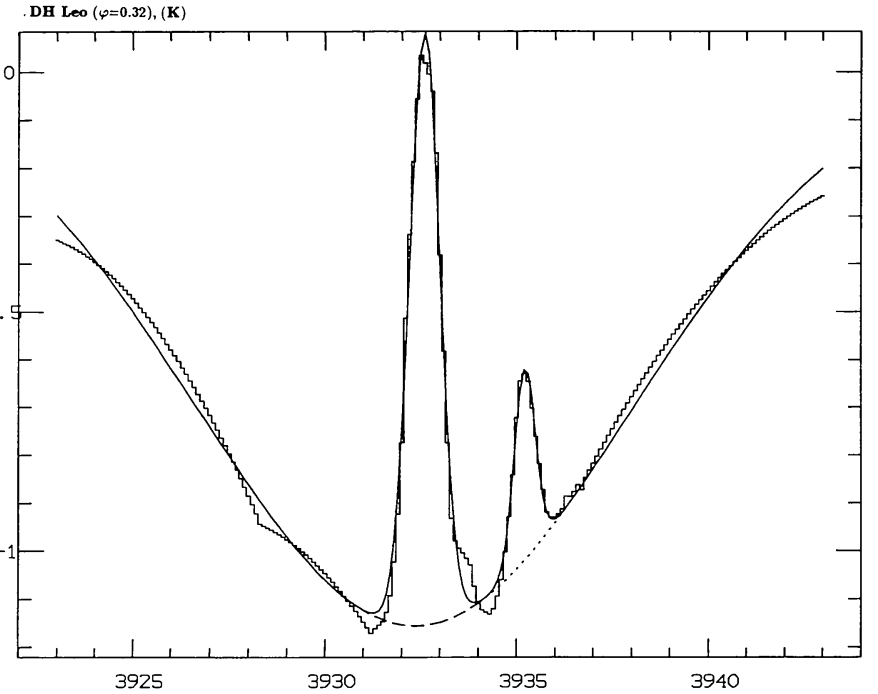

FIG. $1 a$

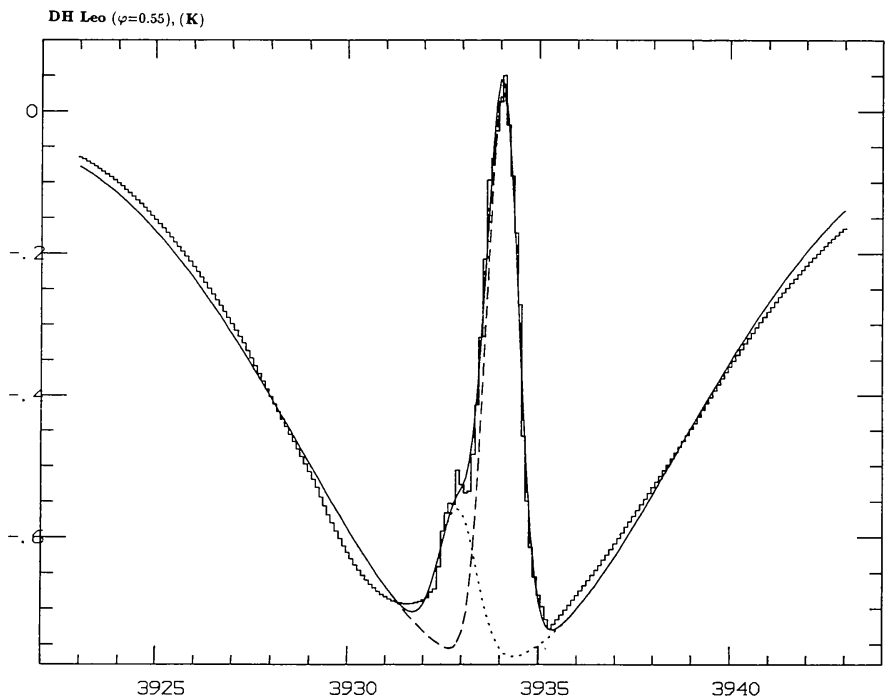

FIG. $1 c$

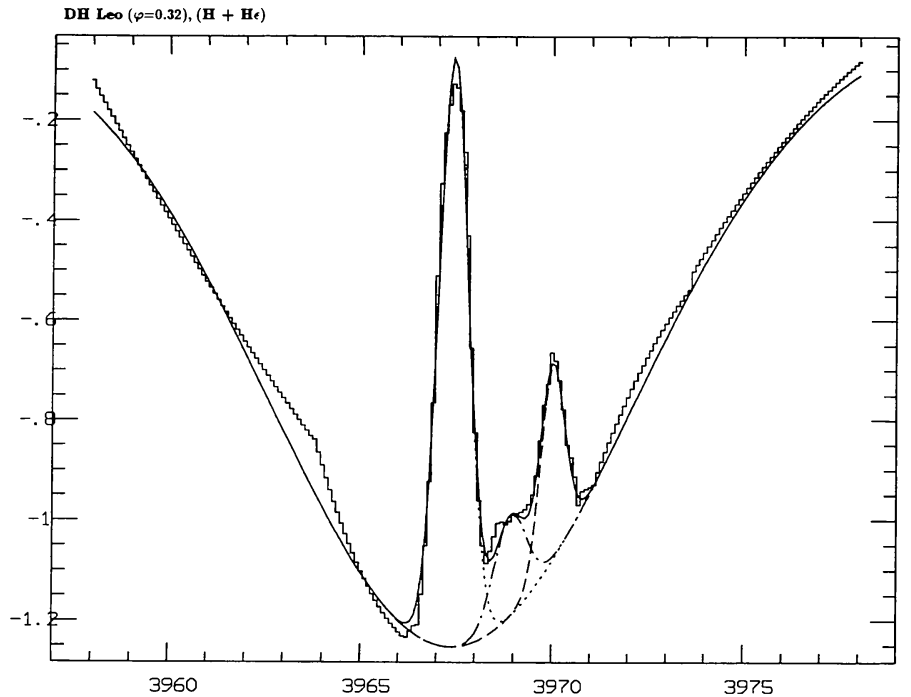

FIG. $1 b$

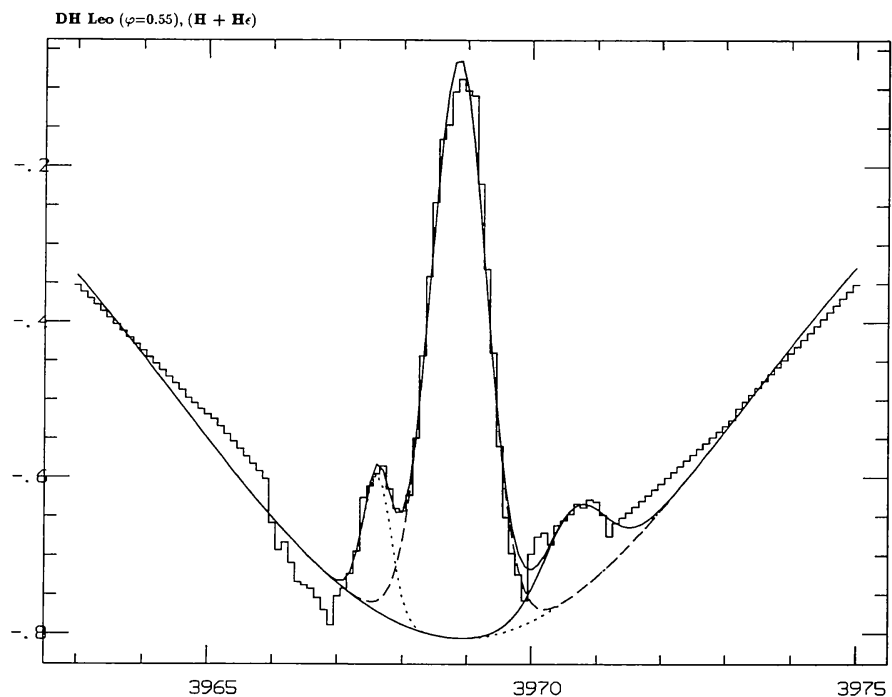

FIG. $1 d$

FIG. 1.-Gaussian fits to the Ca II $\mathrm{H}$ and $\mathrm{K}$ and the $\mathrm{H} \epsilon$ lines for DH Leo (at phases 0.32 , and 0.55 ). The dotted and dashed lines are the Gaussian fits and the continuous line is the observed spectrum.

nents (Fig. 5). The cool component is the more active star in the system. In the case of the $\mathrm{Ca}$ II $\mathrm{H}$ line the fit also provides a measurement of the $\mathrm{H} \epsilon$ emission, which comes only from the cool component. At the other orbital phases it was not possible to perform a similar deconvolution.

A trend of lower activity level can be inferred in the second epoch (1988), but the present observations are not enough to ascribe this variation to short-term (Buzasi, Huenemoerder, \& Ramsey 1991) or secular variability.

One spectrum of $\mathrm{H} \alpha$ line shows an emission feature visible above the continuum and the emission equivalent width computed is the largest in our sample.

\subsubsection{EI Eridani (HD 26337)}

One spectrum of the spotted RS CVn-type binary EI Eri at 0.94 phase in the region of the $\mathrm{Ca}$ II $\mathrm{H}$ and $\mathrm{K}$ lines, shows a moderate central emission as can be seen in Figure 6, in agreement with the moderate emission also reported by Fekel et al. (1986).

\subsubsection{Camelopardalis (AE Lynx, HD 65626)}

Our observations of $\mathrm{Ca} \mathrm{II} \mathrm{H}$ and $\mathrm{K}$ at four different phases show emissions from only one component. The emission lines are shifted compared to the $\mathrm{H}$ and $\mathrm{K}$ absorption features. 


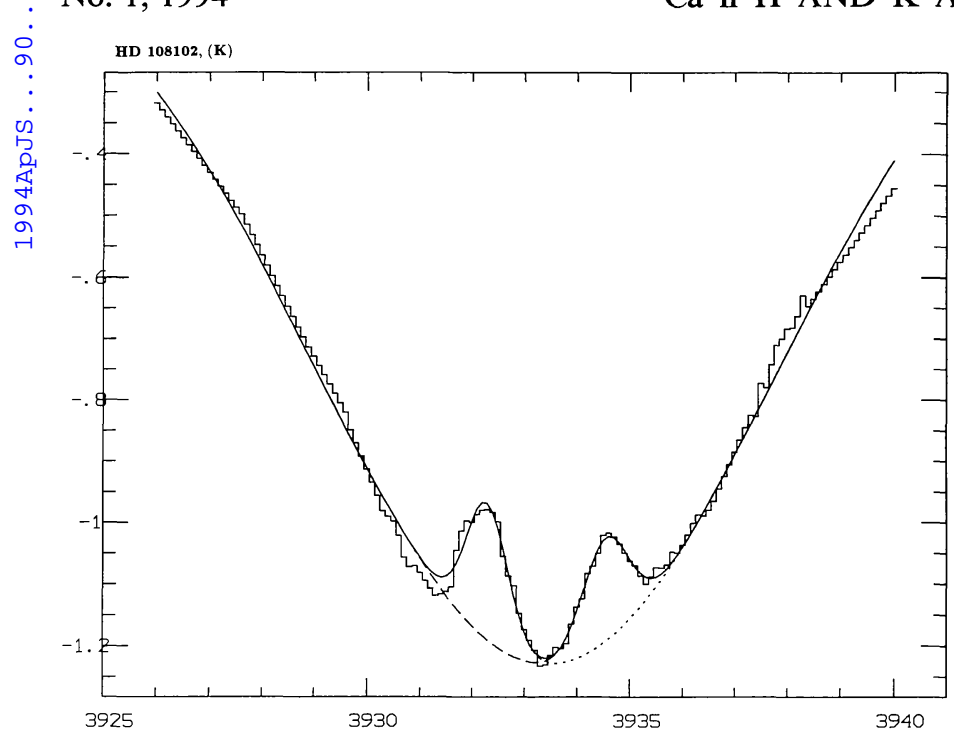

FIG. $2 a$

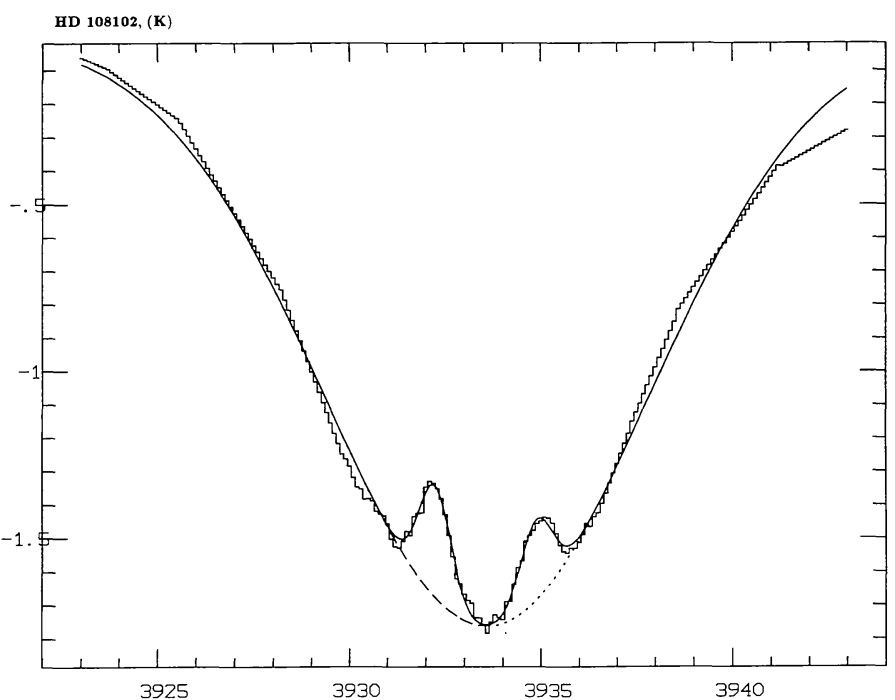

FIG. $2 c$

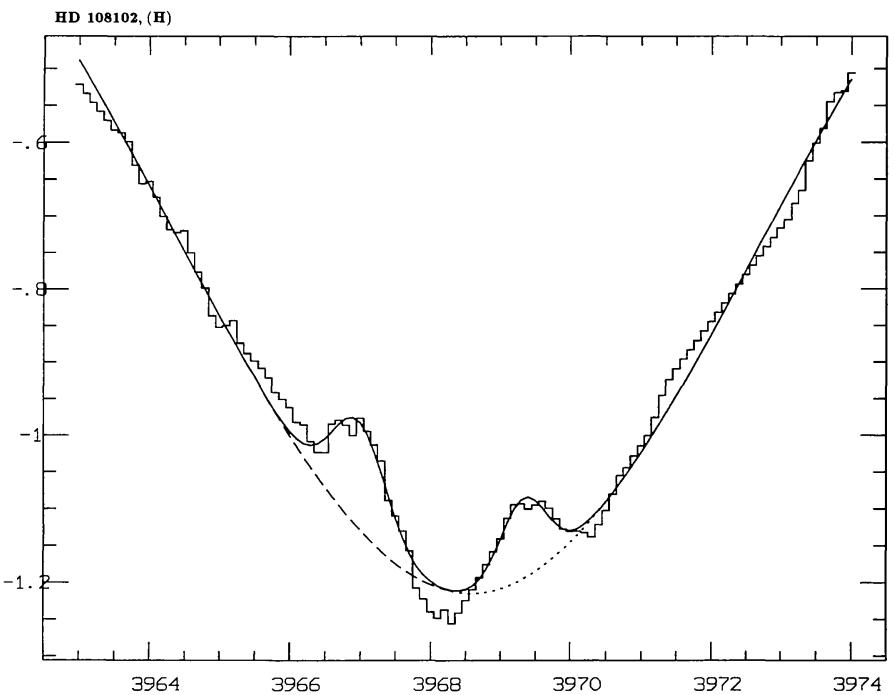

FIG. $2 b$

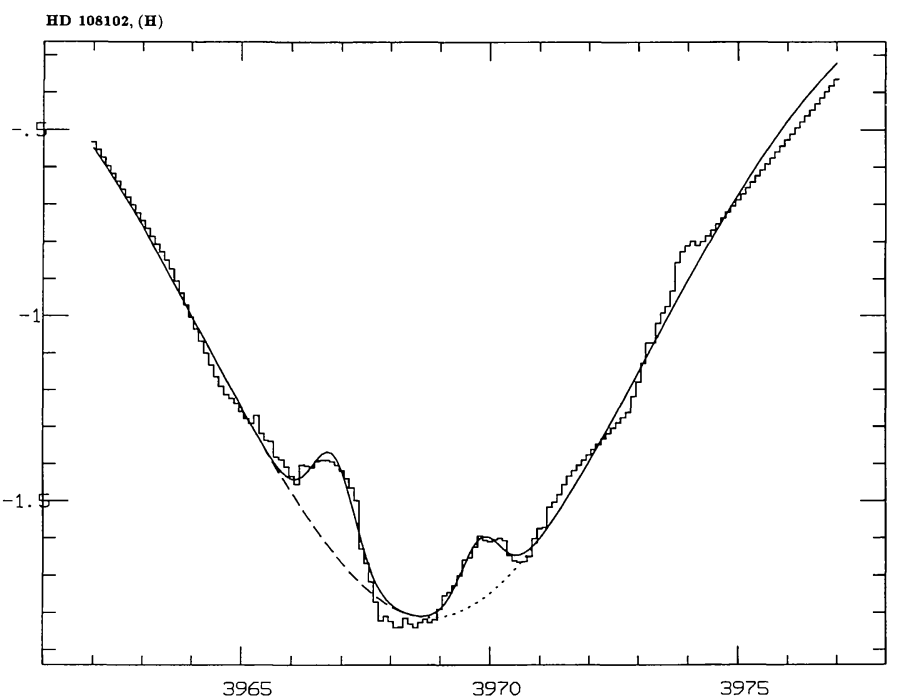

FIG. $2 d$

FIG. 2.-Gaussian fits to the $\mathrm{Ca}$ II $\mathrm{H}$ and $\mathrm{K}$ lines for $\mathrm{HD} 108102$. The dotted and dashed lines are the Gaussian fits and the continuous line is the observed spectrum.

Strassmeier \& Fekel (1990) noted that the hot star (F9 IV) is brighter than the cool star (G5 IV) in both the blue and the red wavelength regions. Therefore, the surface emission fluxes in Table 4 were computed using the data for the cool star. It is remarkable that the relative wavelength shifts between absorption and emission are in disagreement with the one computed using the orbital parameters of CABS.

One observation in the $\mathrm{H} \alpha$ region shows absorption lines from both components, again the computed orbital phase does not agree with the observed shift. The equivalent width of each star has been computed using the red magnitude difference between the hot and cool component given by Strassmeier \& Fekel (1990).

\subsubsection{RS Canum Venaticarum (HD 114519)}

This star, the prototype of the class being discussed, has been extensively studied by many authors. We have obtained two new observations in the region of $\mathrm{Ca}$ II $\mathrm{H}$ and $\mathrm{K}$ lines which confirm emission flux variations with the orbital phase found by us (Fernández-Figueroa et al. 1986a). The emission flux values are now higher which also indicates possible cyclical variations.

\subsubsection{RT Coronae Borealis (HD 139588)}

This is a double-lined spectroscopic system with moderate $\mathrm{Ca}$ II $\mathrm{H}$ and $\mathrm{K}$ emission from the two components, (De Castro 
BY Dra $(\varphi=0.71),(\mathbf{K})$

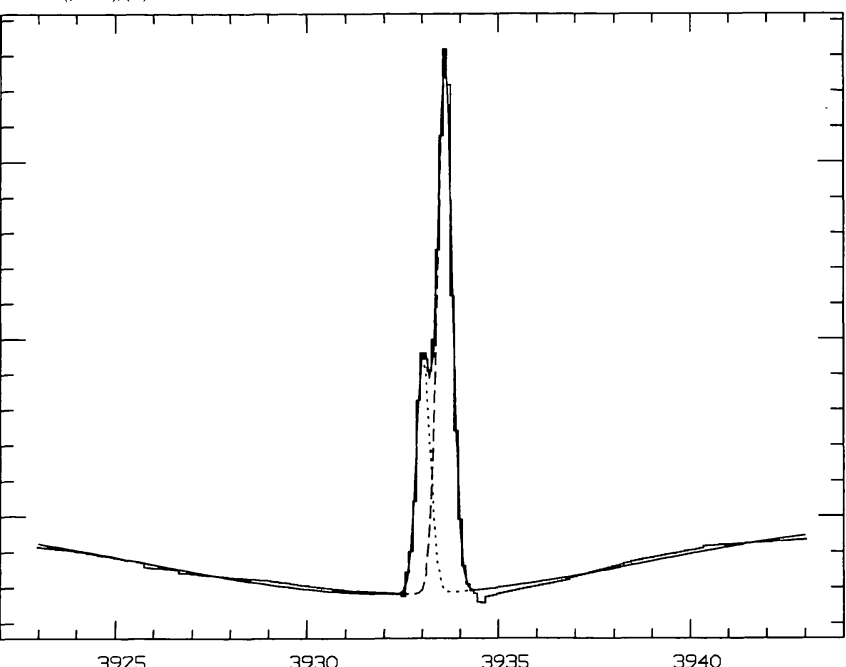

FIG. $3 a$

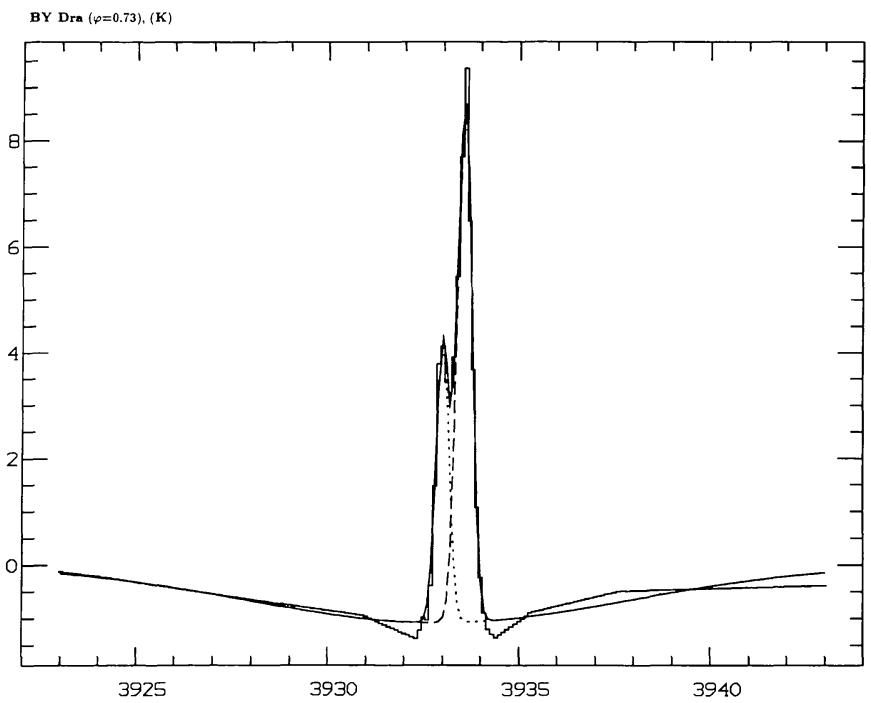

FIG. $3 c$

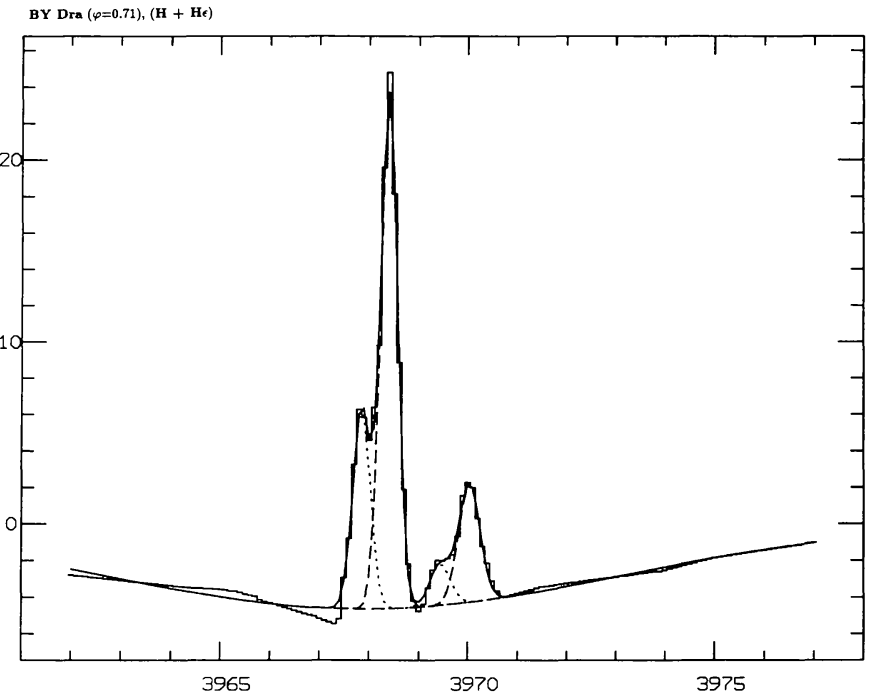

FIG. $3 b$

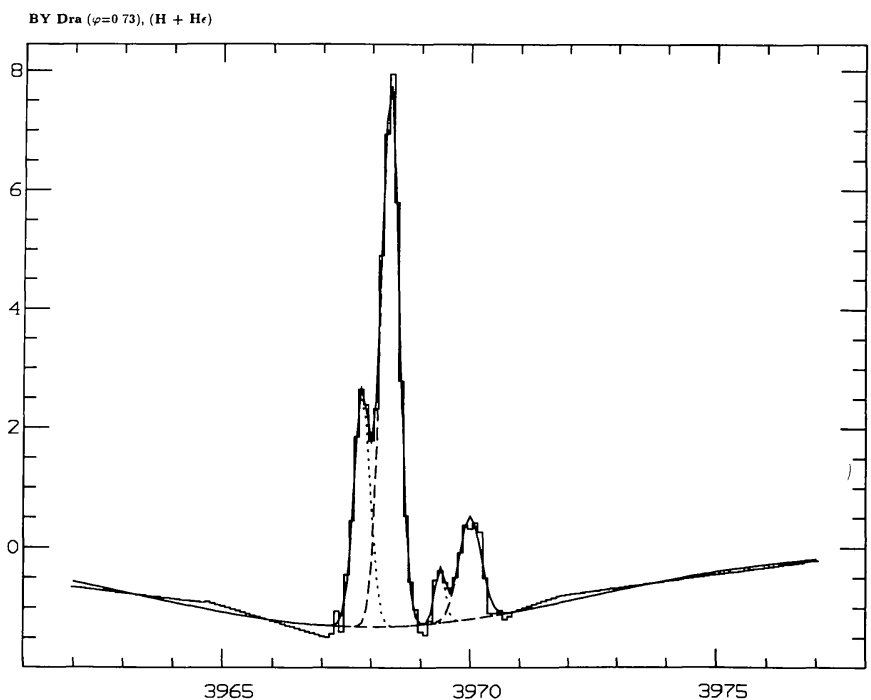

FIG. $3 d$

Fig. 3.-Gaussian fits to the $\mathrm{Ca}$ II $\mathrm{H}$ and $\mathrm{K}$ and the $\mathrm{H} \epsilon$ lines in BY Dra (at phases $0.71,0.73,0.22$, and 0.23 ). The dotted and dashed lines are the Gaussian fit and the continuous line is the observed spectrum.

et al. 1990). One observation of $\mathrm{H} \alpha$ line at 0.55 phase (Fig. 7) shows a strong absorption feature. The positive value obtained for the emission equivalent width indicates that this system has no filled-in absorption profile.

\subsubsection{WW Draconis (HD 150708)}

One observation of this double-lined system at phase 0.31 confirms that both components emit in $\mathrm{Ca}$ II $\mathrm{H}$ and $\mathrm{K}$. The strongest emission corresponds to the cool component as was pointed out by our previous observations (De Castro et al. 1990). One spectrum in the $\mathrm{H} \alpha$ region at 0.53 phase shows an absorption profile weakly filled-in by chromospheric emission.

\subsubsection{HR 6469 (V819 Herculis, HD 157482)}

HR 6469 is a spectroscopic triple system where the third star (G5 IV) is the active component. The closer pair formed by G0 V and F2 V stars is a eclipsing system. Three observations at different phases are available. A very faint emission seems to be always centered in the absorption, indicating that the emission is coming from the G5 IV component in accord with other observations (Bopp 1984; Strassmeier et al. 1990). A spectrum corresponding to phase 0.81 is shown in Figure 6 and their value of the $\mathrm{Ca}$ II $\mathrm{H}$ and $\mathrm{K}$ fluxes are listed in Table 4.

The $\mathrm{H} \alpha$ line shows a filled-in absorption profile in the observation carried out in 1989 July. 


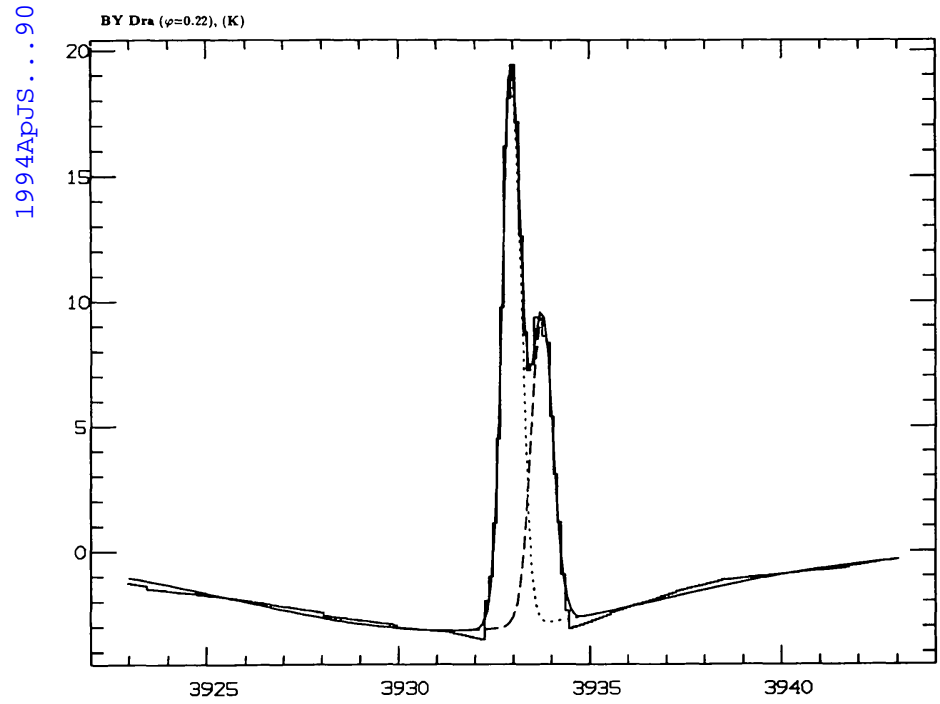

FIG. $3 e$

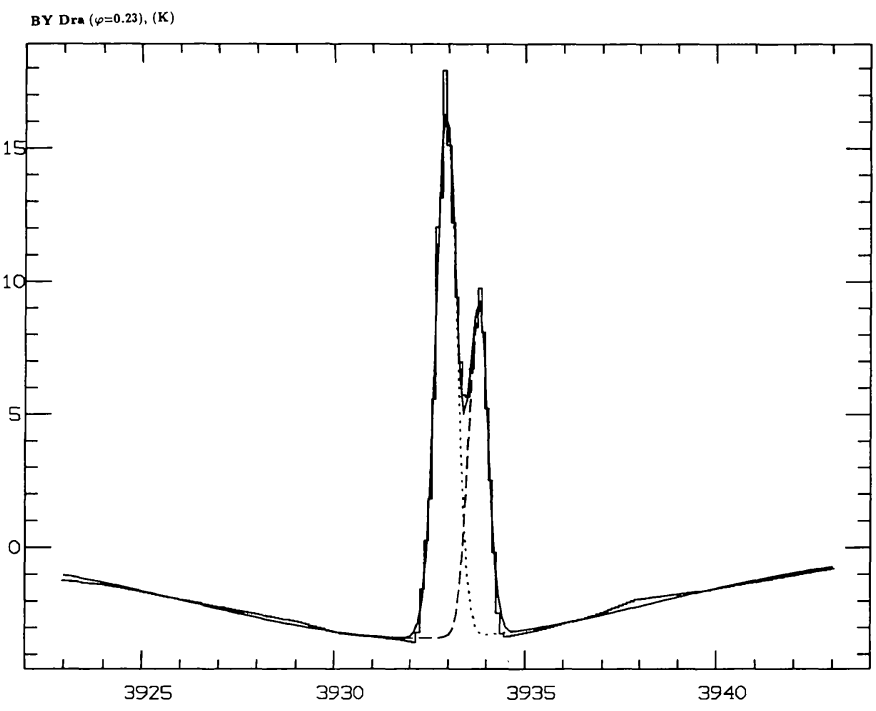

FIG. $3 g$

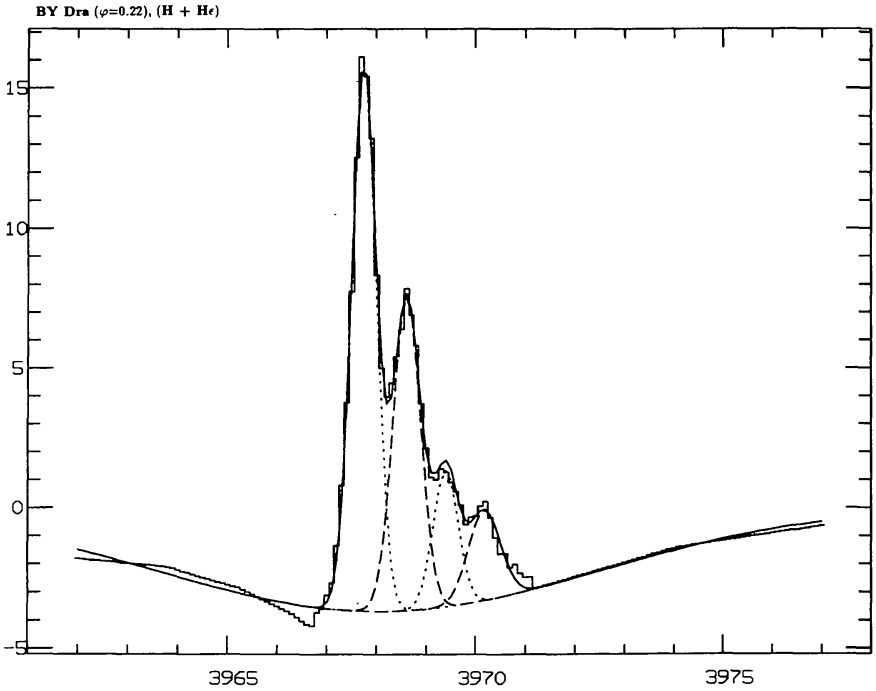

FIG. $3 f$

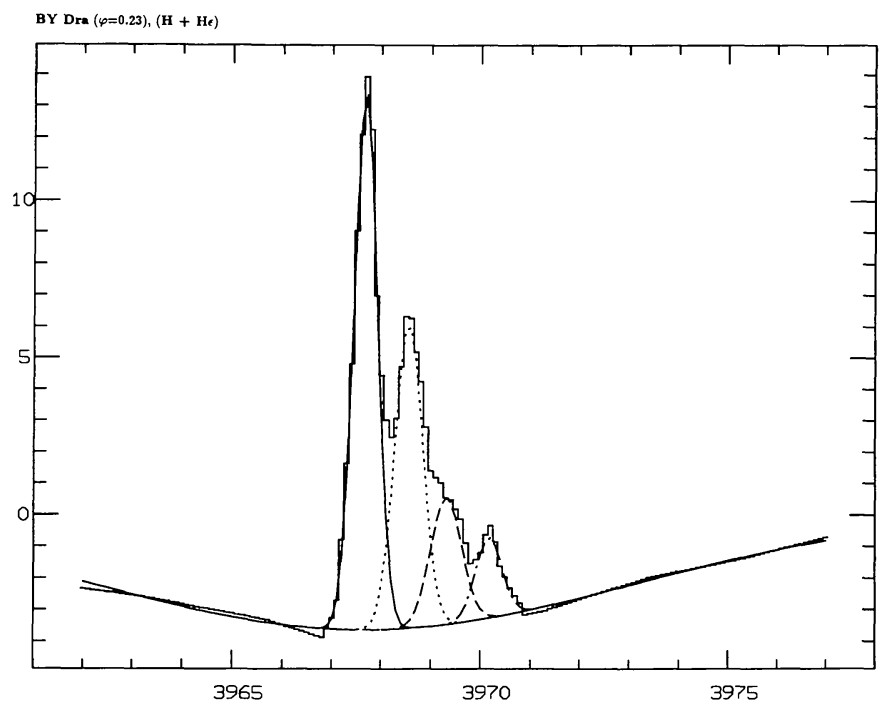

FIG. $3 h$

FIG. 3-Continued

\subsubsection{Z Herculis (HD 163930)}

This system is a spectroscopic binary with partial eclipses. The main contribution to its spectrum comes from the F4 V-lV star; the cool companion (K0 IV) is responsible for the emission. Armentia et al. (1990) found Ca II H and K flux variations with time in comparison with previous observations (Fernández-Figueroa et al. 1986a).

The $\mathrm{H} \alpha$ profile, observed by us at 0.64 phase, is clearly produced by an F4 and a K0 star. The strongest absorption belongs to the hot component. The measured equivalent width of the active component has been corrected by taking into account the contribution of the other component to the total continuum by means of the revised stellar parameters given by Popper (1988). The obtained value points out that the $\mathrm{H} \alpha$ line is not filled in by chromospheric emission. Previously, Weiler
(1978) found a small filling-in of the absorption line but later Bopp \& Talcott (1980) concluded the reverse. The $\mathrm{H} \alpha$ data confirm that $\mathrm{Z}$ Her presents variable chromospheric activity.

\subsubsection{Herculis (HD 341475)}

This system is another double-lined spectroscopic binary. Two spectra were taken in the region of the $\mathrm{Ca}$ II $\mathrm{H}$ and $\mathrm{K}$ lines at orbital phases 0.27 and 0.98 . At phase 0.27 (outside of eclipse) it is possible to see the presence of two emission peaks coming from both components (Fig. 6): the strongest redshifted emission belongs to the cool component and the other, at nearly the center of the absorption, belongs to the hot component. At phase $0.98,30 \%$ of the hot component is in eclipse, and it is not possible deblend the emissions of the two components, so we have measured the total flux in $\mathrm{H}$ and $\mathrm{K}$ emissions (see Table 4). 
KZ And $(\varphi=0.33)$, (K)

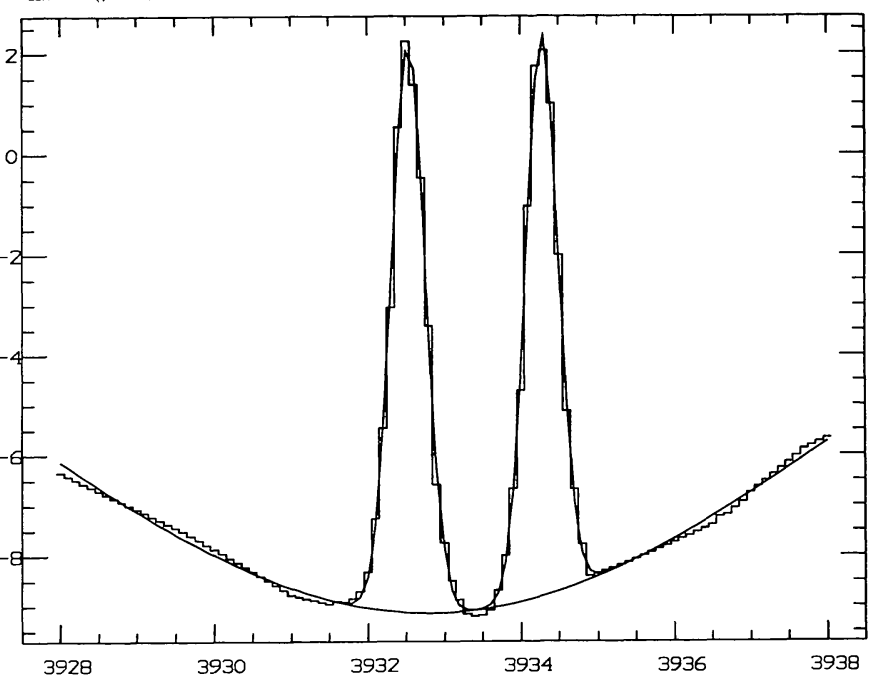

FIG. $4 a$

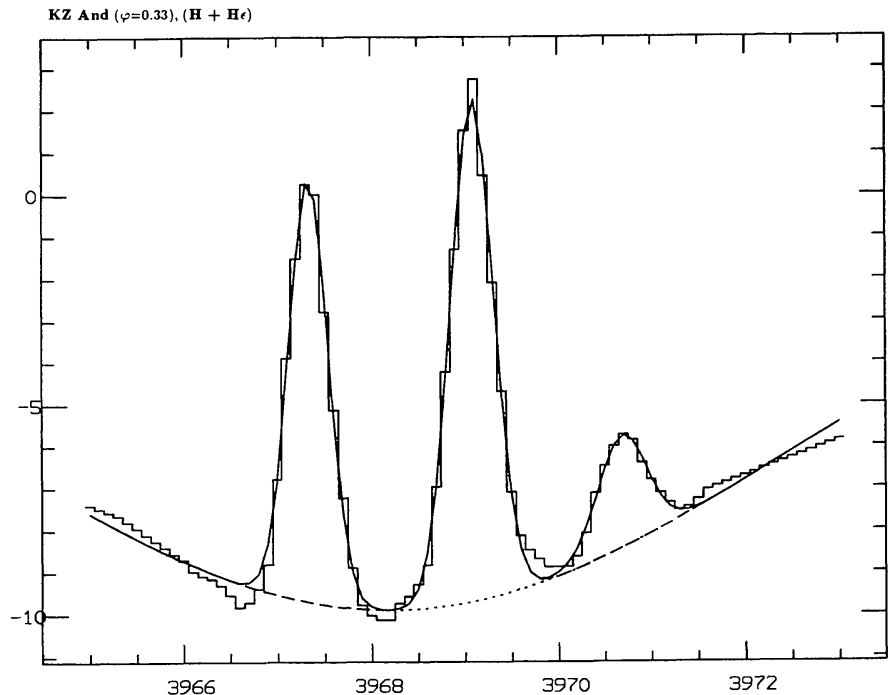

FIG. $4 b$

FIG. 4.-Gaussian fits to the $\mathrm{Ca}$ II $\mathrm{H}$ and $\mathrm{K}$ and the $\mathrm{H} \epsilon$ lines in $\mathrm{KZ}$ And (at 0.33 phase). The dotted and dashed lines are the Gaussian fit and the continue line is the observed spectrum.

One spectrum in the red region was taken in 1989 July at phase 0.24 in which we can see the two $\mathrm{H} \alpha$ lines of the two components as absorption features. According to the orbital phase, the strongest absorption belongs to the cool component, (See Fig. 7). The very different residual intensity of these two absorptions is due to the different contributions of hot and cool components to the continuum $\left(f_{H} / f_{C}=0.43\right)$.

\subsubsection{AW Herculis (HD 348635)}

AW Her is a detached subgiant eclipsing binary discussed by Popper (1990). We present a spectrum at 0.91 phase near to the primary eclipse where the cool component begins to hide the hot component. A strong $\mathrm{H}$ and $\mathrm{K}$ emission, nearly centered in the absorption, can be seen in Figure 6. According to the orbital phase this emission belongs to the cool component.

\subsubsection{Capricorni (HR 8283, HD 206301)}

This system is a single-lined spectroscopic binary classified as G2 IV by Batten, Fletcher, \& Mann (1978), although The Bright Star Catalogue (Hoffleit \& Jaschek 1982) gives the spectral type G1 V/G0 V and notes a single-line spectrum.

One observation at 0.18 phase is shown in Figure 6 , where very weak $\mathrm{Ca}$ II $\mathrm{H}$ and $\mathrm{K}$ emissions centered in the absorption lines can be seen. This behavior indicates that the emission and continuum belong to the component which gives rise to observed spectrum.

\subsubsection{RT Lacertae (HD 209318)}

This system is a G-type eclipsing binary with mass ratio far from unity. Both components of this system present emission in the $\mathrm{H}$ and $\mathrm{K}$ lines. It is normally accepted (CABS) that the cool component is more active but Armentia et al. (1990) found that both components have similar emissions in some epochs.

Two spectra at phases 0.68 and 0.33 in the region of the $\mathrm{H} \alpha$ line clearly show an emission feature above the continuum. The computed phases and the corresponding Doppler shifts indicate that the cool component is responsible for this emission. Huenemoerder \& Barden (1986) found only a weak $\mathrm{H} \alpha$ excess from both components. Therefore red and blue observations point out that the chromospheric activity of RT Lac is highly variable.

\subsubsection{AR Lacertae (HD 210334)}

AR Lac is a typical RS CVn system with strong $\mathrm{Ca}$ II $\mathrm{H}$ and $\mathrm{K}$ emissions from both components (CABS). We have carried out two observational campaigns, in the first one (1985) the emissions from both components were clearly visible, with the stronger one belonging to the cool star (Montesinos 1986). Unfortunately in the second observational season (1989) we took two spectra at an identical phase $(0.95)$ and it was not possible to separate the contribution from each component due to the orbital phase (Fig. 6). In Table 4 the $H$ and $K$ emission fluxes listed correspond to the sum of the two unresolved lines.

One spectrum centered at the $\mathrm{H} \alpha$ line and at 0.40 phase (Fig. 7) is available. This spectrum shows no definite emission from either component. Again, because of the orbital phase it is not possible to separate the absorption feature corresponding to each component and to find a possible filled-in absorption.

\subsubsection{SZ Piscium (HD 219113)}

This system is a partially eclipsing binary. Three new observations at different epochs and at phases $0.42,0.57$, and 0.32 are presented in order to continue the Ca II monitoring of this 


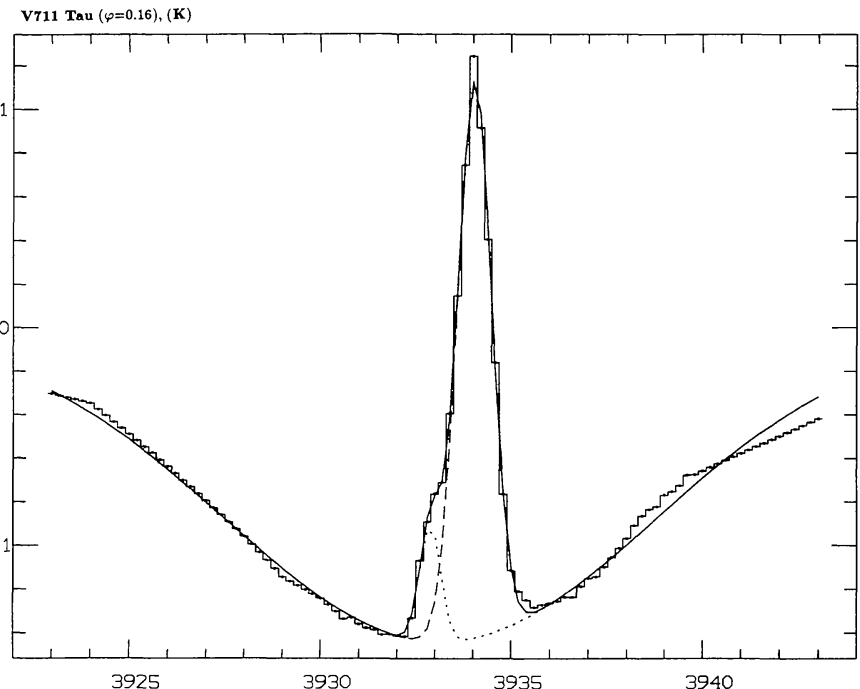

FIG. $5 a$

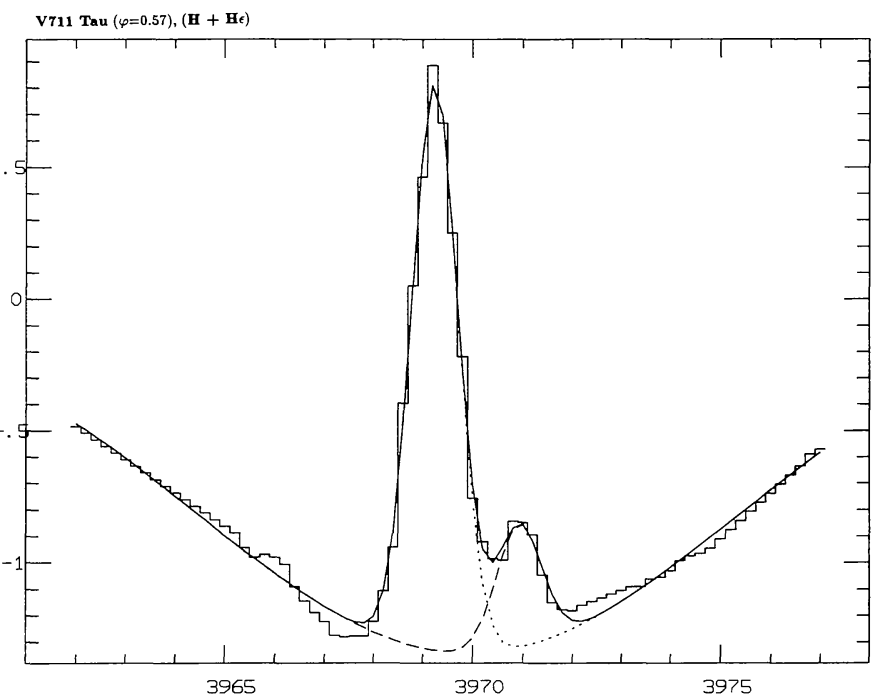

FIG. $5 c$

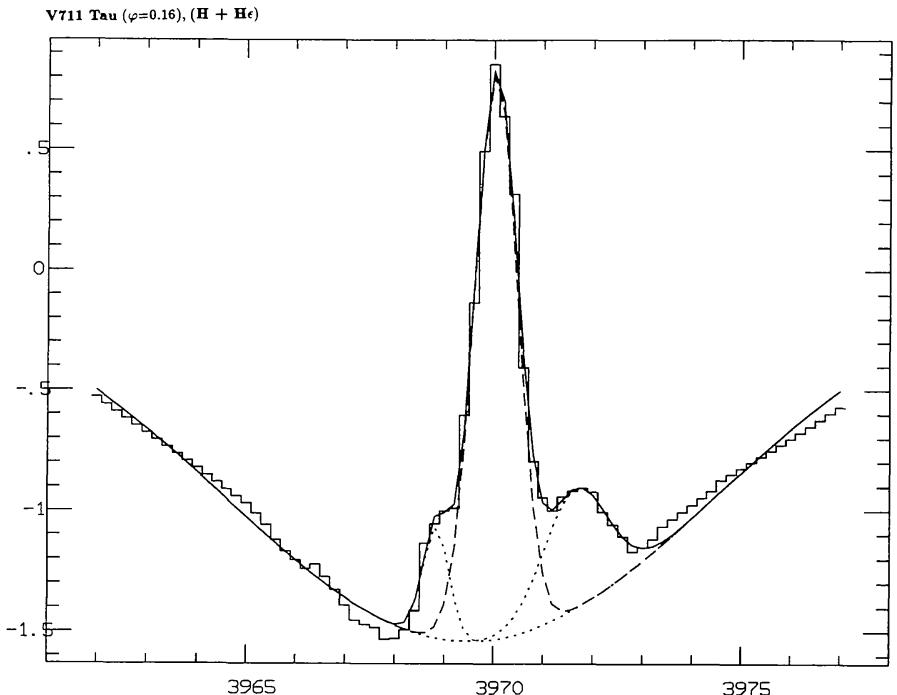

FIG. $5 b$

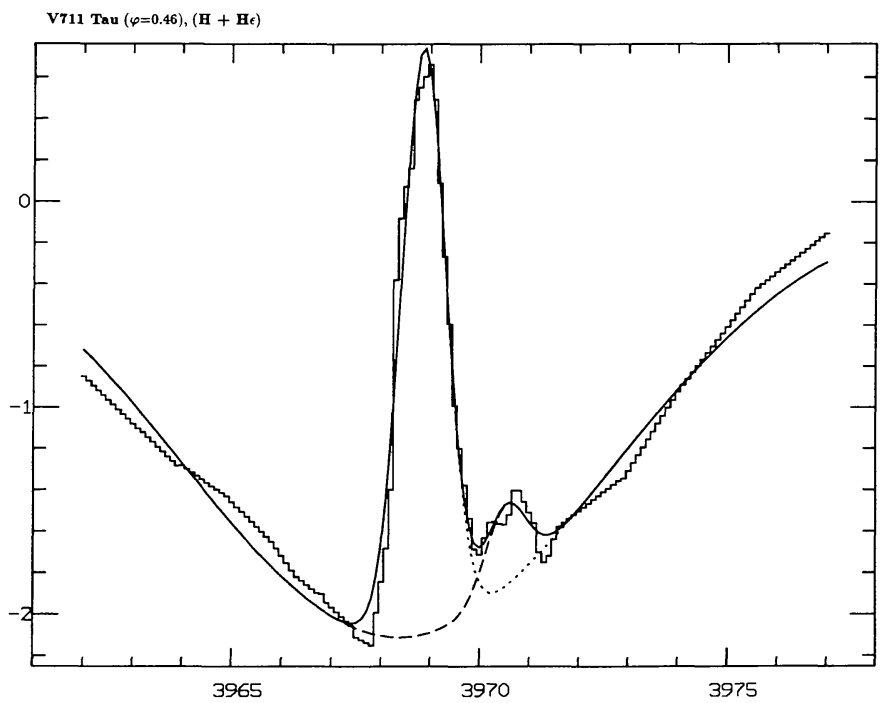

FIG. $5 d$

FIG. 5.-Gaussian fits to the $\mathrm{Ca} I \mathrm{H}$ and $\mathrm{K}$ and the $\mathrm{H} \epsilon$ lines in V711 Tau (at phases $0.16,0.57$, and 0.46). The dotted line is the Gaussian fit and the continuous line is the observed spectrum.

star started in 1985 in which the appearance of a transient micro flarelike phenomenon was noticed (Fernández-Figueroa et al. 1986b). The new $\mathrm{Ca}$ II $\mathrm{H}$ and $\mathrm{K}$ emission fluxes are given in Table 4. The flux variations are of the same order as those obtained in 1985 .

Our spectrum centered at $\mathrm{H} \alpha$ region, taken at a phase close to the quadrature, exhibits two $\mathrm{H} \alpha$ absorptions, the strongest one coming from the cool component (see Fig. 6). The measured equivalent widths of both component have been corrected taking into account that the ratio between the continuum flux of both components is 0.39 . From these values we conclude that the cool component presents a filled-in absorption line profile.

\subsection{Group 3 (Active Component of Luminosity Class III)}

\subsubsection{Piscium (HD 28)}

The 33 Psc system has been classified as an RS CVn star with no photometric spot wave (CABS). Our spectra at phase 0.19 exhibit quite weak $\mathrm{Ca}$ II $\mathrm{H}$ and $\mathrm{K}$ emissions (Fig. 6). The value of the parameter $C(\mathrm{~K})$ is the smallest in the group which agrees with the Wilson classification $\left(I_{K}=2\right)$ (Wilson 1976). Our $\mathrm{H} \alpha$ observation confirms the extremely weak chromospheric activity of this system since the $\mathrm{H} \alpha$ line appears as a strong absorption feature. 

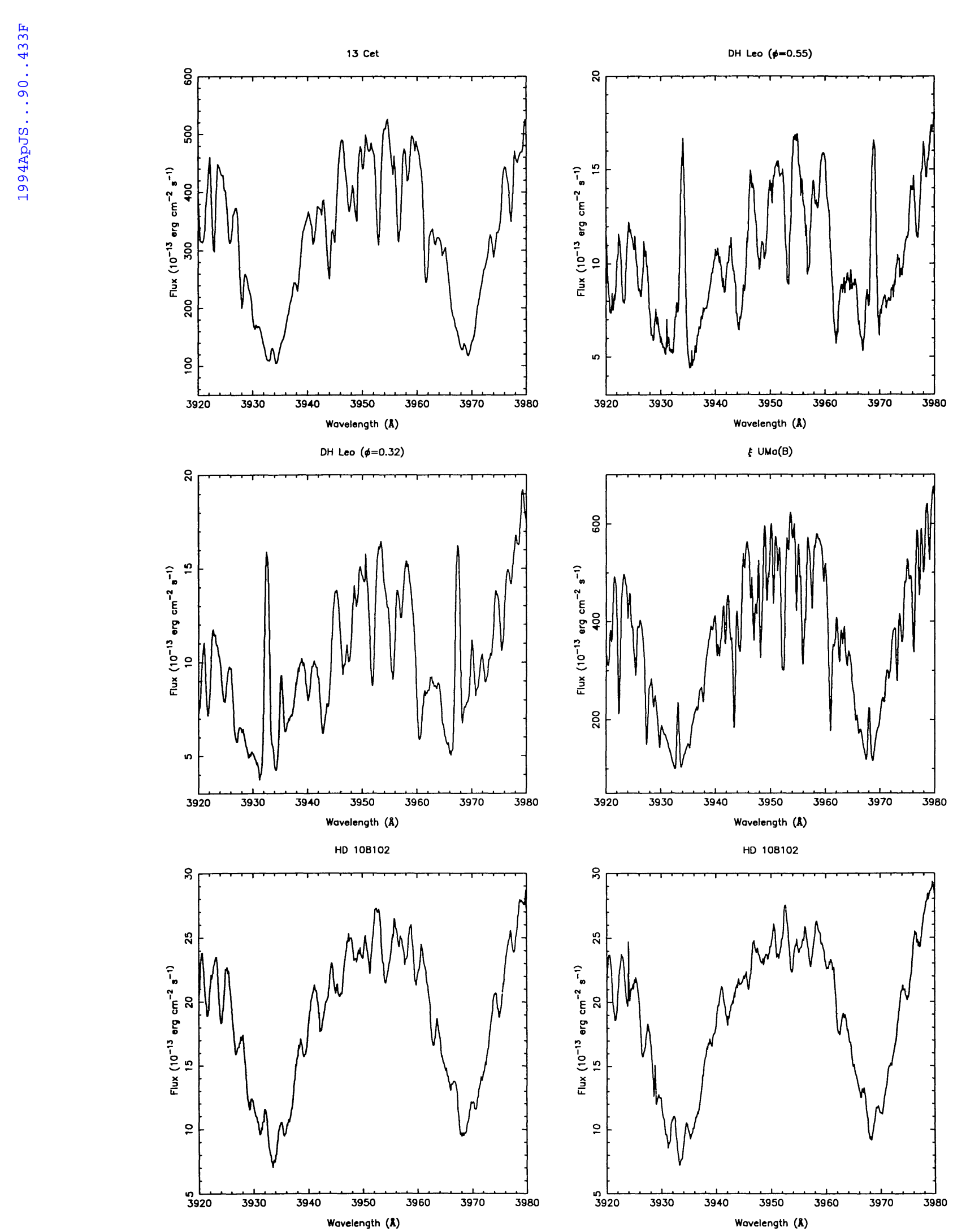

FIG. 6.-Ca II H and $\mathrm{K}$ spectra between 3920 and $3980 \AA \AA$. Fluxes are in units of $10^{-13} \mathrm{ergs}^{-2} \mathrm{~s}^{-1} \AA^{-1}$. In each plot the name of the star is indicated 

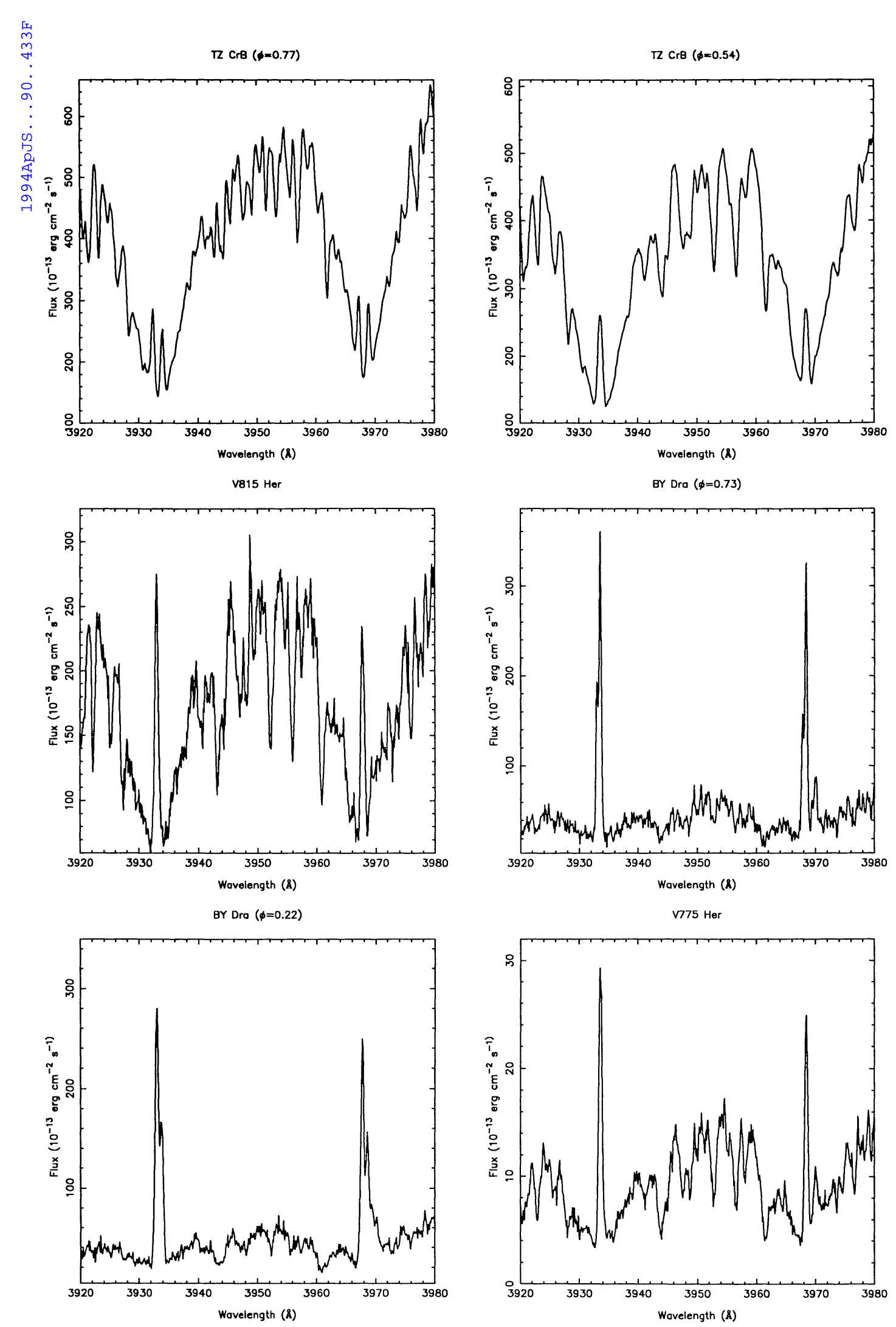

FIG. 6-Continued 

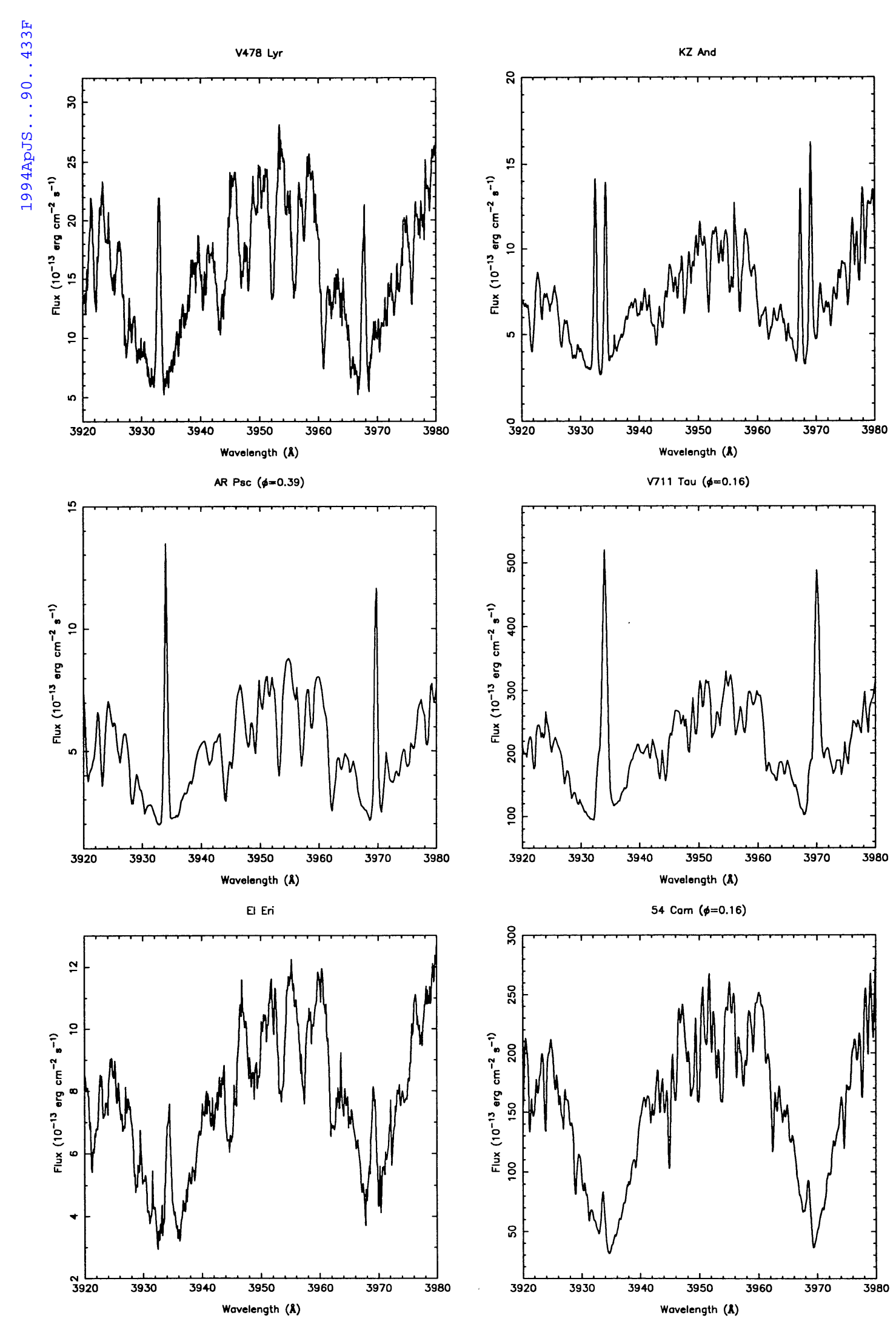

FIG. 6-Continued 

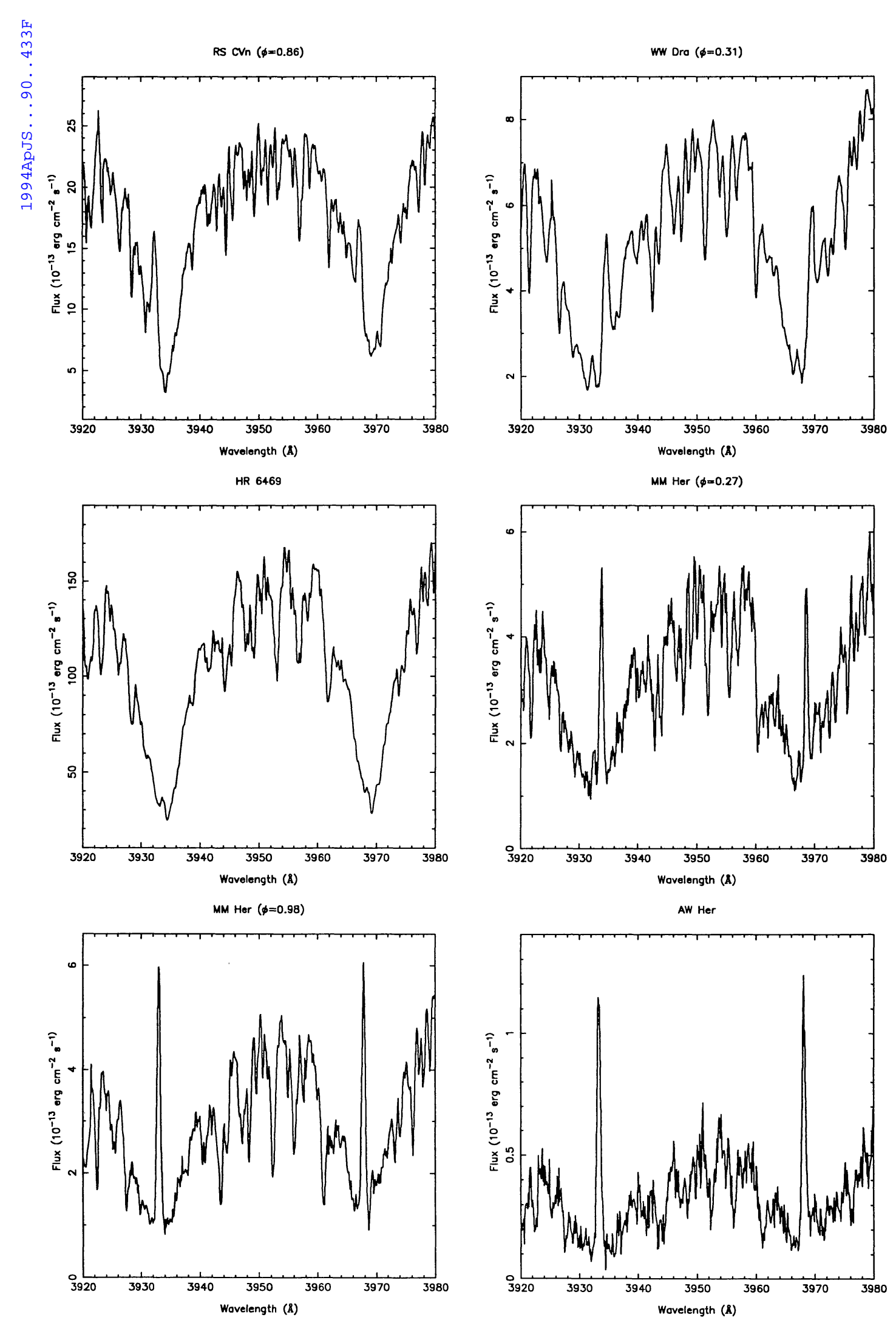

FIG. 6-Continued 

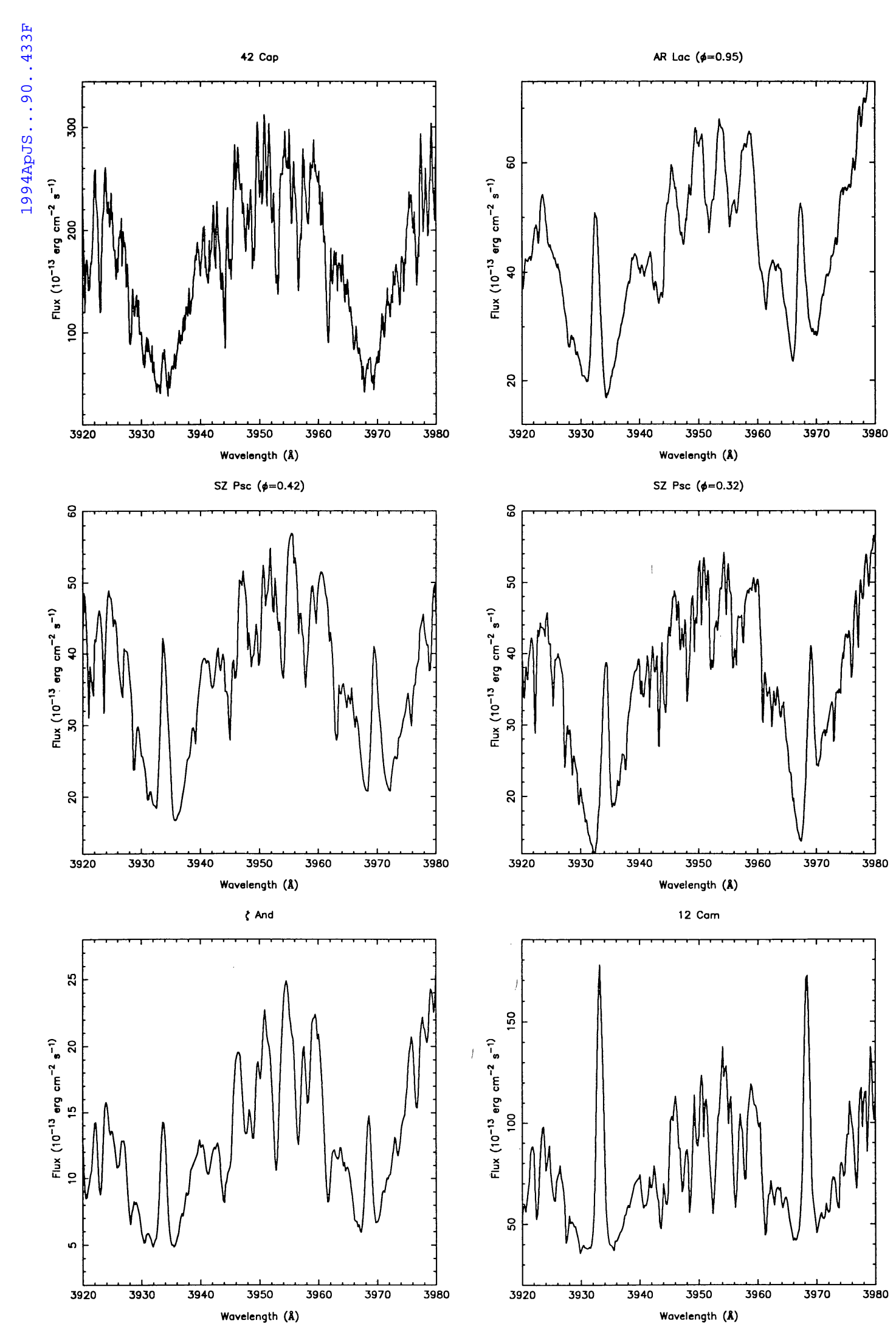

Fig. 6-Continued 
PLATE 1

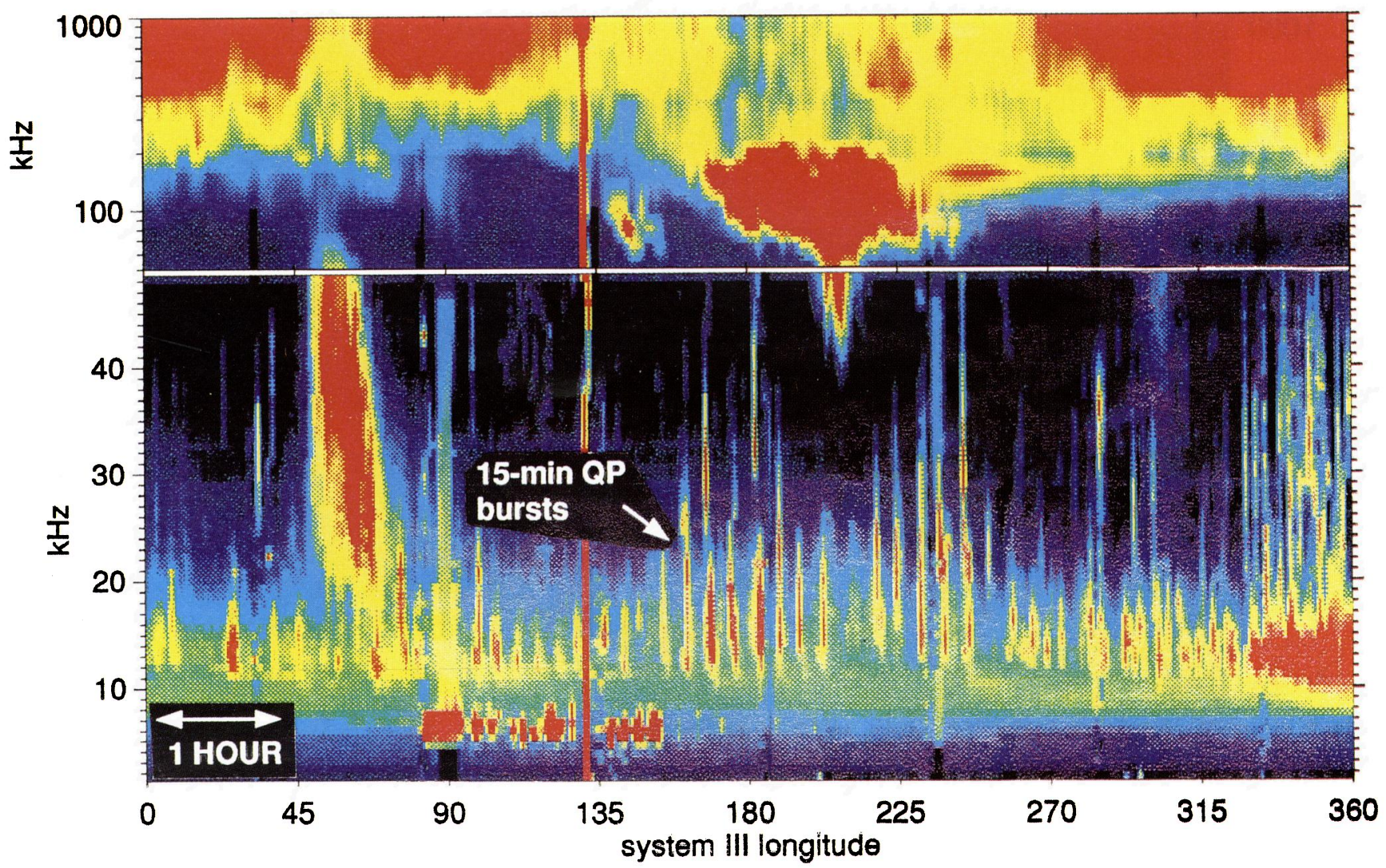

FIG. 1.-Frequency-time radio spectrogram beginning on 1992 February 1 from the Ulysses URAP experiment showing quasi-periodic (QP) bursts. The QP bursts are visible as vertical structures in the spectrogram extending from about 10 to $50 \mathrm{kHz}$ for almost the entire day. 

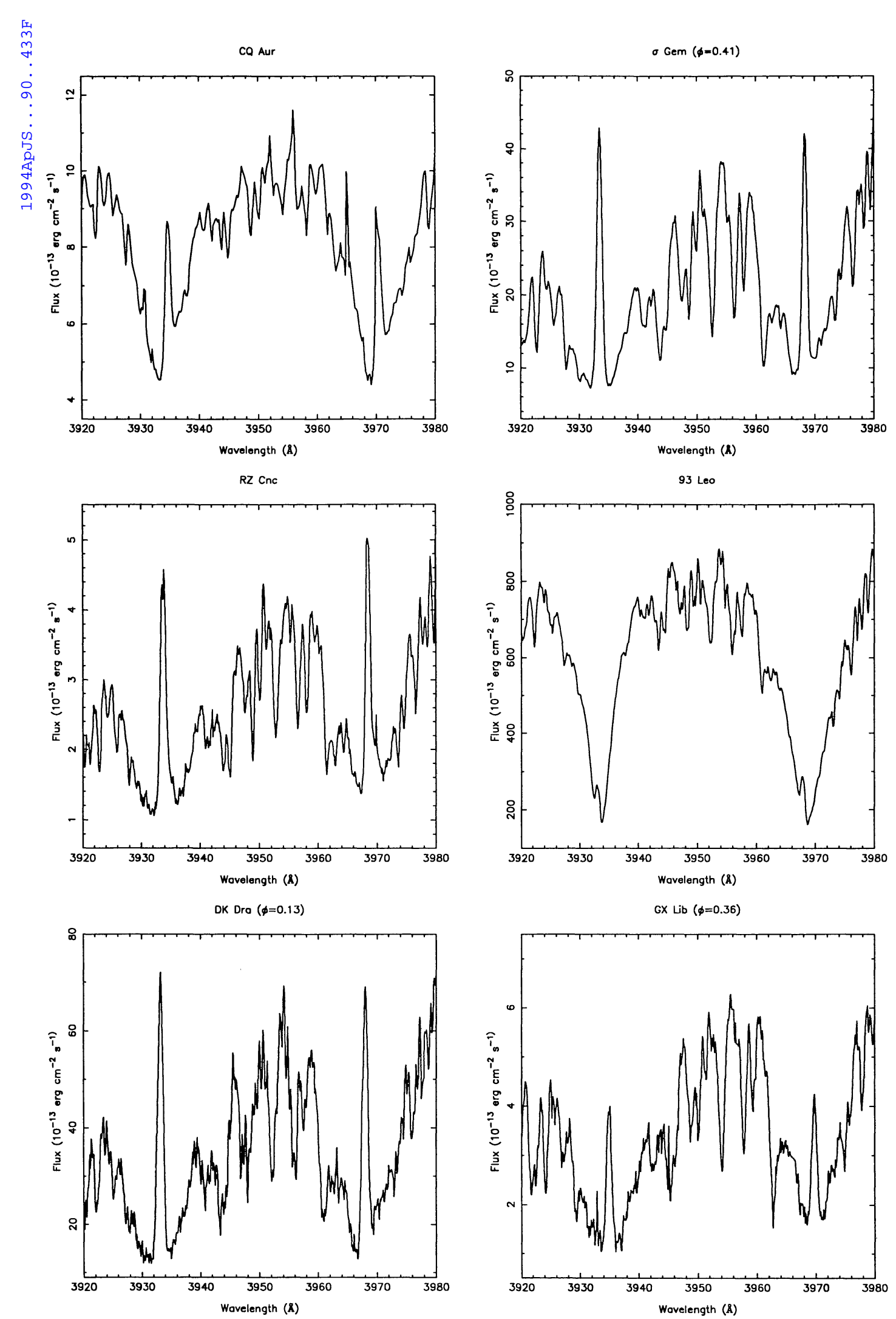

FIG. 6-Continued 
PLATE 2

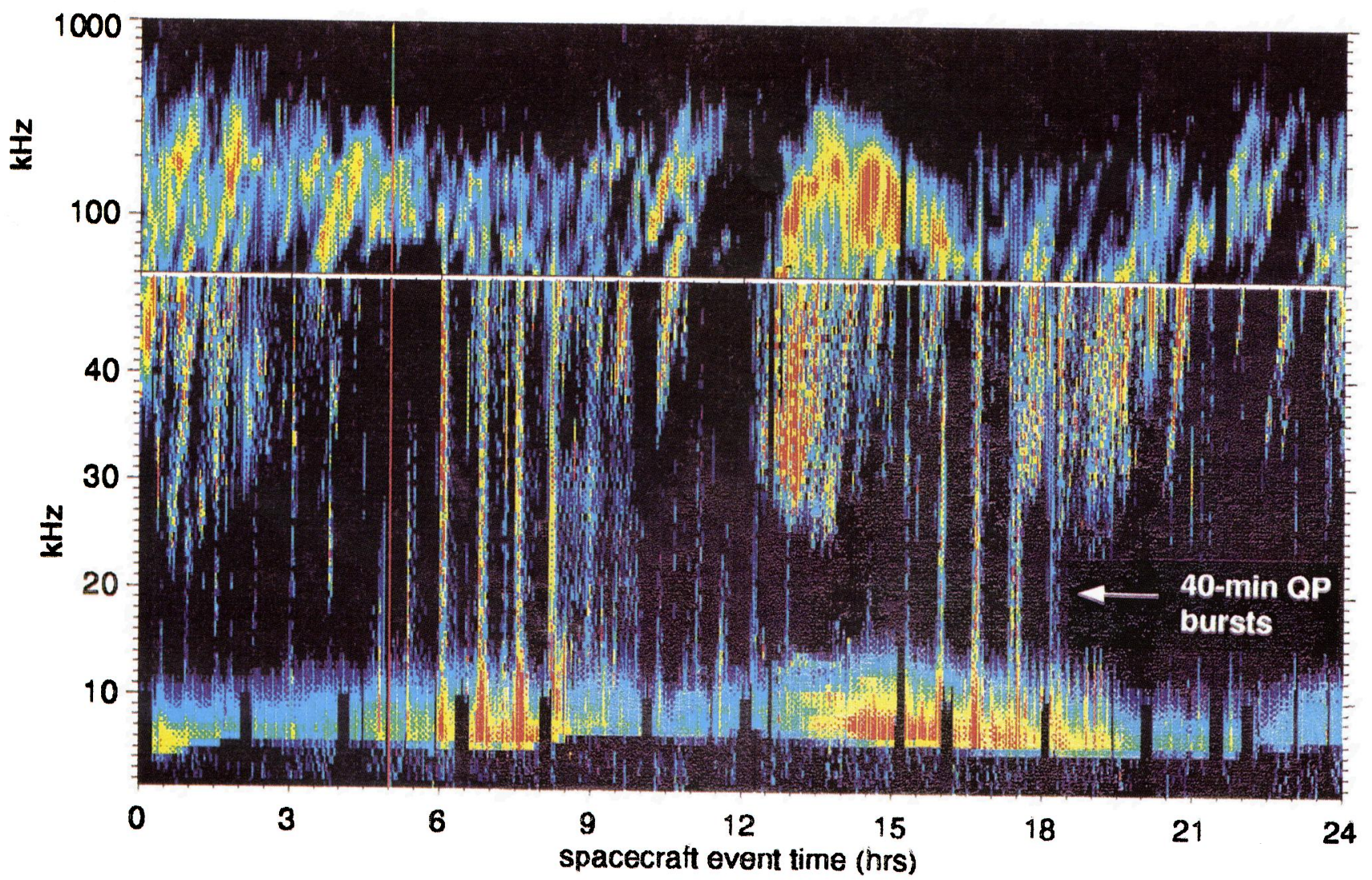

FIG. 6.- Outbound observations by the Ulysses URAP experiment of Jovian QP bursts on 199215 February. The bursts are most prominent between about 5 and $50 \mathrm{kHz}$ and from 6 to $9 \mathrm{hr}$ and 16 to $18 \mathrm{hr}$ spacecraft event time. The observed periodicity is about 40 minutes. 

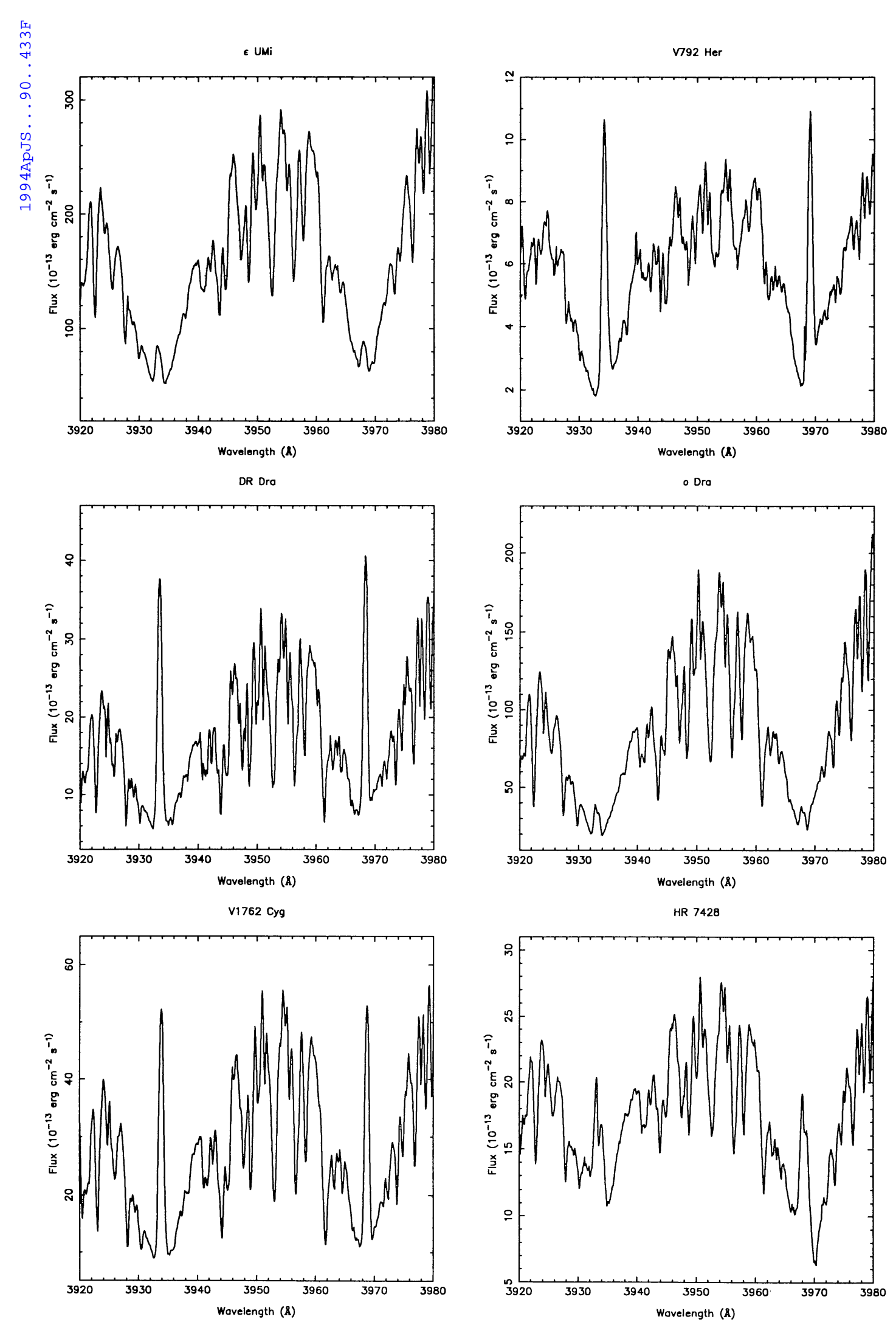

FIG. 6-Continued 

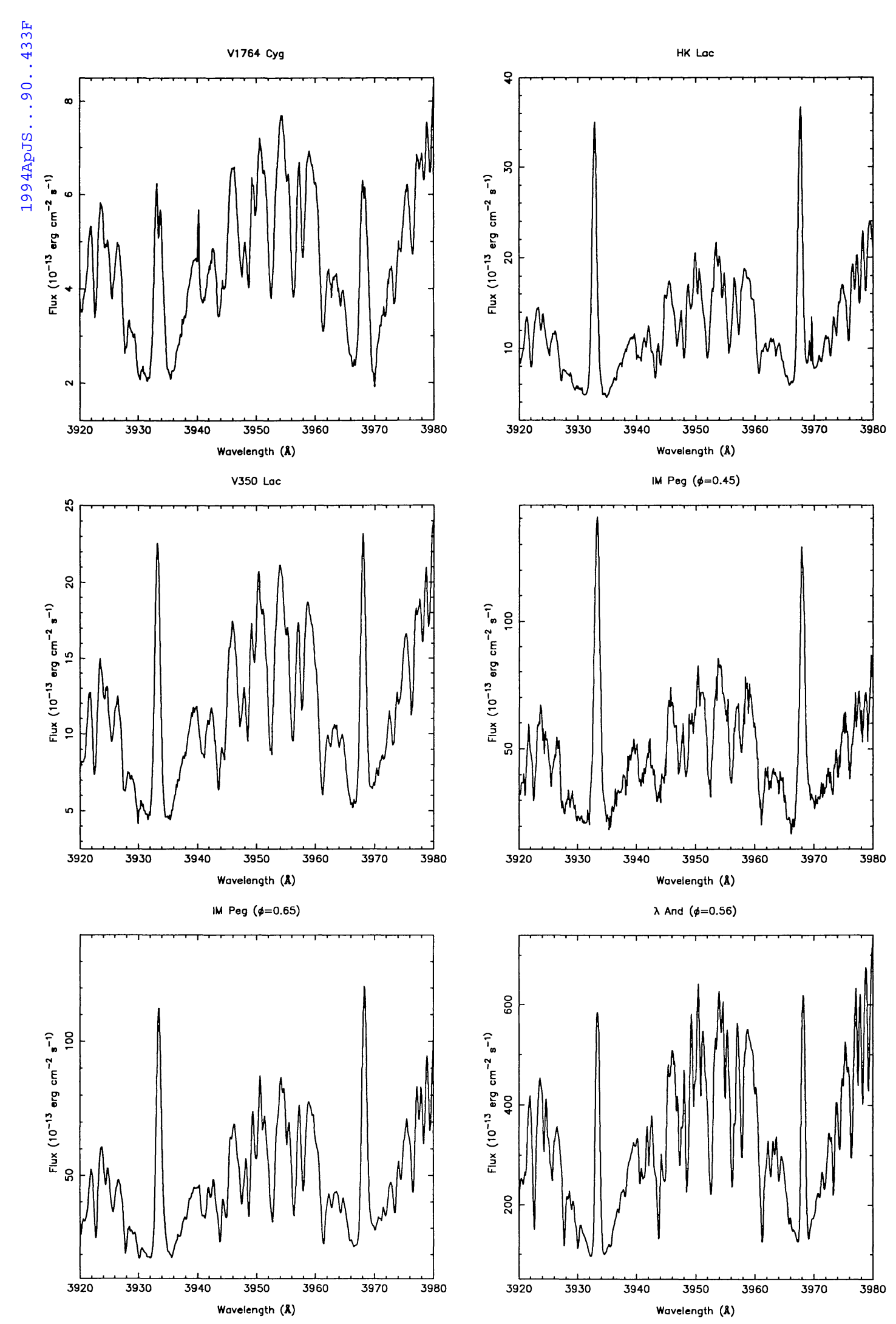

Fig. 6-Continued 

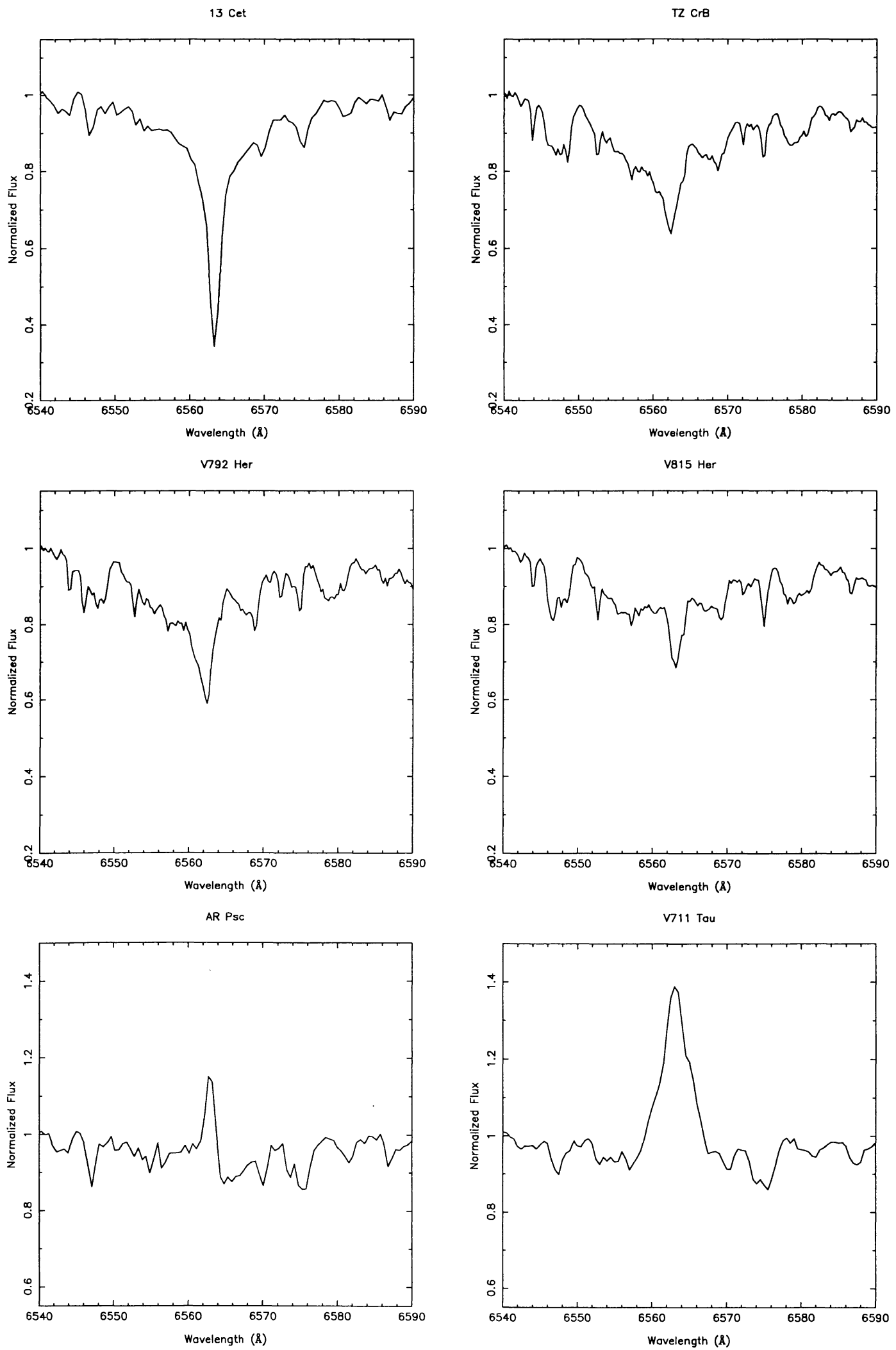

FIG. 7.- $\mathrm{H} \alpha$ spectra between 6540 and $6590 \AA$. Fluxes have been normalized, and in each plot the name of the star is indicated 

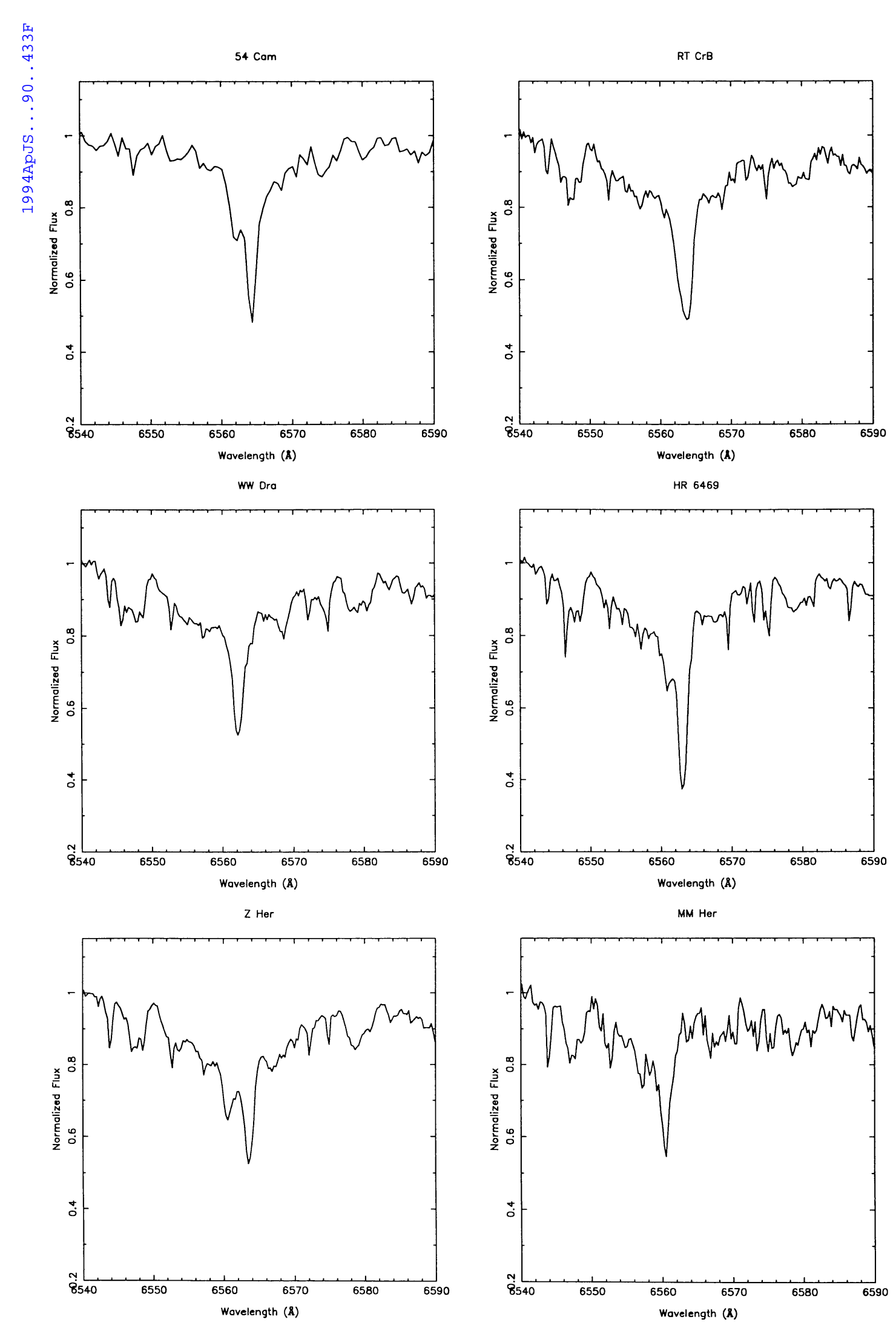

Fig. 7-Continued 

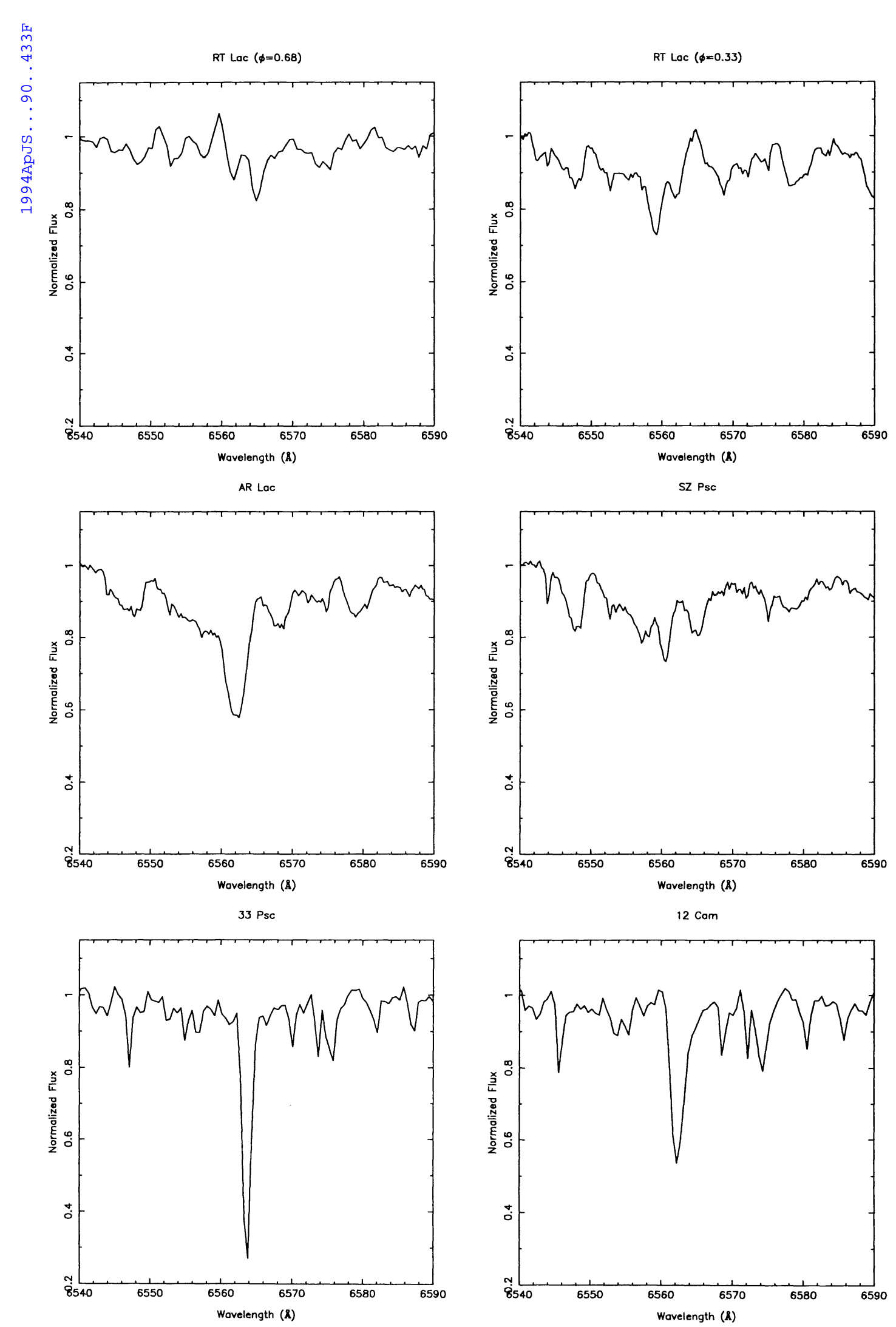

Fig. 7-Continued 

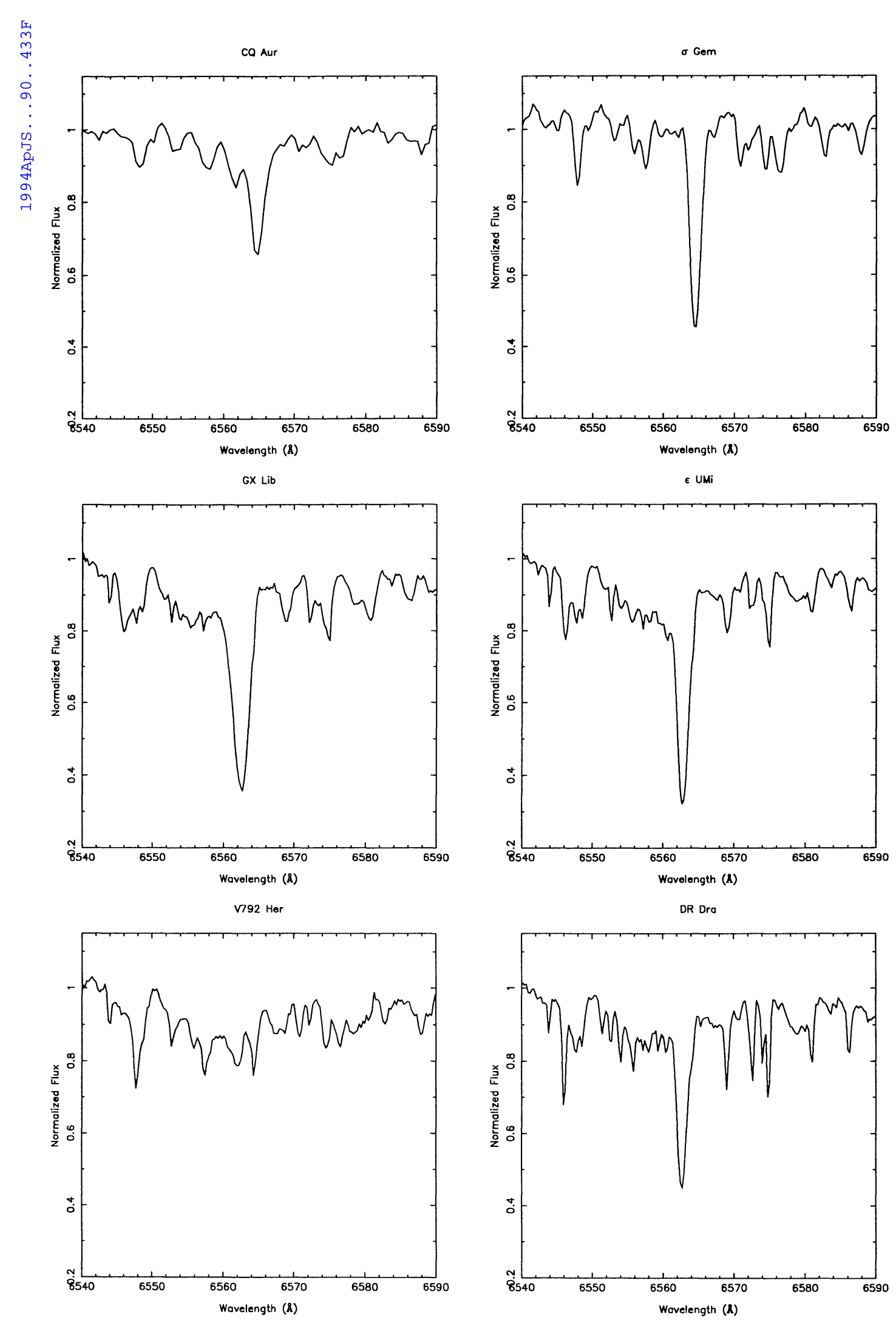

FIG. 7-Continued 
HK Loc
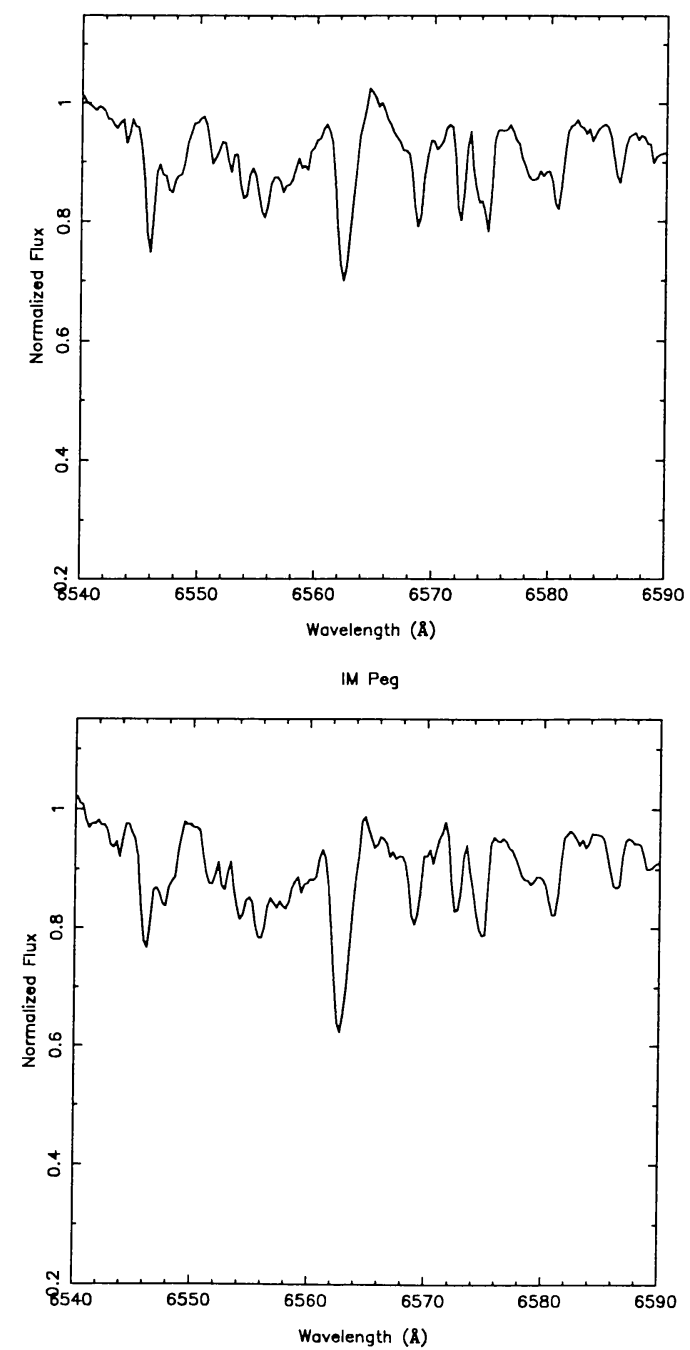

V350 LoC
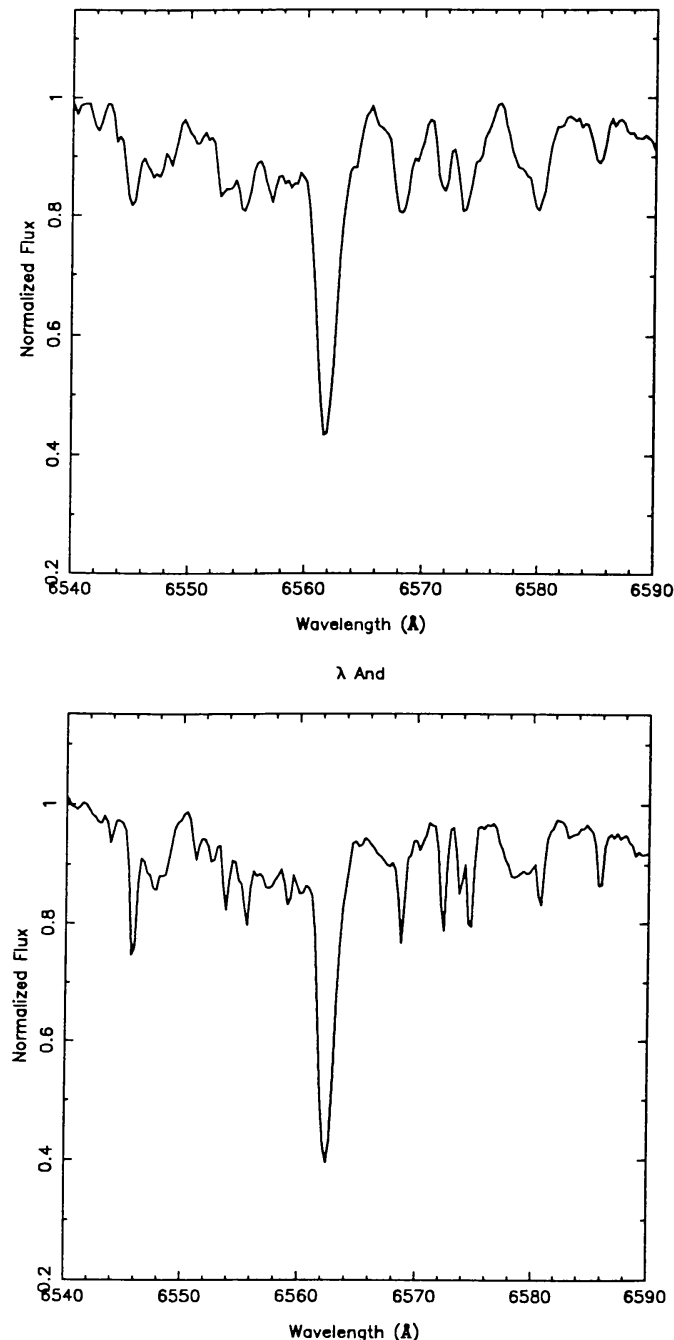

Fig. 7-Continued

\subsection{2. $\zeta$ Andromedae ( 34 Andromedae, HD 4502)}

This system is classified as K1 II in CABS but the radius calculated by Strassmeier et al. (1989) is inconsistent with its luminosity classification. Here we present a spectrum of the $\mathrm{Ca}$ II $\mathrm{H}$ and $\mathrm{K}$ lines at 0.29 phase where a strong emission centered at the absorption can be seen. Another observation, in the $\mathrm{H} \alpha$ region at 0.85 phase, shows a strong absorption.

\subsubsection{Camelopardalis (BM Camelopardalis, HD 32357)}

Four observations of the $\mathrm{Ca}$ II $\mathrm{H}$ and $\mathrm{K}$ lines show that this K0 III system exhibits a strong emission centered at the absorption line. The $\mathrm{H} \alpha$ line profile of this system shows a strong absorption feature. The equivalent width and residual intensity listed in Table 5 point out that the line is only slightly filled-in.

\subsubsection{CQ Aurigae (HD 250810)}

CQ Aur is a detached eclipsing binary with $\mathrm{Ca}$ II $\mathrm{H}$ and $\mathrm{K}$ emissions from the cooler component and has recently been reviewed by Popper (1990). Two observations in the region of the $\mathrm{Ca}$ II $\mathrm{H}$ and $\mathrm{K}$ lines at orbital phases 0.42 and 0.95 are available. At 0.42 phase a redshifted emission is clear, and at 0.95 phase the emission is near the center of the absorption line. This behavior confirms that the emission belongs to the cool component.

In our observation at 0.42 phase in the $\mathrm{H} \alpha$ region a weak absorption feature can be seen (Fig. 7). The measured equivalent width indicates that the line is filled-in by chromospheric emission.

\subsection{5. $\sigma$ Geminorum ( 75 Geminorum, HD 62044)}

This single-lined spectroscopic binary system belongs to the long-period group of RS CVn binary systems. Three $\mathrm{Ca}$ II $\mathrm{H}$ and K spectra were taken in 1988 and one more in 1991. At every phase the emission is centered at the absorption line. The measured surface fluxes of the $\mathrm{H}$ and $\mathrm{K}$ lines are comparable to those computed by Bopp (1983), within the errors. The flux of the 1991 observation is higher than those corresponding to 1988; these variations are consistent with active-area changes 
associated with a 2.7 yr starspot cycle (Strassmeier et al. 1988). One observation at 0.13 phase in the $\mathrm{H} \alpha$ region shows a strong absorption.

\subsubsection{RZ Cancri (HD 73743)}

This spectroscopic binary is formed by a pair of giants ( $\mathrm{K} 1$ III/K3-4 III), with the cooler component filling its Roche lobe. One observation of $\mathrm{Ca}$ II $\mathrm{H}$ and $\mathrm{K}$ lines at 0.36 phase shows (Fig. 6) strong emission in the center of the absorption line, from which we infer that the hot component is the responsible for this emission.

\subsubsection{Leonis (DQ Leonis, HD 102509)}

This is a spectroscopic binary system, in which Young \& Koniges (1977) reported the presence of the $\mathrm{Ca}$ II $\mathrm{H}$ and $\mathrm{K}$ emission as moderately strong emission features. Later, Batten et al. (1983) found no obvious emissions in the $\mathrm{H}$ and $\mathrm{K}$ lines.

Our two spectra taken in 1988 (Fig. 6) show that if any actual emission is present, it is very weak. According to the calculated orbital phases $(0.43$ and 0.46$)$ the emission should be redshifted, whereas we observed the opposite shift. The weak observed peak could also be explained as a small division between the absorptions of the two components, in this case the wavelength difference between the two absorptions does not correspond either to the computed radial velocity. Finally, we conclude that the presence of $\mathrm{H}$ and $\mathrm{K}$ emissions in $93 \mathrm{Leo}$ is "not obvious," as Batten et al. (1983) mentioned.

\subsubsection{DK Draconis (HD 106677)}

This is a double-lined spectroscopic binary with almost identical components of spectral type K0 III (Bopp et al. 1979; Fekel et al. 1986). Our Ca II H and K spectroscopic observations cover two observing seasons in 1986 and in 1988, although only spectra at phases 0.44 and 0.1 are available. We observe a strong emission centered at the absorption but, due to the orbital phases, it is not possible to establish whether the emission comes from both component or from only one.

\subsubsection{GX Librae (HD 136905)}

This single-lined spectroscopic binary shows $\mathrm{H}$ and $\mathrm{K}$ emission lines of moderate strength (Fekel et al. 1986). We present two observations of the $\mathrm{Ca}$ II $\mathrm{H}$ and $\mathrm{K}$ lines at phases 0.44 and 0.36 , which show a wide emission line at the center of the absorption line. In the red spectrum, taken at 0.80 phase, the $\mathrm{H} \alpha$ line exhibits a strong absorption feature.

\subsubsection{0. $\epsilon$ Ursae Minoris (22 Ursae Minoris, HD 153751)}

The primary component ( $\epsilon \mathrm{UMi}$ ) of the visual pair (ADS 10152 ) is a binary system with grazing-total eclipse (Hinderer 1957). We present a spectrum of $\epsilon \mathrm{UMi}$ at 0.43 phase in the region of the $\mathrm{Ca}$ II $\mathrm{H}$ and $\mathrm{K}$ lines. A moderate emission centered at the absorption line can be seen in Figure 6. Taking into account the high luminosity of the cool component (G5 III) we can infer that the observed spectrum, as well as the $\mathrm{H}$ and $\mathrm{K}$ emissions, comes from this cool component. In addition, one observation of the $\mathrm{H} \alpha$ line at 0.53 phase revealed that the line shows a filled-in absorption line profile.

\subsubsection{V792 Herculis (HD 155638)}

V792 Her is an eclipsing and double-lined RS CVn binary system. Only one observation at 0.10 phase covering the $\mathrm{Ca}$ II $H$ and $K$ region is available (Fig. 6). The $H$ and $K$ lines are seen as redshifted strong emissions, in agreement with the orbital velocity of the cool component.

Two absorptions are present in our $\mathrm{H} \alpha$ observation at 0.21 phase. The strongest absorption belongs to the cool component. The measured equivalent widths of both components have been corrected taking into account that the ratio between the continuum flux of both components is 0.27 . These values allow us to conclude that the cool component presents a filledin absorption line profile.

\subsubsection{DR Draconis (29 Draconis, HD 160538)}

DR Dra is a single-lined spectroscopic binary formed by a hot white dwarf and a K0-2III primary (Fekel \& Simon 1985) in addition, this system is a highly asynchronous rotator.

Here we present one observation in the $\mathrm{Ca}$ II $\mathrm{H}$ and $\mathrm{K}$ region, taken in 1989 July, showing strong $\mathrm{H}$ and $\mathrm{K}$ emissions above the continuum. One spectrum of the $\mathrm{H} \alpha$ line, taken in 1989 July, shows a weak filled-in absorption feature.

\subsubsection{3. o Draconis (47 Draconis, HR 7125, HD 175306)}

o Dra is a SB1 binary classified as G9 III by Keenan \& Pitts (1980). We have only one spectrum of this system in the Ca II $\mathrm{H}$ and $\mathrm{K}$ region taken at 0.66 phase which presents a weak $H$ and $\mathrm{K}$ emission (Fig. 6). This observation is in disagreement with the strong $\mathrm{Ca}$ II $\mathrm{H}$ and $\mathrm{K}$ emission reported by Hossak (1954).

\subsubsection{V1762 Cygni (HR 7275, HD 179094)}

This system is a single-lined binary with a quite large variable spot activity (Strassmeier et al. 1989). Our spectroscopic observation in the region of the $\mathrm{Ca}$ II $\mathrm{H}$ and $\mathrm{K}$ lines at 0.45 phase shows a strong emission centered at the absorption line. Our surface flux measurements are comparable to those computed by Bopp (1984), taking into account the different calibration method in absolute flux.

\subsubsection{V1817 Cygni (HR 7428, HD 184398)}

This system, classified as A0 V/K2 III-II (Levato 1975), has a mean photometric period of 54.6 days, nearly half of the orbital period (Strassmeier et al. 1989).

Three observations in two different epochs (1988 and 1989 ), corresponding to phases $0.25,0.27$ and 0.52 , are available. The spectra show the $\mathrm{Ca}$ II $\mathrm{H}$ and $\mathrm{K}$ emission lines not exactly centered at the absorption, due to the contribution of the hot component to the observed spectrum. The emissions seem to present self-absorptions (Fig. 6), probably due to the extended atmosphere around the cool component. In Table 5, only the values corresponding to the highest quality image (1989) are given.

\subsubsection{V1764 Cygni (HD 185151)}

This is a single-lined spectroscopic binary (K1 III) with strong $\mathrm{Ca}$ II $\mathrm{H}$ and $\mathrm{K}$ emission lines (Bopp et al. 1982). 


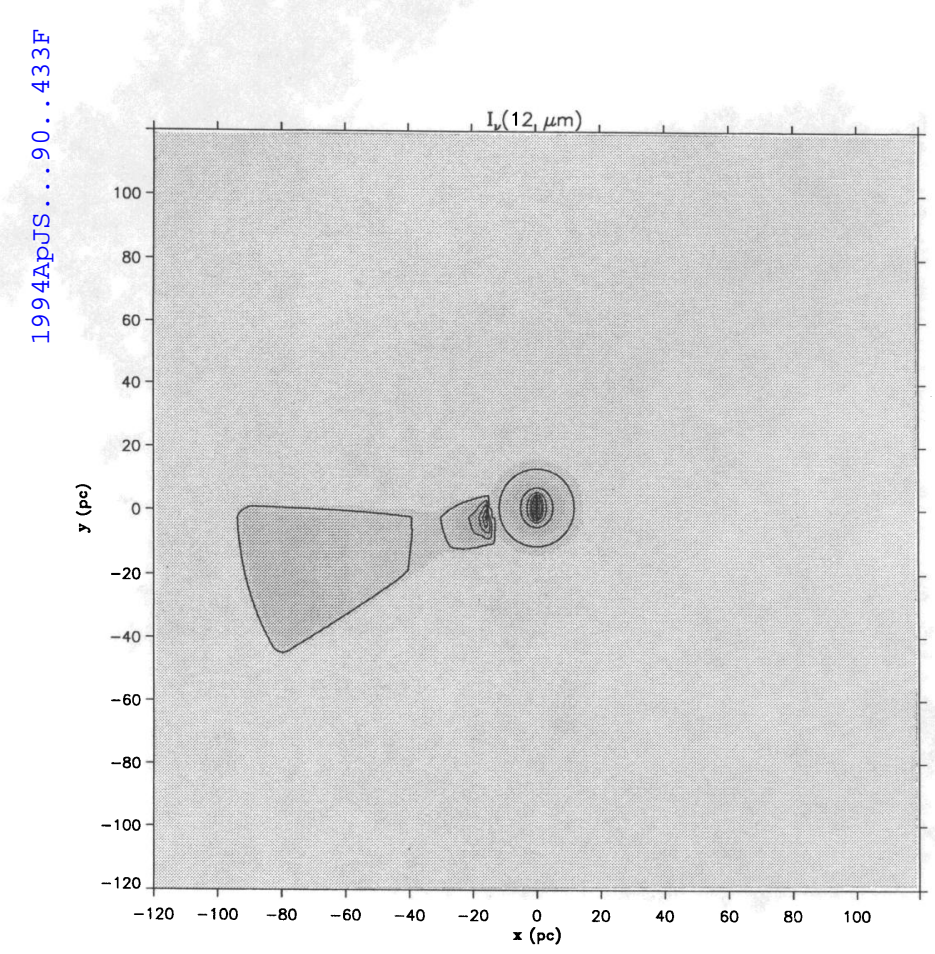

FIG. $10 a$

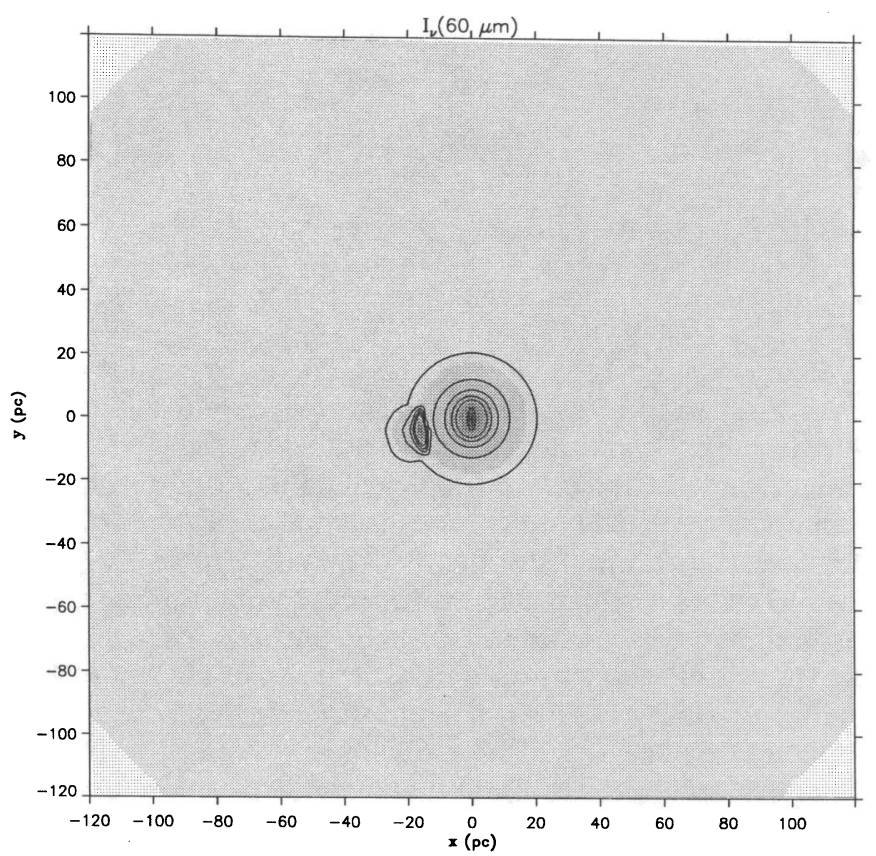

FIG. $10 c$

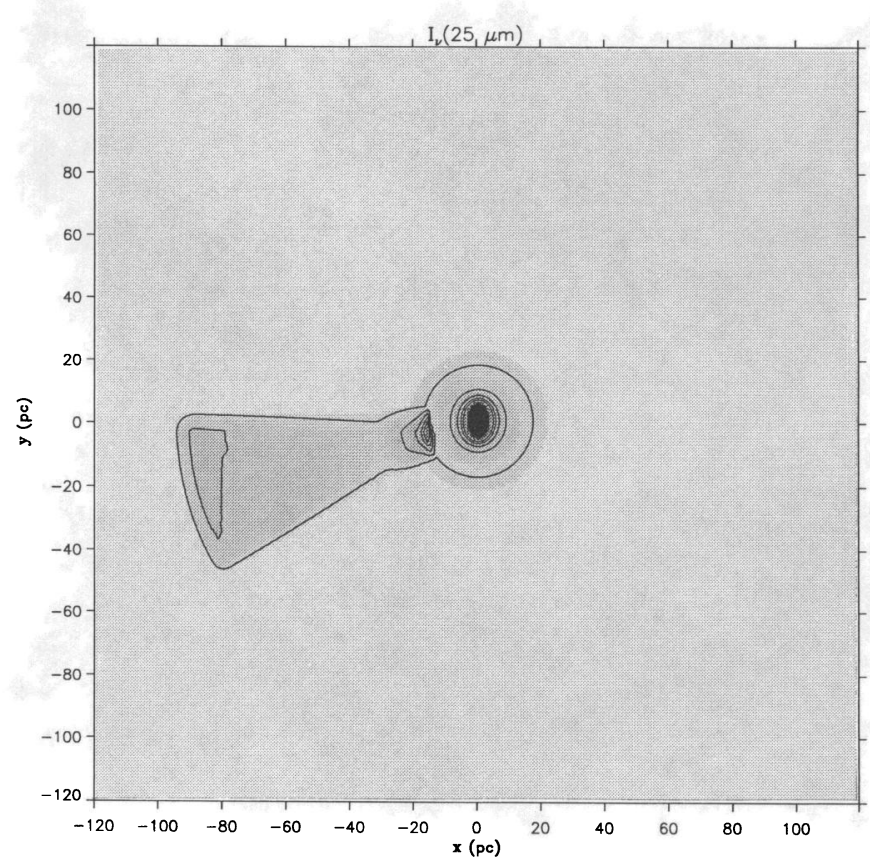

FIG. $10 b$

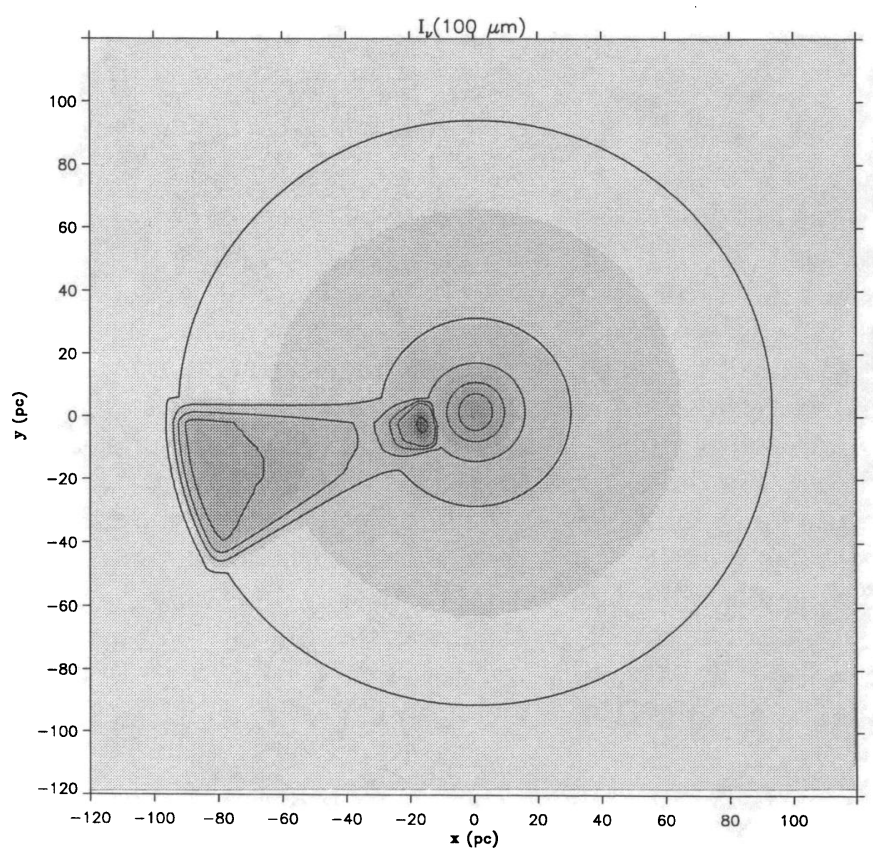

FIG. $10 d$

FIG. 10.-Model surface brightness distributions for cloud orientation case 1 at $(a) 12,(b) 25,(c) 60$, and $(d) 100 \mu \mathrm{m}$ after convolution with the $I R A S$ spatial resolution (see $\S 4$ ). A $120 \times 120$ pc region, centered on the star cluster, is shown. Most of the cloud cannot be seen in Fig. $10 \mathrm{c}$ because the cloud is not much brighter than the $\mathrm{H}$ Il region. In Figs. $10 a$ and $10 b$, contour levels are $1,2,3,4,5$, and $10 \mathrm{MJy} \mathrm{sr}^{-1}$; the levels in Figs. $10 c$ and $10 d$ are $10,20,30,40,50$, 100 , and $200 \mathrm{MJy} \mathrm{sr}^{-1}$. 


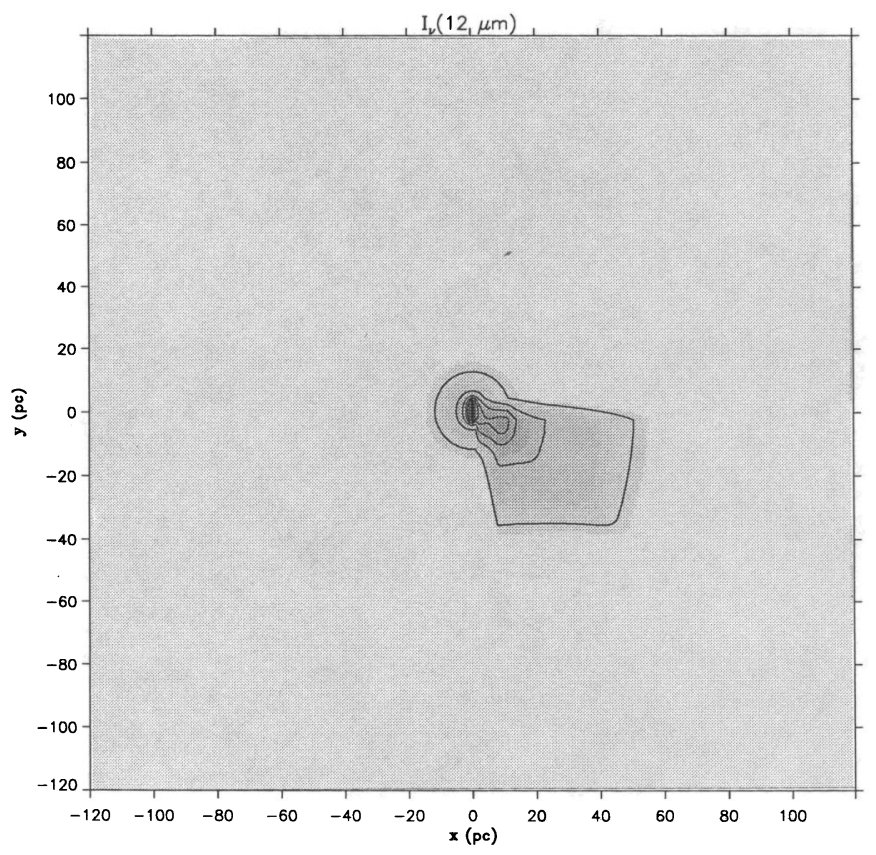

FIG. $11 a$

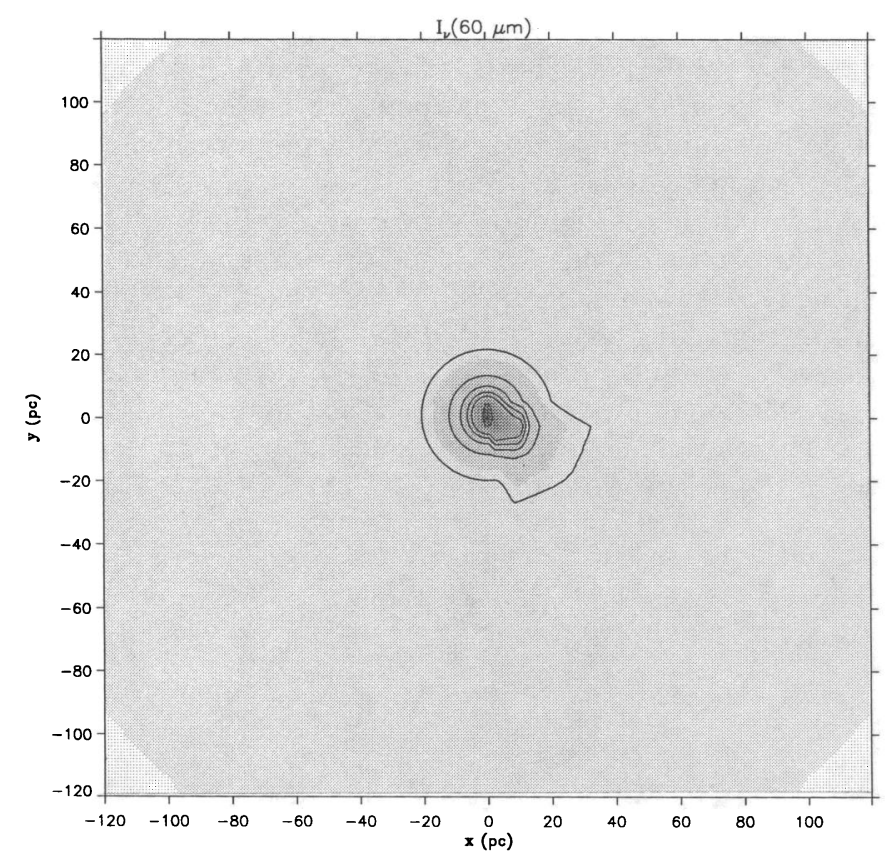

FIG. $11 c$

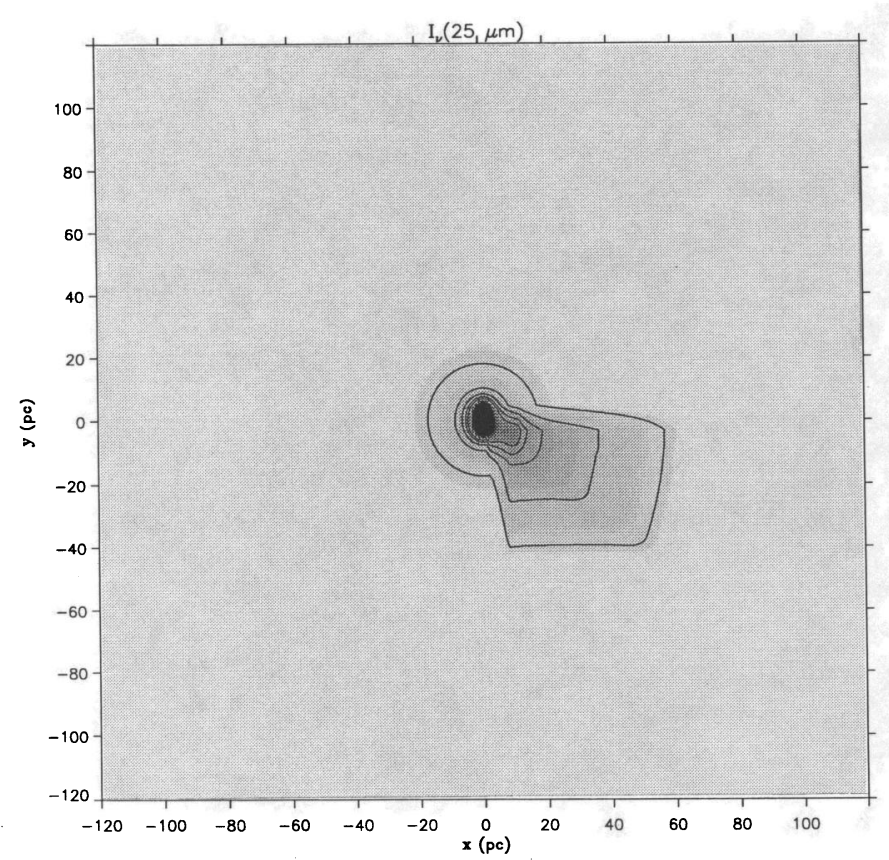

FIG. $11 b$

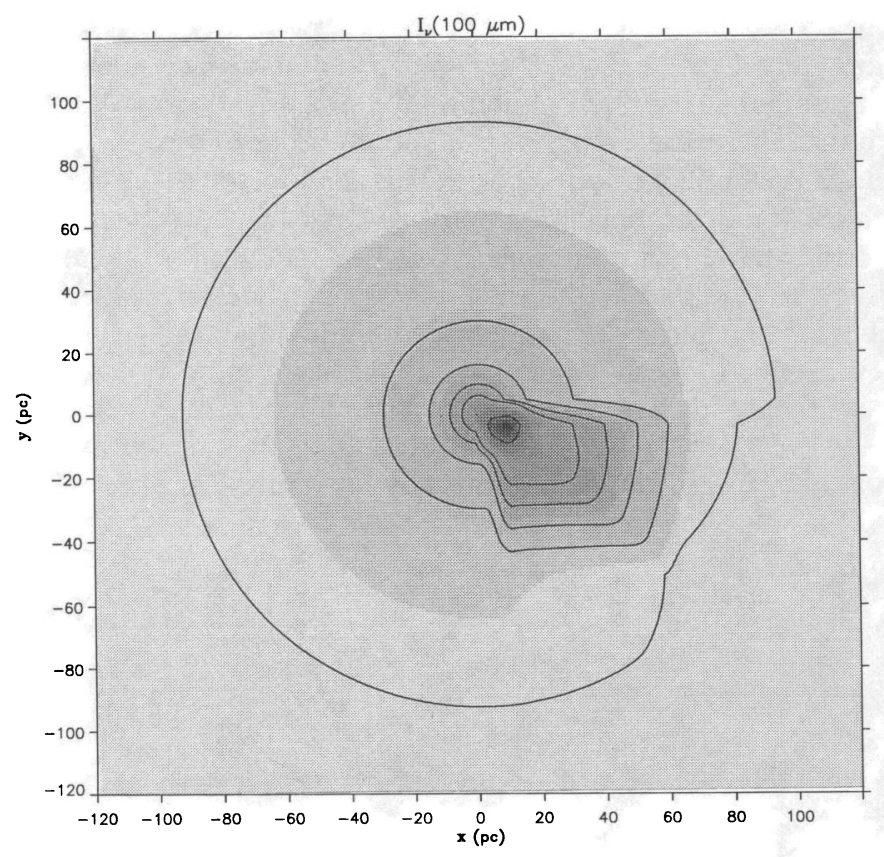

FIG. $11 d$

FIG. 11.-Cloud orientation case 2. Otherwise, analogous to Fig. 10. The warm cloud surface is seen nearly in projection against the star cluster. The cloud shadow is evident in Fig. $11 d$. 
active binaries and single stars, since the dynamo mechanism should be affected by the rotational history of stars, which is not the same for single and binary stars.

Giménez et al. (1991), studying a sample of active binaries of groups 1 and 2, found a large deviation of the color index $m_{1}$ from the expected main-sequence values which cannot be due to a metal deficiency alone. These authors showed that an important contribution to the observed $m_{1}$ deficiency may be due to some degree of stellar activity, and they found a certain correlation between $\Delta V$ (amplitude of the light variation outside eclipse, migrating wave) and the obtained value of $\delta m_{1}$. In order to check the above correlation, we plot in Figure 11 $C(\mathrm{~K})$ versus $\delta m_{1}$ for the systems belonging to groups 1 and 2 . A correlation between these two parameters is found which confirms the relation between the $m_{1}$ deficiency and the degree of activity. This result supports the idea that RS CVn systems and BY Dra stars are not metal underabundant. The $m_{1}$ deficiency is due to the fact that the chromospheric activity originates a filled-in core of metallic lines.

Finally, the $\mathrm{H} \alpha$ emission equivalent width $\left(\mathrm{EW}_{e}\right)$ does not show a clear dependence on $\mathrm{Ca}$ II $\mathrm{H}$ and $\mathrm{K}$ surface fluxes, $F(\mathrm{H}+\mathrm{K})$, since, although stars with higher values of $\mathrm{EW}_{e}$ have stronger $\mathrm{Ca}$ II $\mathrm{H}$ and $\mathrm{K}$ emission, there are several stars without filled-in absorption which also have high $F(\mathrm{H}+\mathrm{K})$ values.

According to the above arguments we ought to conclude that the enhanced activity in RS CVn systems and BY Dra stars is mainly a consequence of their fast rotation. However, there is not a straightforward relation of the chromospheric activity indicators with stellar parameters.

More observational and theoretical efforts should be devoted in order to achieve more accurate absolute parameters, and to obtain a deeper insight into the complex mechanism responsible of the underlying magnetic activity.

The INT $2.5 \mathrm{~m}$ on the island of La Palma and the $2.2 \mathrm{~m}$ telescope at Calar Alto Observatory are operated, respectively,

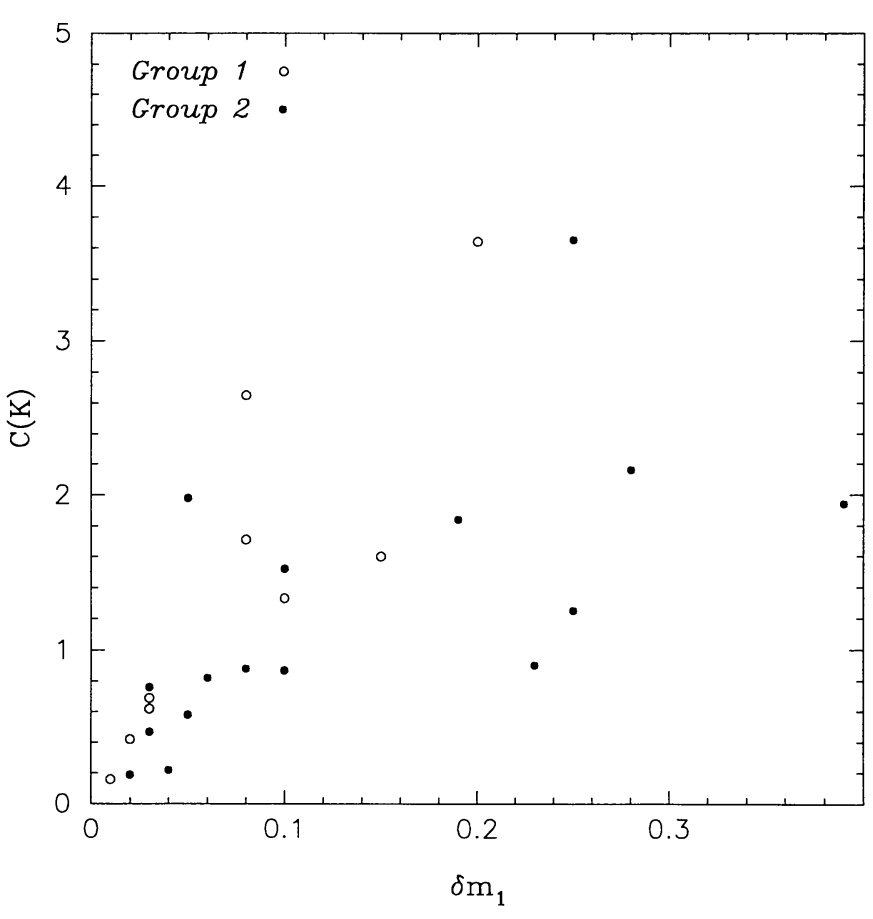

FIG. 11.-Relation between the parameter $C(\mathrm{~K})$ and the $m_{1}$ deficiency $\delta m_{1}$ for the systems belonging to groups 1 and 2 .

by the Royal Greenwich Observatory at the Spanish Observatorio del Roque de Los Muchachos (Instituto de Astrofísica de Canarias), and the Max Planck Institut für Astronomie at the Centro Astronómico Hispano-Alemán of Calar Alto (Almería, Spain). We want to express our gratitude to the staff of both observatories for their help during the observations. This work has been supported by the Universidad Complutense de Madrid and the Spanish Dirección General de Investigación Científica y Técnica (DGICYT) under grant PB91-0348.
Armentia, J. E., Fernádez-Figueroa, M. J., Cornide, M., De Castro, E., \& Fabregat, J. 1990, Active Close Binaries (Dordrecht: Kluwer), 551

Baliunas, S. L., \& Dupree, A. K. 1982, ApJ, 252, 668

Barden, S. C. 1984, AJ, 89, 683

- 1985, ApJ, 295, 162

Barnes, J. V., \& Hayes, D. S. 1984, IRS Standard Manual

Batten, A. H., Fletcher, J. M., \& Mann, P. J. 1978, Publ. Dom. Astrophys. Obs. Victoria, 15 , No. 5

Batten, A. H., Fisher, W. A., Fletcher, J. M., \& Hill, G. 1983, PASP, 95, 768

Blanco, C., Catalano, S., Marilli, E., \& Rodonò, M. 1974, A\&A, 33, 257

Bopp, B. W. 1983, in IAU Colloq. 71, Activity in Red-Dwarf Stars, ed.

P. B. Byrne, \& M. Rodonò (Dordrecht: Reidel), 363 1984, ApJS, 54, 387

Bopp, B. W., Africano, J. L., Stencel, R. E., Noah, P. V., \& Klimke, A. 1983, ApJ, 275, 691

Bopp, B. W., Dempsey, R. C., \& Maniak, S. 1988, ApJS, 68, 803

Bopp, B. W., \& Fekel, F. C. 1976, AJ, 81, 771 . 1977, AJ, 82, 490

Bopp, B. W., Fekel, F. C., Griffin, R. F., Bearvers, W. I., Gunn, J. E., \& Edwards, D. 1979, AJ, 84, 1763

\section{REFERENCES}

Bopp, B. W., Fekel, F. C., Hall, D. S., Henry, G. W., Noah, P. V., Africano, J. L., Wilkerson, M. S., \& Beavers, W. I. 1982, AJ, 87, 1035

Bopp, B. W., \& Talcott, J. C. 1978, AJ, 83, 1517

. 1980, AJ, 85, 55

Buzasi, D. L., Huenemoerder, D. P., \& Ramsey, L. W. 1991, PASP, 103, 1077

Cornide, M., Fernández-Figueroa, M. J., De Castro, E., Armentia, J. E., \& Reglero, V. 1992, AJ, 103, 1374

Cowley, A. P., \& Bildelman, W. P. 1979, PASP, 91, 83

Dermican, O. 1990, Active Close Binaries (Dordrecht: Kluwer)

De Castro, E., Fernández-Figueroa, M. J., Cornide, M., \& Armentia, J. E. 1990, Ap\&SS, 170, 99

Eker, Z. 1992, ApJS, 79, 481

Fabregat, J. 1989, Ph.D. thesis, Univ. Valencia, Spain

Fekel, F. C. 1988, AJ, 95, 215

Fekel, F. C., Moffett, T. J., \& Henry, G. W. 1986, ApJS, 60, 551

Fekel, F. C., Quigley, R., \& Africano, J. L. 1991, AJ, 94, 726

Fekel, F. C., \& Simon, T. 1985, ApJ, 90, 812

Fernández-Figueroa, M. J., Montesinos, B., De Castro, E., Rego, M., Giménez, A., \& Reglero, V. 1986a, A\&A, 169, 219 
Fernández-Figueroa, M. J., De Castro, E., Montesinos, B., Barreiro, F. J., Giménez, A., \& Reglero, V. 1986b, Adv. Space. Res., 6, 187

Fernández-Figueroa, M. J., Armentia, J. E., De Castro, E., \& Cornide, M. 1990, in IAU Colloq. 130, The Sun and Cool Stars: Activity, Magnetism, and Dynamos, ed. I. Tuominen, D. Moss, \& G. Rudiger (Berlin: Springer), 483

Garcia, M., Baliunas, S. L., Conroy, M., Johnston, M. D., Ralph, E., Roberts, W., Schwartz, D. A., \& Tonroy, J. 1980, ApJ, 240, L107

Giménez, A., Reglero, V., De Castro, E., \& Fernández-Figueroa, M. J. 1991, A\&A, 248, 563

Hall, D. S. 1976, in IAU Symp. 29, Multiple Periodic Variable Stars, ed. W. S. Fitch (Dordrecht: Reidel), 287

$\longrightarrow$. 1986, ApJ, 309, L83

Hall, D. S., Henry, G. W., \& Louth, H. 1982, ApJ, 257, L91

Hall, D. S., \& Kreimer, J. M. 1980, Acta. Astron., 30, 387

Hayes, D. S., \& Latham, D. W. 1975, ApJ, 197, 593

Hearnshaw, J. B. 1979, in IAU Colloq. 46, Changing Trends in Variable Star Research, ed. F. M. Bateson, J. Smak, \& I. H. Urch (Hamilton: Univ. Waikato), 371

Hinderer, F. 1957, Astron. Nach., 284, 1

Hoffleit, D., \& Jaschek, C. 1982, The Bright Star Catalogue, 4th ed. (New Haven: Yale Univ. Press)

Hossak, W. R. 1954, JRASC, 48, 211

Huenemoerder, D. P., \& Barden, S. C. 1986, AJ, 91, 583

Keenan, P. C., \& Pitts, R. E. 1980, ApJ, 138, 832

Levato, H. 1975, A\&AS, 19, 91

Linsky, J. L. 1984, in Cool Stars, Stellar Systems and the Sun, ed. S. Baliunas \& L. Hartmann (Heidelberg-Springer), 244

Linsky, J. L., \& Ayres, T. R. 1978, ApJ, 220, 619

Montes, D., De Castro, E., Cornide, M., \& Fernández-Figueroa, M. J. 1993, in Advances in Stellar and Solar Coronal Physics, ed. J. L. Linsky \& S. Serio (Dordrecht: Kluwer), in press
Montesinos, B. 1986, Ph.D. thesis, Univ. Complutense de Madrid, Spain Montesinos, B., Giménez, A., \& Fernández-Figueroa, M. J. 1988, MNRAS, 232, 361

Oke, J. B., \& Gunn, J. E. 1983, ApJ, 266, 713

Pasquini, L., Pallavicini, R., \& Pakull, M. 1988, A\&A, 191, 253

Popper, D. M. 1988, AJ, 95, 1242

1990, AJ, 100, 247

Reglero, V., Fernández-Figueroa, M. J., Giménez, A., De Castro, E., Fabregat, J., Cornide, M., \& Armentia, J. E. 1991, A\&AS, 88, 545

Rodonò, M. 1992, in Evolutionary Processes in Interacting Binary Stars, ed. Y. Kondo et al. (Dordrecht: Kluwer), 71

Schmidt-Kaler, T. 1982, in Landolt-Börnstein, Vol. 2b, ed. K. Schaifers \& H. H. Voig (Heidelberg: Springer), 453

Smith, S. E., \& Bopp, B. W. 1982, Ap. Lett., 22, 127

Stencel, R. E. 1978, ApJ, 223, L37

Strassmeier, K. G. 1990, ApJ, 348, 682

Strassmeier, K. G., et al. 1988a, A\&A, 192, 135

Strassmeier, K. G., \& Fekel, F. C. 1990, A\&A, 320, 389

Strassmeier, K. G., Fekel, F. C., Bopp, B. W., Dempsey, R. C., \& Henry, G. W. 1990, ApJS, 72, 191

Strassmeier, K. G., Hall, D. S., Boyd, L. J., \& Genet, R. M. 1989, ApJS, 69, 141

Strassmeier, K. G., Hall, D. S., Zeilik, M., Nelson, E., Eker, Z., \& Fekel, F. C. 1988b, A\&AS, 72, 291 (CABS)

Weiler, E. J. 1978, MNRAS, 182, 77

Wilson, O. C. 1963, ApJ, 138, 832 . 1976, ApJ, 205, 823

Xuefu, L., \& Huisong, T. 1984, Inf. Bull. Var. Stars, 2606 1986, Chinese A\&A, 10, 221

Young, A., \& Koniges, A. 1977, ApJ, 211, 836 